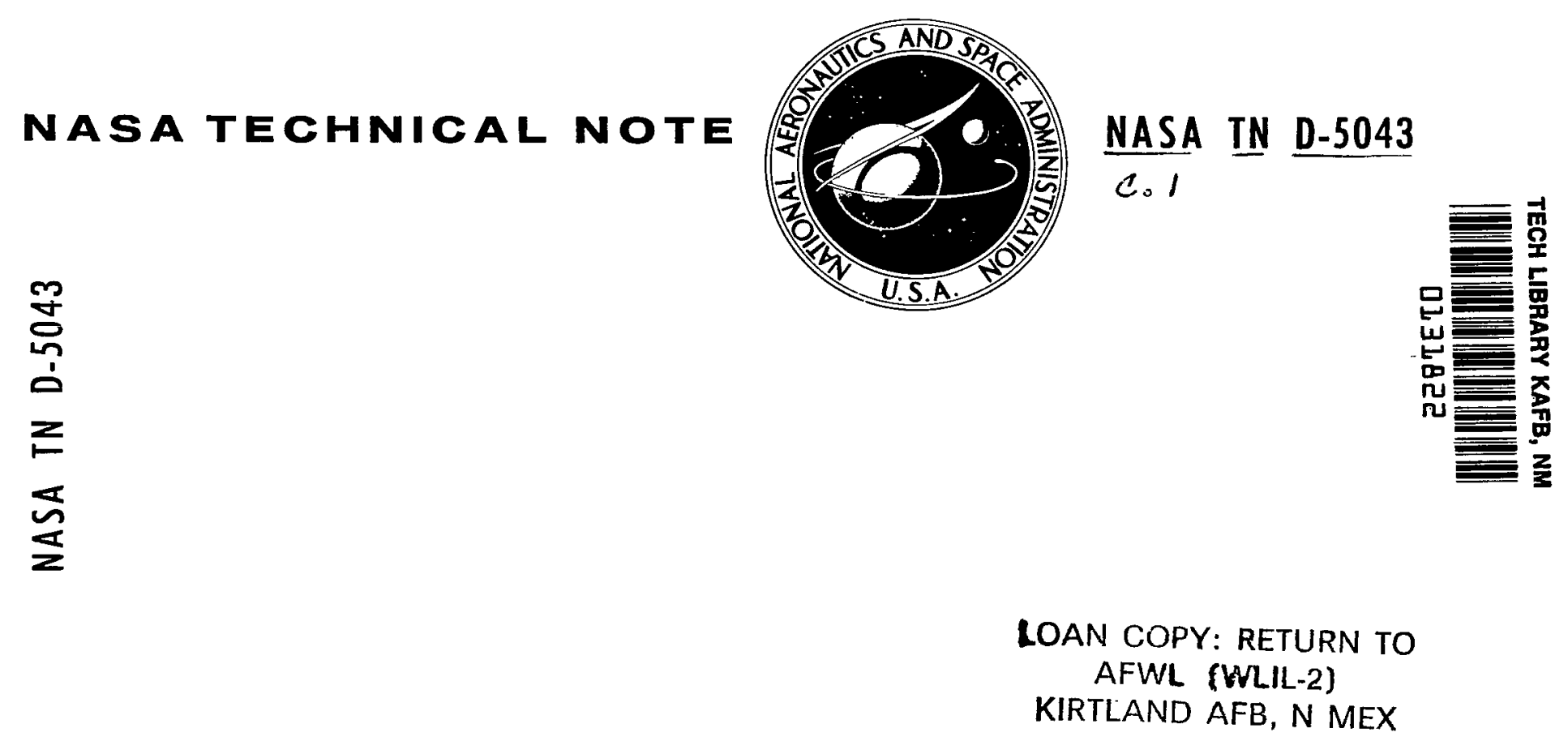

\title{
A SEMI-ANALYTIC THEORY \\ FOR THE MOTION \\ OF A LUNAR SATELLITE
}

by

Giorgio E. O. Giacaglia

Yale University

and

James P. Murphy and Theodore L. Felsentreger

Goddard Space Flight Center

NATIONAL AERONAUTICS AND SPACE ADMINISTRATION - WASHINGTON, D. C. • JUNE 1969 
A SEMI-ANALYTIC THEORY FOR THE MOTION OF A LUNAR SATELLITE

By Giorgio E. O. Giacaglia

Yale University New Haven, Conn.

and

James P. Murphy and Theodore L. Felsentreger

Goddard Space Flight Center

Greenbelt, Md. 


\begin{abstract}
A semi-analytical solution to the problem of the motion of a satellite of the moon is presented. The theory is developed to third order, where first order is $10^{-2}$. Perturbative effects which are considered include those due to the attraction of the moon, earth, and sun, the non-sphericity of the moon's gravitational field, the oblateness of the earth, coupling of lower-order terms, solar radiation pressure, and physical libration. Short-period terms and those with the period of the moon's longitude are produced by means of von Zeipel's method; it is proposed to obtain the secular perturbations, and those depending only on the argument of perilune, by numerical integration of the equations of motion.
\end{abstract}




\section{CONTENTS}

Abstract. .............................. ii

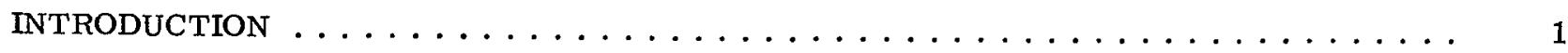

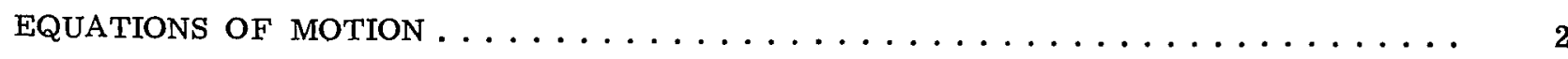

OBLATENESS TERMS $\ldots \ldots \ldots \ldots \ldots \ldots \ldots \ldots \ldots \ldots \ldots \ldots \ldots \ldots \ldots \ldots$

CANONICAL EQUATIONS AND GRAVITATIONAL TERMS . . . . . . . . . . . 7

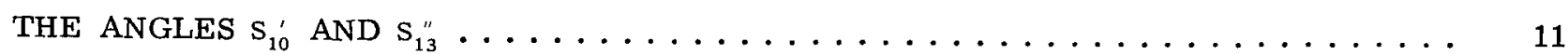

THE MAIN PROBLEM ............................ 12

DEVELOPMENT OF THE DISTURBING FUNCTION . . . . . . . . . . . . . . . 14

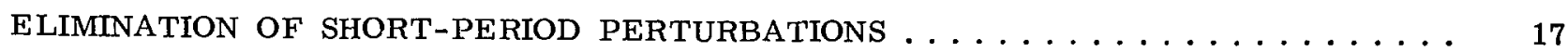

THE LONG-PERIOD TERMS; ELIMINATION OF $h^{\prime} \ldots \ldots \ldots \ldots \ldots \ldots \ldots \ldots$

THE SECOND-ORDER LONG-PERIOD TERMS AND

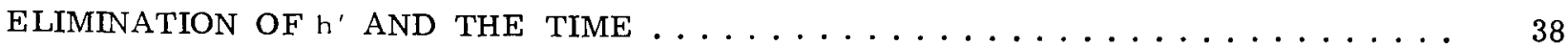

THIRD-ORDER TERMS GENERATED BY COUPLING OF SECOND-ORDER TERMS . . . 41

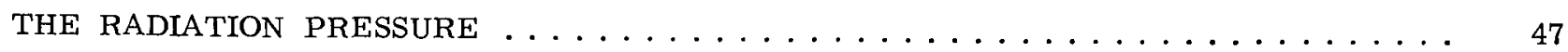

THE SECOND LEGENDRE POLYNOMIAL FOR THE

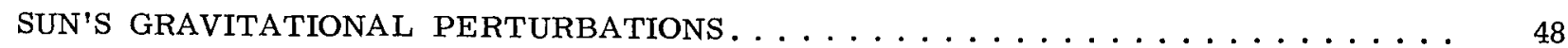

THE THIRD LEGENDRE POLYNOMIAL FOR THE

EARTH'S GRAVITATIONAL PERTURBATIONS .................. 50

THE ECCENTRICITY OF THE MOON'S ORBIT .................. 52

THE INCLINATION OF THE MOON'S ORBIT TO ITS EQUATOR............ 53

THE NON-SPHERICITY OF THE POTENTIAL FIELD OF THE EARTH . . . . . . . . 54

PHYSICAL LIBRATION, AND THE PRECESSION OF THE LUNAR EQUATOR. . . . . . 57

COMPLETE "SECULAR" THIRD-ORDER HAMILTONIAN . . . . . . . . . . . . . 59

THE DETERMINING FUNCTION FOR LONG-PERIOD TERMS . . . . . . . . . . 59

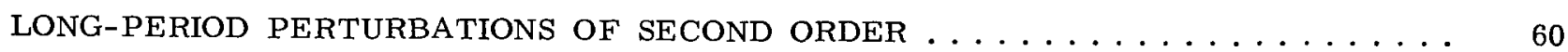

SECULAR PERTURBATIONS AND PERTURBATIONS DEPENDING STRICTLY ON g" . . 81

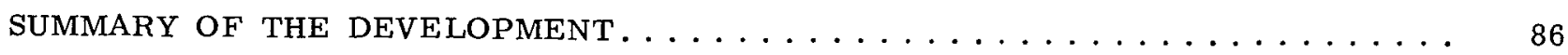

POSITION AND VELOCITY e $\neq 0 ; \mathrm{I} \neq 0^{\circ}, 180^{\circ} \ldots \ldots \ldots \ldots \ldots$

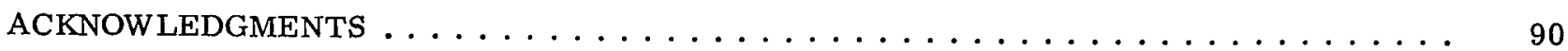

References ............................... 90

Bibliography ................................ 91 


\title{
A SEMI-ANALYTIC THEORY FOR THE MOTION OF A LUNAR SATELLITE
}

\author{
by \\ Giorgio E. O. Giacaglia* \\ Yale University \\ and \\ James P. Murphy and Theodore L. Felsentreger \\ Goddard Space Flight Center
}

\section{INTRODUCTION}

The motion of a satellite of the moon, or lunar orbiter, is analyzed. The solution is developed in powers of $10^{-2}$-i.e., first order is $10^{-2}$, second order is $10^{-4}$, etc. The Hamiltonian for the "main problem" consists of zero-, first-, and second-order quantities. The higher-order Hamiltonian is of third order and consists of two parts, the first containing terms generated by coupling of lower-order terms and the second consisting of terms added by considering further perturbing forces, such as solar radiation pressure, physical libration, non-sphericity of the earth's potential field, the attraction of the sun, etc. Additional terms are produced by considering also the eccentricity and inclination of the moon's orbit.

In order to retain the relative orders of the disturbing forces, it is necessary to restrict the semi-major axis of the orbiter to about four moon radii or less. Furthermore, certain restrictions must be made on the eccentricity and inclination in order not to invalidate the solution. Therefore, the following assumptions on the semi-major axis, eccentricity, and inclination are made:

$$
\begin{aligned}
a & \leq 4 \text { moon radii } \\
.01<e & <.75 \\
\sin \mathrm{I} & >.01 .
\end{aligned}
$$

The small parameter of first order is $\mathrm{n}_{\mathbb{C}}^{*}$, which is the mean motion of the moon's mean longitude. Recent determinations of the spherical harmonics of the moon (see References 1,2 , and 3 ) indicate that the second degree zonal and sectorial harmonic coefficients, together with the third,

*Now at the University of São Paulo, Brazil. 
fourth, and fifth degree zonal harmonic coefficients, are all of about the same order of magnitudenamely, $10^{-4}$. Therefore, the small parameters of second order are $J_{2}, J_{22}, J_{3}, J_{4}, J_{5}$, and $\left(n_{\mathbb{C}} / n\right)^{2}$, where $J_{2}$ and $J_{22}$ define the principal part of the oblateness of the moon and $\left(n_{\mathbb{C}} / n\right)^{2}$ is the square of the ratio of the mean motion of the moon to that of the orbiter. The quantities $\mathrm{J}_{3}, \mathrm{~J}_{4}$, and $\mathrm{J}_{5}$ are higher degree zonal harmonic parameters of the moon. The small parameters of third order are $j_{2}\left(n_{\mathbb{C}} / n\right)^{2},\left(n_{\oplus} / n\right)^{2},\left(n_{\mathbb{C}} / n\right)^{2} \sin \left(i_{\mathbb{C}} / 2\right),\left(n_{\mathbb{C}} / n\right)^{2} e_{\mathbb{C}}, \sigma,\left(n_{\mathbb{C}} / n\right)^{3}$, and an ${ }_{\odot} / n$. Here, the parameter $j_{2}$ is the principal part of the oblateness of the earth. The quantity $\left(n_{\oplus} / n\right)^{2}$ is the square of the ratio of the mean motion of the earth to that of the orbiter. The fact that the moon's orbital plane is inclined to its equator and the fact that the moon's orbit about the earth is elliptical give rise to the two small parameters of third order $\left(n_{\mathbb{C}} / n\right)^{2} \sin \left(i_{\mathbb{C}} / 2\right)$ and $\left(n_{\mathbb{C}} / n\right)^{2} e_{\mathscr{C}}$, respectively. The radiation pressure gives rise to the third order parameter $\sigma$, and $\left(n_{\mathbb{C}} / n\right)^{3}$ is the cube of the ratio of the mean motion of the moon to that of the orbiter. Finally, $\alpha_{\infty} / n$ is the correction due to physical libration.

The longest meridian of the moon contains the line joining the centers of mass of the earth and the moon. The right-handed, rotating, selenocentric coordinate systems adopted for this problem will then be as follows: The $z$-axis is the rotational axis of the moon, and the $x y$-plane is the moon's equatorial plane. The $\mathrm{x}$-axis passes through the moon's longest meridian, and is assumed to rotate with the motion $\mathrm{n}_{\mathbb{C}}^{*}$.

For a semi-major axis of 4 moon radii,

$$
\begin{aligned}
& \left(\frac{n_{\mathscr{C}}}{n}\right)^{2} \simeq 4.8 \times 10^{-4} \\
& \left(\frac{n_{\oplus}}{n}\right)^{2} \simeq 2.7 \times 10^{-6}
\end{aligned}
$$

so that the oblateness terms in the lunar potential and the earth perturbations are both of about the same order. The largest oblateness term of the earth is then of the order of $10^{-6}$. The perturbation of the sun is of third order.

Another perturbation to be considered is the effective radiation pressure of the sun, whose strength is about $1 \times 10^{-4} \mathrm{dyne} / \mathrm{cm}^{2}$. If the area-mass ratio of the orbiter is $1.5 \times 10^{-1} \mathrm{~cm}^{2} / \mathrm{g}$, then the disturbing acceleration due to radiation pressure is also of third order.

\section{EQUATIONS OF MOTION}

The first step is to determine the equations of motion for the gravitational fields of the moon as a primary and the earth and sun as perturbations.

The following notation will be used:

$$
\begin{aligned}
& \text { subscript } 0: \text { moon } \\
& \text { subscript } 1: \text { orbiter } \\
& \text { subscript } 2: \text { earth } \\
& \text { subscript } 3: \text { sun. }
\end{aligned}
$$


In an inertial system, the equations of motion are

$$
\mathrm{m}_{\mathrm{j}} \ddot{\rho}_{\mathrm{j}}=\operatorname{grad}_{\rho_{\mathrm{j}}} \mathrm{U}(\mathrm{j}=0,1,2,3)
$$

where

$$
\mathrm{U}=\mathrm{k}^{2}\left(\frac{\mathrm{m}_{0} \mathrm{~m}_{1}}{\mathrm{r}_{01}}+\frac{\mathrm{m}_{0} \mathrm{~m}_{2}}{\mathrm{r}_{02}}+\frac{\mathrm{m}_{0} \mathrm{~m}_{3}}{\mathrm{r}_{03}}+\frac{\mathrm{m}_{1} \mathrm{~m}_{2}}{\mathrm{r}_{12}}+\frac{\mathrm{m}_{1} \mathrm{~m}_{3}}{\mathrm{r}_{13}}+\frac{\mathrm{m}_{2} \mathrm{~m}_{3}}{\mathrm{r}_{23}}\right)
$$

and $\rho_{j}$ is the radius vector of any one of the four bodies. If $\xi_{j}, \eta_{j}, \zeta_{j}$ are the rectangular inertial coordinates of one of the bodies, then the equations of motion can also be written

$$
m_{j} \ddot{\xi}_{j}=\frac{\partial U}{\partial \ddot{\xi}_{j}}(j=0,1,2,3) .
$$

Similar expressions hold for $\eta_{\mathrm{j}}$ and $\zeta_{\mathrm{j}}$.

It is now convenient to refer the orbiter to a moon-centered system, the moon to an earthcentered system, and the sun to a system whose origin is at the center of mass of the earth-moon system, designated by the subscript G. Therefore,

$$
\begin{aligned}
& x_{1}=\xi_{1}-\xi_{0}, \\
& x_{0}-\xi_{0}-\xi_{2} .
\end{aligned}
$$

and

$$
\mathrm{x}_{3}=\xi_{3}-\xi_{\mathrm{G}}=\xi_{3}-\frac{\mathrm{m}_{0} \xi_{0}+\mathrm{m}_{2} \xi_{2}}{\mathrm{~m}_{0}+\mathrm{m}_{2}} .
$$

Similar expressions hold for $y_{j}$ and $z_{j}$.

The equations of motion must be transformed accordingly. The partials in Equation 2 are then computed with respect to the new variables by making use of

$$
\frac{\partial \mathrm{U}}{\partial \xi_{\mathrm{k}}}=\sum_{\mathrm{j}=0}^{3} \frac{\partial \mathrm{U}}{\partial \mathbf{x}_{\mathrm{j}}} \frac{\partial \mathbf{x}_{\mathrm{j}}}{\partial \xi_{\mathrm{k}}}(\mathrm{k}=0,1,2,3)
$$




\section{It follows that}

$$
\begin{aligned}
& \frac{\partial U}{\partial \xi_{0}}=\frac{\partial U}{\partial x_{0}}-\frac{\partial U}{\partial x_{1}}-\frac{m_{0}}{m_{0}+m_{2}} \frac{\partial U}{\partial x_{3}}, \\
& \frac{\partial U}{\partial \xi_{1}}=\frac{\partial U}{\partial x_{1}}, \\
& \frac{\partial U}{\partial \xi_{2}}=-\frac{\partial U}{\partial x_{0}}-\frac{m_{2}}{m_{0}+m_{2}} \frac{\partial U}{\partial x_{3}},
\end{aligned}
$$

and

$$
\frac{\partial U}{\partial \xi_{3}}=\frac{\partial U}{\partial x_{3}}
$$

Since

$$
\ddot{x}_{1}=\ddot{\xi}_{1}-\ddot{\xi}_{0},
$$

it follows that

$$
\ddot{x}_{1}=\left(\frac{1}{m_{1}}+\frac{1}{m_{0}}\right) \frac{\partial U}{\partial x_{1}}-\frac{1}{m_{0}} \frac{\partial U}{\partial x_{0}}+\frac{1}{m_{0}+m_{2}} \frac{\partial U}{\partial x_{3}} .
$$

The force function $U$ must now be expressed in terms of the new coordinates. We have

$$
\begin{aligned}
r_{01}^{2} & =\left(\xi_{1}-\xi_{0}\right)^{2}+\left(\eta_{1}-\eta_{0}\right)^{2}+\left(\zeta_{1}-\zeta_{0}\right)^{2}=x_{1}^{2}+y_{1}^{2}+z_{1}^{2}=r_{1}^{2}, \\
r_{02}^{2} & =\left(\xi_{0}-\xi_{2}\right)^{2}+\cdots=x_{0}^{2}+\cdots=r_{0}^{2}, \\
r_{03}^{2} & =\left(\xi_{3}-\xi_{0}\right)^{2}+\cdots=\left(x_{3}-\frac{m_{2}}{m_{0}+m_{2}} x_{0}\right)^{2}+\cdots \\
& =x_{3}^{2}+y_{3}^{2}+z_{3}^{2}+\left(\frac{m_{2}}{m_{0}+m_{2}}\right)^{2}\left(x_{0}^{2}+y_{0}^{2}+z_{0}^{2}\right)-\frac{2 m_{2}}{m_{0}+m_{2}}\left(x_{0} x_{3}+y_{0} y_{3}+z_{0} z_{3}\right) \\
& =r_{3}^{2}+\left(\frac{m_{2}}{m_{0}+m_{2}}\right)^{2} r_{0}^{2}-\frac{2 m_{2}}{m_{0}+m_{2}} \vec{r}_{0} \cdot \vec{r}_{3} \cdot \\
r_{12}^{2} & =\left(\xi_{1}-\xi_{2}\right)^{2}+\cdots=\left(x_{1}+x_{0}\right)^{2}+\cdots=r_{1}^{2}+r_{0}^{2}+2 \vec{r}_{1} \cdot \vec{r}_{0},
\end{aligned}
$$




$$
\begin{aligned}
r_{13}^{2} & =\left(\xi_{1}-\xi_{3}\right)^{2}+\cdots=\left[\left(x_{1}-x_{3}\right)+\frac{m_{2}}{m_{0}+m_{2}} x_{0}\right]^{2}+\cdots \\
& =r_{1}^{2}+r_{3}^{2}-2 \vec{r}_{1} \cdot \vec{r}_{3}+\left(\frac{m_{2}}{m_{0}+m_{2}}\right)^{2} r_{0}^{2}+\frac{2 m_{2}}{m_{0}+m_{2}}\left(\vec{r}_{0} \cdot \vec{r}_{1}-\vec{r}_{0} \cdot \vec{r}_{3}\right) .
\end{aligned}
$$

and

$$
\begin{aligned}
r_{23}^{2} & =\left(\xi_{2}-\xi_{3}\right)^{2}+\cdots=\left(-x_{3}-\frac{m_{0}}{m_{0}+m_{2}} x_{0}\right)^{2}+\cdots \\
& =r_{3}^{2}+\left(\frac{m_{0}}{m_{0}+m_{2}}\right)^{2} r_{0}^{2}+\frac{2 m_{0}}{m_{0}+m_{2}} \vec{r}_{0} \cdot \vec{r}_{3} .
\end{aligned}
$$

Since $U$ contains the inverses of these radii, note that

$$
\begin{aligned}
& \frac{1}{r_{01}}=\frac{1}{r_{1}}, \\
& \frac{1}{r_{02}}=\frac{1}{r_{0}} \\
& \frac{1}{r_{03}}=\frac{1}{r_{3}}\left[1+\left(\frac{m_{2}}{m_{0}+m_{2}}\right)^{2}\left(\frac{r_{0}}{r_{3}}\right)^{2}-\frac{2 m_{2}}{m_{0}+m_{2}} \frac{r_{0}}{r_{3}} \cos S_{03}\right]^{-1 / 2}, \\
& \frac{1}{r_{12}}=\frac{1}{r_{0}}\left[1+\left(\frac{r_{1}}{r_{0}}\right)^{2}+2 \frac{r_{1}}{r_{0}} \cos S_{10}\right]^{-1 / 2}, \\
& \frac{1}{r_{13}}-\frac{1}{r_{3}}\left[1+\left(\frac{r_{1}}{r_{3}}\right)^{2}-2 \frac{r_{1}}{r_{3}} \cos S_{13}+\left(\frac{m_{2}}{m_{0}+m_{2}}\right)^{2}\left(\frac{r_{0}}{r_{3}}\right)^{2}+\frac{2 m_{2}}{m_{0}+m_{2}}\left(\frac{r_{0} r_{1}}{r_{3}^{2}} \cos S_{10}-\frac{r_{0}}{r_{3}} \cos S_{03}\right)\right]^{-1 / 2},
\end{aligned}
$$

and

$$
\frac{1}{r_{23}}=\frac{1}{r_{3}}\left[1+\left(\frac{m_{0}}{m_{0}+m_{2}}\right)^{2}\left(\frac{r_{0}}{r_{3}}\right)^{2}+\frac{2 m_{0}}{m_{0}+m_{2}} \frac{r_{0}}{r_{3}} \cos S_{03}\right]^{-1 / 2} .
$$

The angles used are shown in Figure 1. The angle $S_{0_{3}}$ may be expressed in terms of $S_{03}^{\prime}$, as follows: Since

$$
\cos S_{03}^{\prime}=\frac{\vec{r}_{0} \cdot \vec{r}_{3}^{\prime}}{r_{0} r_{3}^{\prime}},
$$

and

$$
\frac{m_{0}}{m_{0}+m_{2}} \vec{r}_{0}+\vec{r}_{3}=\vec{r}_{3}^{\prime}
$$




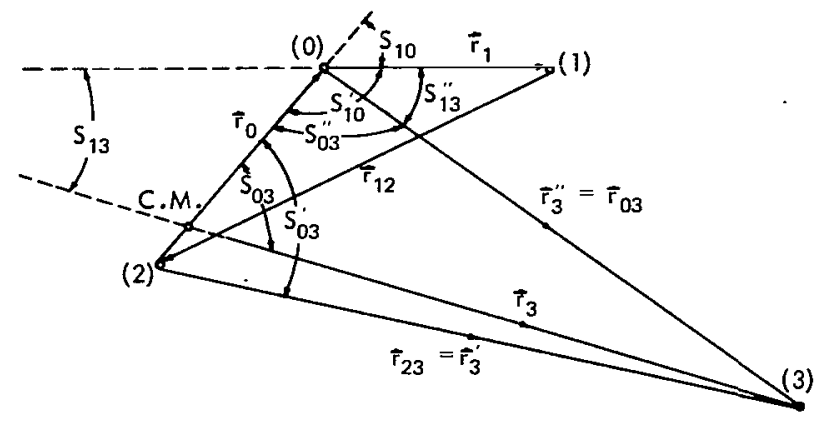

then

$$
\begin{aligned}
\cos S_{03}^{\prime} & =\frac{\frac{m_{0}}{m_{0}+m_{2}} r_{0}^{2}+r_{0} r_{3} \cos S_{03}}{r_{0} r_{3}^{\prime}} \\
& =\frac{r_{0}}{r_{3}^{\prime}} \cdot \frac{m_{0}}{m_{0}+m_{2}}+\frac{r_{3}}{r_{3}{ }^{\prime}} \cos S_{03} .
\end{aligned}
$$

Figure 1-Relative positions of the moon, orbiter, earth, and sun.

Finally,

$$
\cos S_{03}=\frac{r_{3}^{\prime}}{r_{3}}\left(\cos S_{03}^{\prime}-\frac{r_{0}}{r_{3}^{\prime}} \frac{m_{0}}{m_{0}+m_{2}}\right) .
$$

Certainly, to third order, $r_{3}^{\prime} \simeq r_{3}$, so that

$$
\cos S_{03}=\cos S_{03}^{\prime}-\frac{r_{0}}{r_{3}} \frac{m_{0}}{m_{0}+m_{2}} .
$$

On the other hand, $S_{10}$ may be replaced by $180^{\circ}-S_{10}^{\prime}$ where $S_{10}^{\prime}$ is the selenocentric elongation between the earth and the orbiter.

\section{OBLATENESS TERMS}

If the plane of reference is the lunar equatorial plane, then the disturbing force per unit mass may be written as

$$
U_{O B L}=\frac{k^{2} m_{0}}{r_{1}} \sum_{n=1}^{\infty} \sum_{m=0}^{n}\left(\frac{R_{\mathscr{C}}}{r_{1}}\right)^{n} J_{n m} P_{n m}(\sin \beta) \cos m\left(\lambda-\lambda_{n m}\right) .
$$

The only terms to be included are $\mathrm{J}_{22}, \mathrm{~J}_{20}=-\mathrm{J}_{2}, \mathrm{~J}_{30}=-\mathrm{J}_{3}, \mathrm{~J}_{40}=-\mathrm{J}_{4}$, and $\mathrm{J}_{50}=-\mathrm{J}_{5}$. Therefore,

$$
\mathrm{U}_{\mathrm{OBL}}=-\frac{\mu_{0}}{\mathrm{r}_{1}}\left[\sum_{\mathrm{n}=2}^{5}\left(\frac{\mathrm{R}_{\mathbb{C}}}{\mathrm{r}_{1}}\right)^{\mathrm{n}} \mathrm{J}_{\mathrm{n}} \mathrm{P}_{\mathrm{n}}(\sin \xi)-\left(\frac{\mathrm{R}_{\mathbb{C}}}{\mathrm{r}_{1}}\right)^{2} \mathrm{~J}_{22} \mathrm{P}_{22}(\sin \beta) \cos 2\left(\lambda^{\prime}-\lambda_{22}\right)\right] .
$$

The angle $\beta$ is the latitude of the orbiter with respect to the equator of the moon, $\lambda^{\prime}$ its longitude reckoned from any fixed direction, and $\lambda_{22}$ the longitude of the moon's longest meridian from the 
same fixed direction. Note that $\lambda^{\prime}$ and $\beta$ can be expressed in terms of the coordinates $x_{1}, y_{1}, z_{1}$ of the orbiter. However, $\lambda_{22}$ will contain the time explicitly, since

$$
\lambda_{22}=\lambda_{22}(0)+\gamma_{\mathbb{C}} t,
$$

where $\gamma_{\mathbb{C}}$ is the frequency of rotation of the moon around its axis. Further, if we neglect the physical libration of the moon, the longest meridian is always pointing toward the earth and $\gamma_{\mathbb{C}} \simeq n_{\mathbb{C}}^{*}$.

\section{CANONICAL EQUATIONS AND GRAVITATIONAL TERMS}

Let us choose as canonical variables the Delaunay set

$$
\begin{aligned}
& \text { L } \quad \sqrt{\mu_{0} a^{2}}, \quad l=\text { mean anomaly, } \\
& \text { G L } \sqrt{1-c^{2}}, \quad a=\text { argument of pericenter }, \\
& \text { H G cos I } \quad S:=\text { longitude of ascending node, }
\end{aligned}
$$

where a, e, and I are the semi-major axis, eccentricity, and inclination, respectively, and where $\mu_{0}=\mathrm{k}^{2} \mathrm{~m}_{0}$, in which $\mathrm{k}$ is the Gaussian constant and $\mathrm{m}_{0}$ is the mass of the moon. Then the equations of motion become

$$
\begin{aligned}
& \dot{\mathrm{L}} \quad \frac{\partial \widetilde{\mathrm{F}}}{\partial l}, \quad \dot{\mathrm{G}}=\frac{\partial \widetilde{\mathrm{F}}}{\lambda_{\mu}}, \quad \dot{\mathrm{H}}-\frac{\partial \widetilde{\mathrm{F}}}{\partial \Omega} \text {. } \\
& i=-\frac{\partial \widetilde{F}}{\partial \mathrm{L}}, \quad \therefore-\frac{\partial \tilde{\mathrm{F}}}{\partial \mathrm{G}}, \quad \dot{S}-\frac{\partial \widetilde{\mathrm{F}}}{\partial \mathrm{H}},
\end{aligned}
$$

where

$$
\widetilde{F}=\frac{\mu_{0}^{2}}{2 L^{2}}+U_{G R A V}+U_{O B L}
$$

In Equation 11, $\mathrm{U}_{\mathrm{GRAv}}$. is a function which has to satisfy the condition

$$
\ddot{x}_{1}=\frac{\partial}{\partial x_{1}}\left(U_{\text {GRAV }}+\frac{\mu_{0}}{r_{1}}\right) \text {. }
$$


The force function $\mathrm{U}$ depends on the new variables $\times$ through Equations 6 . Then,

$$
\begin{aligned}
& \frac{\partial U}{\partial x_{0}}= k^{2}\left[-m_{0} m_{2} \frac{x_{0}}{r_{02}^{3}}-m_{0} m_{3} \frac{x_{3}-\frac{m_{2}}{m_{0}+m_{2}} x_{0}}{r_{03}^{3}}\left(-\frac{m_{2}}{m_{0}+m_{2}}\right)-m_{1} m_{2} \frac{x_{1}+x_{0}}{r_{12}^{3}}\right. \\
&-\left.m_{1} m_{3} \frac{\left.x_{1}-x_{3}+\frac{m_{2}}{m_{0}+m_{2}} x_{0}\left(\frac{m_{2}}{m_{0}+m_{2}}\right)-m_{2} m_{3} \frac{-x_{3}-\frac{m_{0}}{m_{0}+m_{2}} x_{0}}{r_{23}^{3}}\left(-\frac{m_{0}}{m_{0}+m_{2}}\right)\right]}{r_{13}^{3}}\right] \\
&= \frac{\partial}{\partial x_{1}}\left[-k^{2} m_{0} m_{2} \frac{\vec{r}_{0} \cdot \vec{r}_{1}}{r_{02}^{3}}+k^{2} \frac{m_{0} m_{2} m_{3}}{m_{0}+m_{2}} \frac{\vec{r}_{3} \cdot \vec{r}_{1}}{r_{03}^{3}}-k^{2} \frac{m_{0} m_{2}^{2} m_{3}}{\left(m_{0}+m_{2}\right)^{2}} \frac{\vec{r}_{0} \cdot \vec{r}_{1}}{r_{03}^{3}}+k^{2} m_{1} m_{2} \frac{1}{r_{12}}\right. \\
&\left.+k^{2} \frac{m_{1} m_{2} m_{3}}{m_{0}+m_{2}} \frac{1}{r_{13}}-k^{2} \frac{m_{0} m_{2} m_{3}}{m_{0}+m_{2}} \frac{\vec{r}_{3} \cdot \vec{r}_{1}}{r_{23}^{3}}-k^{2} \frac{m_{0}^{2} m_{2} m_{3}}{\left(m_{0}+m_{2}\right)^{2}} \frac{\vec{r}_{0} \cdot \vec{r}_{1}}{r_{23}^{3}}\right]
\end{aligned}
$$

and

$$
\begin{aligned}
& \frac{\partial U}{\partial x_{3}}=k^{2}\left(-m_{0} m_{3} \frac{x_{3}-\frac{m_{2}}{m_{0}+m_{2}} x_{0}}{r_{03}^{3}}+m_{1} m_{3} \frac{x_{1}-x_{3}+\frac{m_{2}}{m_{0}+m_{2}}}{r_{13}^{3}}+m_{2} m_{3} \frac{-x_{3}-\frac{m_{0}}{m_{0}+m_{2}} x_{0}}{r_{23}^{3}}\right) \\
& =\frac{\partial}{\partial x_{1}}\left(-k^{2} m_{0} m_{3} \frac{\vec{r}_{3} \cdot \vec{r}_{1}}{r_{03}^{3}}+k^{2} \frac{m_{0} m_{2} m_{3}}{m_{0}+m_{2}} \frac{\vec{r}_{0} \cdot \vec{r}_{1}}{r_{03}^{3}}\right. \\
& \left.-k^{2} m_{1} m_{3} \frac{1}{r_{13}}-k^{2} m_{2} m_{3} \frac{\vec{r}_{3} \cdot \vec{r}_{1}}{r_{23}^{3}}-k^{2} \frac{m_{0} m_{2} m_{3}}{m_{0}+m_{2}} \frac{\vec{r}_{0} \cdot \vec{r}_{1}}{r_{23}^{3}}\right) \text {. }
\end{aligned}
$$

Then, substitution of Equations 13 and 14 into Equation 5 yields

$$
\begin{aligned}
\ddot{x}_{1}= & \frac{m_{1}+m_{0}}{m_{1} m_{0}} \frac{\partial}{\partial x_{1}}\left(k^{2} \frac{m_{0} m_{1}}{r_{01}}+k^{2} m_{1} m_{2} \frac{1}{r_{12}}+k^{2} m_{1} m_{3} \frac{1}{r_{13}}\right) \\
& -\frac{1}{m_{0}} \frac{\partial}{\partial x_{1}}\left(-k^{2} m_{0} m_{2} \frac{\vec{r}_{0} \cdot \vec{r}_{1}}{r_{02}^{3}}+k^{2} \frac{m_{0} m_{2} m_{3}}{m_{0}+m_{2}} \frac{\vec{r}_{3} \cdot \vec{r}_{1}}{r_{03}^{3}}-k^{2} \frac{m_{0} m_{2}^{2} m_{3}}{\left(m_{0}+m_{2}\right)^{2}} \frac{\vec{r}_{0} \cdot \vec{r}_{1}}{r_{03}^{3}}+k^{2} m_{1} m_{2} \frac{1}{r_{12}}\right.
\end{aligned}
$$




$$
\begin{aligned}
& \left.+k^{2} \frac{m_{1} m_{2} m_{3}}{m_{0}+m_{2}} \frac{1}{r_{13}}-k^{2} \frac{m_{0} m_{2} m_{3}}{m_{0}+m_{2}} \frac{\vec{r}_{3} \cdot \vec{r}_{1}}{r_{23}^{3}}-k^{2} \frac{m_{0}^{2} m_{2} m_{3}}{\left(m_{0}+m_{2}\right)^{2}} \frac{\vec{r}_{0} \cdot \vec{r}_{1}}{r_{23}^{3}}\right) \\
& +\frac{1}{m_{0}+m_{2}} \frac{\partial}{\partial x_{1}}\left[-k^{2} m_{0} m_{3} \frac{\vec{r}_{3} \cdot \vec{r}_{1}}{r_{03}^{3}}+k^{2} \frac{m_{0} m_{2} m_{3}}{\left(m_{0}+m_{2}\right)} \frac{\vec{r}_{0} \cdot \vec{r}_{1}}{r_{03}^{3}}\right. \\
& \left.-k^{2} m_{1} m_{3} \frac{1}{r_{13}}-k^{2} m_{2} m_{3} \frac{\vec{r}_{3} \cdot \vec{r}_{1}}{r_{23}^{3}}-k^{2} \frac{m_{0} m_{2} m_{3}}{m_{0}+m_{2}} \frac{\vec{r}_{0} \cdot \vec{r}_{1}}{r_{23}^{3}}\right] \\
& =\frac{\partial}{\partial x_{1}}\left(k^{2} \frac{m_{0}+m_{1}}{r_{01}}+k^{2} \frac{m_{2}}{r_{12}}+k^{2} \frac{m_{3}}{r_{13}}+k^{2} m_{2} \frac{\vec{r}_{0} \cdot \vec{r}_{1}}{r_{02}^{3}}-k^{2} m_{3} \vec{r}_{1} \cdot \vec{r}_{3}^{\prime \prime} \frac{1}{r_{03}^{3}}\right) .
\end{aligned}
$$

Therefore, upon comparing Equations 12 and 15 we find

$$
U_{\text {GRAV }}=\frac{k^{2} m_{1}}{r_{01}}+\frac{k^{2} m_{2}}{r_{12}}+\frac{k^{2} m_{3}}{r_{13}}+k^{2} m_{2} \frac{\vec{r}_{0} \cdot \vec{r}_{1}}{r_{02}^{3}}-k^{2} m_{3} \frac{\vec{r}_{1} \cdot \vec{r}_{3}^{\prime \prime}}{r_{03}^{3}} .
$$

where

$$
\begin{aligned}
& \vec{r}_{3}^{\prime \prime}=\vec{r}_{3}---\frac{m_{2}}{m_{0}+m_{2}} \vec{r}_{0}, \\
& r_{01}=r_{1}, \\
& r_{02}=r_{0} \cdot \\
& r_{13}=\text { series in Legendre polynomials, }
\end{aligned}
$$

and

$$
\mathrm{r}_{03}=\text { series in Legendre polynomials. }
$$

The term $\mathrm{k}^{2} \mathrm{~m}_{1} / \mathrm{r}_{01}$ can, of course, be neglected. Finally, from Equations 9, 11, and 16,

$$
\begin{aligned}
\widetilde{F}=\frac{\mu_{0}^{2}}{2 \mathrm{~L}^{2}}+\frac{\mu_{2}}{\mathrm{r}_{12}}+\frac{\mu_{3}}{\mathrm{r}_{13}}+\mu_{2} \frac{\overrightarrow{\mathrm{r}}_{0} \cdot \overrightarrow{\mathrm{r}}_{1}}{\mathrm{r}_{0}^{3}}-\frac{\mu_{3} \overrightarrow{\mathrm{r}}_{1} \cdot \overrightarrow{\mathrm{r}}_{3}^{\prime \prime}}{\mathrm{r}_{03}^{3}} \\
+\frac{\mu_{0}}{\mathrm{r}_{1}}\left[-\sum_{\mathrm{n}=2}^{5}\left(\frac{\mathrm{R}_{\mathbb{C}}}{\mathrm{r}_{1}}\right)^{\mathrm{n}} \mathrm{J}_{\mathrm{n}} \mathrm{P}_{\mathrm{n}}(\sin \beta)+\left(\frac{\mathrm{R}_{\mathbb{C}}}{\mathrm{r}_{1}}\right)^{2} \mathrm{~J}_{22} \mathrm{P}_{22}(\sin \beta) \cos 2\left(\lambda^{\prime}-\lambda_{22}\right)\right] .
\end{aligned}
$$


Now

$$
\frac{\vec{r}_{0} \cdot \vec{r}_{1}}{r_{0}^{3}}=\frac{-r_{0} r_{1} \cos S_{10}^{\prime}}{r_{0}^{3}}=-\frac{r_{1}}{r_{0}^{2}} \cos S_{10}^{\prime}
$$

and

$$
\frac{1}{r_{12}}=\frac{1}{r_{0}}+\frac{r_{1}}{r_{0}^{2}} \cos S_{10}^{\prime}+\frac{1}{r_{0}} \sum_{p=2}^{\infty} P_{p}\left(\cos S_{10}^{\prime}\right)\left(\frac{r_{1}}{r_{0}}\right)^{p} .
$$

Then,

$$
\frac{\mu_{2}}{\mathrm{r}_{12}}+\mu_{2} \frac{\overrightarrow{\mathrm{r}}_{0} \cdot \overrightarrow{\mathrm{r}}_{1}}{\mathrm{r}_{0}{ }^{3}}=\frac{\mu_{2}}{\mathrm{r}_{0}}+\frac{\mu_{2}}{\mathrm{r}_{0}} \sum_{\mathrm{p}=2}^{\infty}\left(\frac{\mathrm{r}_{1}}{\mathrm{r}_{0}}\right)^{\mathrm{P}} \mathrm{P}_{\mathrm{p}}\left(\cos \mathrm{S}_{10}^{\prime}\right)
$$

and since $\mu_{2} / r_{0}$ is independent of the position of the orbiter, Equation 17 becomes

$$
\begin{aligned}
\hat{\mathrm{F}}=\frac{\mu_{0}^{2}}{2 \mathrm{~L}^{2}}+\frac{\mu_{2}}{\mathrm{r}_{0}} \sum_{\mathrm{p}=2}^{x}\left(\frac{\mathrm{r}_{1}}{\mathrm{r}_{0}}\right)^{\mathrm{P}} & \mathrm{P}_{\mathrm{p}}\left(\cos \mathrm{S}_{10}^{\prime}\right)+\frac{\mu_{3}}{\mathrm{r}_{13}}-\mu_{3} \frac{\dot{\mathrm{r}}_{1} \cdot \overrightarrow{\mathrm{r}}_{3}^{\prime \prime}}{\mathrm{r}_{03}^{3}} \\
+ & \frac{\mu_{0}}{\mathrm{r}_{1}}\left[-\sum_{\mathrm{n}=2}^{5}\left(\frac{\mathrm{R}_{\overparen{C}}}{\mathrm{r}_{1}}\right)^{\mathrm{n}} \mathrm{J}_{\mathrm{n}} \mathrm{P}_{\mathrm{n}}\left(\sin ()^{\prime}\right)+\left(\frac{\mathrm{R}_{\mathbb{C}}}{\mathrm{r}_{1}}\right)^{2} \mathrm{~J}_{22} \mathrm{P}_{22}(\sin \hat{\beta}) \cos 2\left(\lambda^{\prime}-\lambda_{22}\right)\right] .
\end{aligned}
$$

A further simplification can be made if one considers the following expansion:

$$
\begin{aligned}
r_{13}^{2} & -\left[x_{1}-\left(x_{3}-\frac{m_{2}}{m_{0}{ }^{+} m_{2}} x_{0}\right)\right]^{2}+\cdots \\
& =r_{1}^{2}+r_{03}^{2}-2 r_{1} r_{03} \cos S_{13}^{\prime \prime}=r_{03}^{2}\left[1+\left(\frac{r_{1}}{r_{03}}\right)^{2}-2 \frac{r_{1}}{r_{03}} \cos S_{13}^{\prime \prime}\right] .
\end{aligned}
$$

Then,

$$
\begin{aligned}
\frac{1}{r_{13}} & =\frac{1}{r_{03}} \sum_{p=0}^{\alpha}\left(\frac{r_{1}}{r_{03}}\right)^{p} P_{p}\left(\cos S_{13}^{\prime \prime}\right) \\
& =\frac{1}{r_{03}}+\frac{r_{1} \cos S_{13}^{\prime \prime}}{r_{03}^{2}}+\frac{1}{r_{03}} \sum_{p=2}^{\infty}\left(\frac{r_{1}}{r_{03}}\right)^{p} P_{p}\left(\cos S_{13}^{\prime \prime}\right) .
\end{aligned}
$$


Also,

$$
\frac{\vec{r}_{1} \cdot \vec{r}_{3}^{\prime \prime}}{r_{03}^{3}}=\underset{r_{03}^{3} r_{03} \cos S_{13}^{\prime \prime}}{r_{03}^{3}}=\frac{r_{1} \frac{\cos S_{13}^{\prime \prime}}{r_{03}^{2}}}{.} .
$$

Since $r_{03}$ is independent of $x_{1}, y_{1}, z_{1}$, Equation 18 becomes

$\widetilde{F}=\frac{\mu_{0}^{2}}{2 L^{2}}+\frac{\mu_{2}}{r_{0}} \sum_{p=2}^{\infty}\left(\frac{r_{1}}{r_{0}}\right)^{p} P_{p}\left(\cos S_{10}^{\prime}\right)+\frac{\mu_{3}}{r_{03}} \sum_{p=2}^{\infty}\left(\frac{r_{1}}{r_{03}}\right)^{p} P_{p}\left(\cos S_{13}^{\prime \prime}\right)$

$$
+\frac{\mu_{0}}{r_{1}}\left[-\sum_{n=2}^{5}\left(\frac{R_{\overparen{C}}}{r_{1}}\right)^{n} J_{n} P_{n}(\sin \beta)+\left(\frac{R_{\overparen{C}}}{r_{1}}\right)^{2} J_{22} P_{22}(\sin \beta) \cos 2\left(\lambda^{\prime}-\lambda_{22}\right)\right] .
$$

From now on, the subscript 1 will be omitted.

Including terms of second order, the Hamiltonian becomes

$$
\begin{aligned}
\widetilde{\mathrm{F}} \frac{\mu_{0}{ }^{2}}{2 \mathrm{~L}^{2}}+\frac{\mu_{2}}{\mathrm{r}_{0}}\left(\frac{\mathrm{r}}{\mathrm{r}_{0}}\right)^{2}\left[\mathrm{P}_{2}\left(\cos \mathrm{S}_{10}^{\prime}\right)+\frac{\mathrm{r}}{\mathrm{r}_{0}} \mathrm{P}_{3}\left(\cos \mathrm{S}_{10}^{\prime}\right)\right]+\frac{\mu_{3}}{\mathrm{r}_{3}}\left(\frac{\mathrm{r}}{\mathrm{r}_{3}}\right)^{2} \mathrm{P}_{2}\left(\cos \mathrm{S}_{13}^{\prime \prime}\right) \\
+\frac{\mu_{0}}{\mathrm{r}}\left[-\sum_{\mathrm{n}=2}^{5}\left(\frac{\mathrm{R}_{\mathbb{C}}}{\mathrm{r}}\right)^{\mathrm{n}} \mathrm{J}_{\mathrm{n}} \mathrm{P}_{\mathrm{n}}(\sin s)+\left(\frac{\mathrm{R}_{\mathbb{C}}}{\mathrm{r}}\right)^{2} \mathrm{~J}_{22} \mathrm{P}_{22}(\sin \rho) \cos 2\left(\lambda^{\prime}-\lambda_{22}\right)\right] .
\end{aligned}
$$

\section{THE ANGLES $\mathbf{S}_{10}^{\prime}$ AND $\mathbf{S}_{13}^{\prime \prime}$}

In view of the form assigned to the oblateness terms, the plane of reference is the equator of the moon. Therefore, the next step is to express the angles $S_{10}^{\prime}$ and $S_{13}^{\prime \prime}$ in terms of the orbital elements of the orbiter, the moon, the earth, and the sun, with respect to that plane. The geometry is shown in Figure 2.

The explicit form of $S_{10}^{\prime}$ and $S_{13}^{\prime \prime}$ requires the solution of two spherical quadrangles. This is now done.

If $\mathrm{x}, \mathrm{y}, \mathrm{z}$ and $\mathrm{x}_{\oplus}, \mathrm{y}_{\oplus}, \mathrm{z}_{\oplus}$ are the rectangular coordinates of the orbiter and the earth, respectively, and $r$ and $r_{\oplus}$ their selenocentric distances, then

$$
\cos S_{10}^{\prime}=\frac{\vec{r} \cdot \vec{r}_{\oplus}}{r r_{\oplus}} .
$$




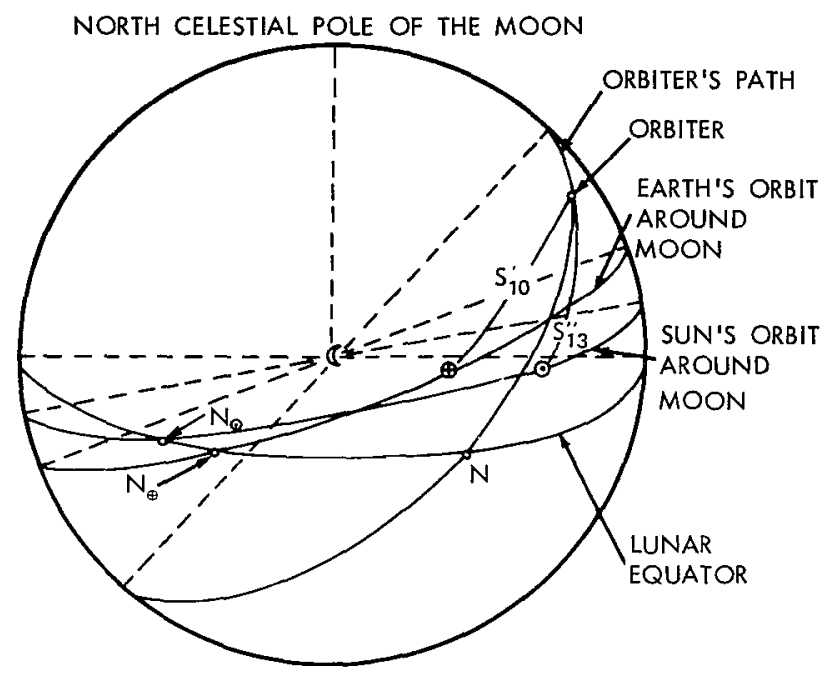

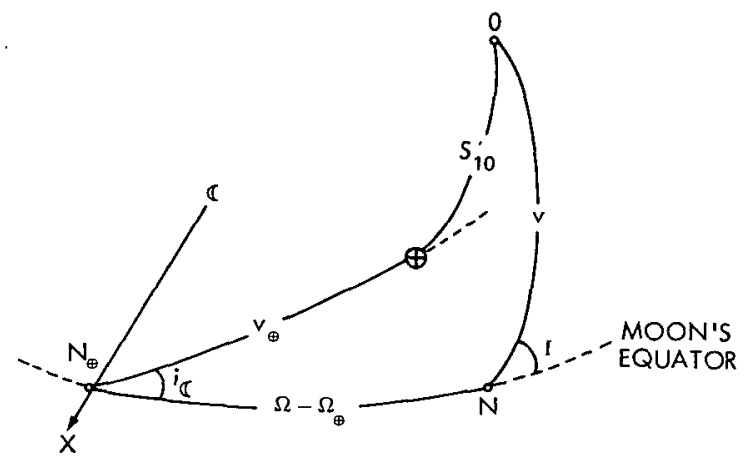

Figure 3-Angular relationships.

Figure 2-Selenocentric celestial sphere.

Consider

$$
\begin{gathered}
\Delta \Omega=\Omega-\Omega_{\oplus}, \\
\mathrm{v}=\mathrm{f}+\mathrm{a}, \text { and } \mathrm{v}_{\oplus}=\mathrm{f}_{\oplus}+a_{\oplus} .
\end{gathered}
$$

Then, using vector notation, in the equatorial (moon) system indicated in Figure 3,

$$
\begin{aligned}
& \vec{r}_{\oplus}=r_{\oplus}\left(\begin{array}{c}
\cos v_{\oplus} \\
\sin v_{\oplus} \cos i_{\mathbb{C}} \\
\sin v_{\oplus} \sin i_{\mathbb{C}}
\end{array}\right) \text {. } \\
& \vec{r}=r\left(\begin{array}{c}
(\cos a \cos \Delta \Omega-\sin \omega \sin \Delta \Omega \cos I) \cos f-(\sin \omega \cos \Delta \Omega+\cos \omega \sin \Delta \Omega \cos I) \sin f \\
(\cos \omega \sin \Delta \Omega+\sin \omega \cos \Delta \Omega \cos I) \cos f-(\sin \omega \sin \Delta \Omega-\cos \omega \cos \Delta \Omega \cos I) \sin f \\
\sin \omega \sin I \cos f+\cos \omega \sin I \sin f
\end{array}\right) .
\end{aligned}
$$

This enables us to compute $\cos \mathrm{S}_{10}^{\prime}$.

In exactly the same way, $\cos \mathrm{S}_{13}{ }_{3}$ is obtained by substituting $\odot$ for $\oplus$ in the formulas in this section.

\section{THE MAIN PROBLEM}

The following approximations are made:

a. The earth's orbit around the moon (or vice versa) lies on the lunar equatorial plane. The error introduced by this approximation is proportional to the sine of half the inclination of the lunar orbit to its equator $\left(\sim 6^{\circ} 41^{\prime}\right)$, or about 0.06 . 
b. The orbit of the moon around the earth is circular and the motion uniform. The error is proportional to the moon's eccentricity, or about 0.055 .

c. The sun's perturbations are negligible. The relative error for a moderately high satellite is about 0.05 .

d. The mean longitude of the earth, $\lambda_{\oplus}$, is equal to $\lambda_{22}$.

With these approximations, the precision of the disturbing function is not higher than $10^{-4}$. It will be called the "disturbing function of the main problem," and it is given by

$$
\begin{aligned}
\widetilde{\mathrm{F}}=\frac{\mu_{0}^{2}}{2 \mathrm{~L}^{2}}+\frac{\mathrm{n}_{0}^{2}}{\epsilon} \mathrm{r}^{2} \mathrm{P}_{2}\left(\cos \widetilde{\mathrm{S}}_{10}^{\prime}\right) & +\frac{\mu_{0}}{\mathrm{r}}\left\{\left(\frac{\mathrm{R}_{\mathbb{C}}}{\mathrm{r}}\right)^{2}\left[-\mathrm{J}_{2} \mathrm{P}_{2}(\sin \beta)+\mathrm{J}_{22} \mathrm{P}_{22}(\sin \beta) \cos 2\left(\lambda^{\prime}-\lambda_{\oplus}\right)\right]\right. \\
& \left.-\left(\frac{\mathrm{R}_{\mathbb{C}}}{\mathrm{r}}\right)^{3} \mathrm{~J}_{3} \mathrm{P}_{3}(\sin \beta)-\left(\frac{\mathrm{R}_{\mathbb{C}}}{\mathrm{r}}\right)^{4} \mathrm{~J}_{4} \mathrm{P}_{4}(\sin \beta)-\left(\frac{\mathrm{R}_{\overparen{C}}}{\mathrm{r}}\right)^{5} \mathrm{~J}_{5} \mathrm{P}_{5}(\sin \beta)\right\},
\end{aligned}
$$

where

$$
-1+\frac{\mu_{0}}{\mu_{2}}
$$

and where the angle $\widetilde{\mathrm{S}}_{10}^{\prime}$ is as shown in Figure 4. Since $i_{\overparen{C}}=0$ and $v_{\oplus}+\Omega_{\oplus}$ can be replaced by $\lambda_{\oplus}$, it then follows that

$$
\cos \widetilde{S}_{10}^{\prime}=\cos v \cos \left(\Omega_{1}-\lambda_{4}\right)-\sin v \sin \left(S_{i}-\lambda_{f}\right) \cos I-s .
$$

The equations of motion are given by the canonical set

$$
\begin{array}{ll}
\dot{L}=\frac{\partial \widetilde{\mathrm{F}}}{\partial l}, & \dot{i}=-\frac{\partial \widetilde{\widetilde{F}}}{\partial \mathrm{L}}, \\
\dot{\mathrm{G}}=\frac{\partial \widetilde{\widetilde{F}}}{\partial c}, & \dot{\omega}=-\frac{\partial \widetilde{\mathrm{F}}}{\partial \mathrm{G}}, \\
\dot{\mathbf{H}}=\frac{\partial \widetilde{\mathrm{F}}}{\partial \Omega}, & \dot{\Omega}=-\frac{\partial \widetilde{\mathrm{F}}}{\partial \mathrm{H}} .
\end{array}
$$

Since the variables $\Omega$ and $\lambda_{\oplus}$ appear only in the combination $\Omega-\lambda_{\oplus}$, where $\lambda_{\oplus}=n_{\mathbb{C}}^{*} t+$ const., the degree of freedom is reduced by one by choosing

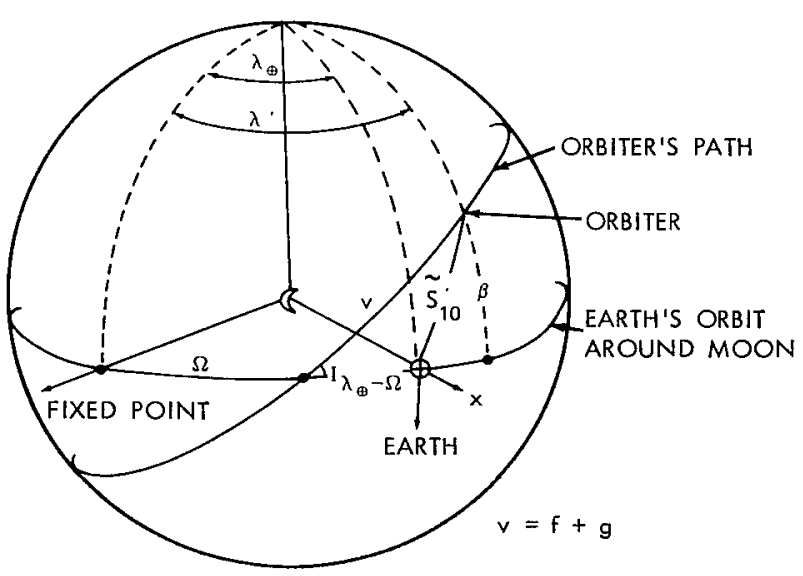

Figure 4-Simplified selenocentric celestial sphere. 
as a new variable

$$
\mathrm{h}=\Omega-\lambda_{\oplus} .
$$

The Hamiltonian must be modified accordingly, for

$$
\dot{\mathrm{h}}=\dot{\Omega}-\mathrm{n}_{\mathbb{C}}^{*}=-\frac{\partial}{\partial \mathrm{H}}\left(\widetilde{\widetilde{F}}+\mathrm{n}_{\mathbb{C}}^{*} \mathrm{H}\right)
$$

(i.e., upon substitution of $\tilde{\widetilde{F}}+n_{\mathbb{C}}^{*} H$ in place of $\tilde{\widetilde{F}}$ ).

The Hamiltonian is still time-dependent through $\lambda_{\oplus}$. Since the longest meridian is always pointing toward the earth, it is possible to choose the rotating system whose $x$-axis passes through this meridian. The final form of the Hamiltonian for the main problem is therefore

$$
\begin{aligned}
\mathrm{F}=\frac{\mu_{0}^{2}}{2 \mathrm{~L}^{2}}+\mathrm{n}_{\mathbb{C}}^{*} \mathrm{H}+\frac{\mathrm{n}_{\mathbb{C}}^{2} \mathrm{r}^{2}}{\epsilon} \mathrm{P}_{2}(\mathrm{~s}) & +\frac{\mu_{0}}{\mathrm{r}}\left\{\left(\frac{\mathrm{R}_{\mathbb{C}}}{\mathrm{r}}\right)^{2}\left[-\mathrm{J}_{2} \mathrm{P}_{2}(\sin \beta)+\mathrm{J}_{22} \mathrm{P}_{22}(\sin \beta) \cos 2 \lambda\right]\right. \\
& -\left(\frac{\mathrm{R}_{\mathbb{C}}}{\mathrm{r}}\right)^{3} \mathrm{~J}_{3} \mathrm{P}_{3}\left(\sin (s)-\left(\frac{\mathrm{R}_{\overparen{C}}}{\mathrm{r}}\right)^{4} \mathrm{~J}_{4} \mathrm{P}_{4}(\sin \beta)-\left(\frac{\mathrm{R}_{\mathbb{C}}}{\mathrm{r}}\right)^{5} \mathrm{~J}_{5} \mathrm{P}_{5}(\sin (\beta)\},\right.
\end{aligned}
$$

where

$$
\lambda=\lambda^{\prime}-\lambda_{\oplus} .
$$

The equations of motion are (using $g$ in place of $\omega$ ):

$$
\begin{array}{ll}
\dot{\mathrm{L}}=\frac{\partial \mathrm{F}}{\partial i}, \quad \dot{\mathrm{G}}=\frac{\partial \mathrm{F}}{\partial \mathrm{g}}, \quad \dot{\mathrm{H}}=\frac{\partial \mathrm{F}}{\partial \mathrm{h}} . \\
\dot{i}=-\frac{\partial \mathrm{F}}{\partial \mathrm{L}}, \quad \dot{\mathrm{g}}=-\frac{\partial \mathrm{F}}{\partial \mathrm{G}}, \quad \dot{\mathrm{h}}=-\frac{\partial \mathrm{F}}{\partial \mathrm{H}} .
\end{array}
$$

In the discussion that follows, the solution of this system will be given using von Zeipel's method.

\section{DEVELOPMENT OF THE DISTURBING FUNCTION}

The notation used will be as shown in Figure 4.

From Equation 24,

$$
\cos \widetilde{S}_{10}^{\prime}=s=\cos \left(v+\Omega-\lambda_{\oplus}\right)-2 \sin ^{2} \frac{I}{2} \sin v \sin \left(\lambda_{\oplus}-\Omega\right)
$$


thus,

$$
\begin{aligned}
P_{2}(s)= & \frac{3}{2} s^{2}-\frac{1}{2} \\
& =\frac{3}{2}\left\{\cos ^{2} f \cos (2 g+2 h)+\sin ^{2}(g+h)\right. \\
& -\sin f \cos f \sin (2 g+2 h)+4 \sin ^{4} \frac{I}{2} \sin ^{2} h\left[\cos ^{2} g-\cos ^{2} f \cos 2 g\right. \\
& +\sin f \cos f \sin 2 g]+4 \sin ^{2} \frac{I}{2} \sinh [\sin f \cos f \cos (2 g+h)
\end{aligned}
$$

$$
\left.\left.-\cos g \sin (g+h)+\cos ^{2} f \sin (2 g+h)\right]\right\}-\frac{1}{2} .
$$

\section{Further,}

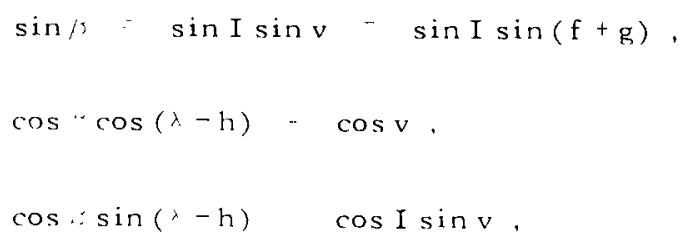

and therefore

$$
\cos \cdot \cos \lambda=\cos v \cos h-\cos I \sin v \sinh \text {. }
$$

from which

$P_{2}\left(\sin :^{\circ}\right) \quad \frac{3}{2} \sin ^{2},-\frac{1}{2} \quad \frac{3}{2} \sin ^{2} I \sin ^{2}(f+g)-\frac{1}{2}$,

$P_{22}(\sin i s) \cos 2 \lambda-6 \cos ^{2} \beta \cos ^{2} \lambda-3 \cos ^{2} \beta$

$$
=6\left(\zeta^{2} \cos ^{2} f+\chi^{2} \sin ^{2} f+2 \zeta \chi \sin f \cos f\right)-3\left(1-\sin ^{2} I \sin ^{2} v\right)
$$

$P_{3}(\sin \beta)=\frac{5}{2} \sin ^{3} \beta-\frac{3}{2} \sin \beta=\frac{5}{2} \sin ^{3} I \sin ^{3}(f+g)-\frac{3}{2} \sin I \sin (f+g)$,

$P_{4}(\sin \beta)=\frac{35}{8} \sin ^{4} \beta-\frac{15}{4} \sin ^{2} \beta+\frac{3}{8}=\frac{35}{8} \sin ^{4} I \sin ^{4}(f+g)-\frac{15}{4} \sin ^{2} I \sin ^{2}(f+g)+\frac{3}{8}$,

$P_{5}(\sin \beta)=\frac{63}{8} \sin ^{5} \beta-\frac{35}{4} \sin ^{3} \beta+\frac{15}{8} \sin \beta=\frac{63}{8} \sin ^{5} I \sin ^{5}(f+g)-\frac{35}{4} \sin ^{3} I \sin ^{3}(f+g)+\frac{15}{8} \sin I \sin (f+g)$ 
where

$$
\zeta=\cos g \cos h-\cos I \sin g \sinh
$$

and

$$
\chi=-\sin g \cos h-\cos I \cos g \sinh .
$$

Therefore, writing $\mu_{\overparen{C}}$ for $\mu_{0}$ in Equation 26, we have

$$
\begin{aligned}
& F=\frac{\mu_{\mathbb{C}}^{2}}{2 L^{2}}+n_{\mathbb{C}}^{*} H+\frac{3}{2} \frac{n_{\mathbb{C}}^{2} r^{2}}{\epsilon}\left\{\cos ^{2} f \cos (2 g+2 h)+\sin ^{2}(g+h)-\sin f \cos f \sin (2 g+2 h)\right. \\
& +4 \sin ^{4} \frac{I}{2} \sin ^{2} h\left[\cos ^{2} g-\cos ^{2} f \cos 2 g+\sin f \cos f \sin 2 g\right]+4 \sin ^{2} \frac{I}{2} \sinh [\sin f \cos f \cos (2 g+h) \\
& \left.\left.-\cos g \sin (g+h)+\cos ^{2} f \sin (2 g+h)\right]\right\}-\frac{1}{2 \epsilon} n_{\mathbb{C}}^{2} r^{2}+\frac{\mu_{\mathbb{C}} R_{\mathbb{C}}^{2}}{r^{3}}\left\{-\frac{3}{2} J_{2} \sin ^{2} I \sin ^{2}(f+g)\right. \\
& \left.+\frac{1}{2} J_{2}+J_{22}\left[6\left(\zeta^{2} \cos ^{2} f+\chi^{2} \sin ^{2} f+2 \zeta \chi \sin f \cos f\right)-3\left(1-\sin ^{2} I \sin ^{2} v\right)\right]\right\} \\
& -\frac{\mu_{\mathbb{C}} R_{\mathbb{C}}^{3} J_{3}}{r^{4}}\left[\frac{5}{2} \sin ^{3} I \sin ^{3}(f+g)-\frac{3}{2} \sin I \sin (f+g)\right]-\frac{\mu_{C} R_{C}^{4} J_{4}}{r^{5}}\left[\frac{35}{8} \sin ^{4} I \sin ^{4}(f+g)\right. \\
& \left.-\frac{15}{4} \sin ^{2} I \sin ^{2}(f+g)+\frac{3}{8}\right]-\frac{\mu_{\overparen{C}} R_{\overparen{C}}^{5} J}{r^{6}}\left[\frac{63}{8} \sin ^{5} I \sin ^{5}(f+g)-\frac{35}{4} \sin ^{3} I \sin ^{3}(f+g)\right. \\
& \left.+\frac{15}{8} \sin I \sin (f+g)\right] \\
& =F_{0}+F_{1}+F_{2} \text {, }
\end{aligned}
$$

\section{where}

$$
\begin{aligned}
& \mathrm{F}_{0}=\frac{\mu_{\mathbb{C}}^{2}}{2 \mathrm{~L}^{2}} \quad \text { (0th order), } \\
& \mathrm{F}_{1}=\mathrm{n}_{\mathbb{C}}^{*} \mathrm{H} \text { (1st order), }
\end{aligned}
$$


and

$$
F_{2}=F-\left(F_{1}+F_{0}\right) \quad(\text { 2nd order })
$$

\section{ELIMINATION OF SHORT-PERIOD PERTURBATIONS}

The terms in $\mathrm{F}$ which depend on $l$ will result in short-period perturbations. According to von Zeipel's method, the elimination of these terms corresponds to the solution of the system

$$
\begin{aligned}
F_{0}^{\prime}\left(L^{\prime}\right) & =F_{0}\left(L^{\prime}\right), \\
F_{1}^{\prime}\left(H^{\prime}\right) & =F_{1}\left(H^{\prime}\right)+\frac{\partial S_{1}}{\partial l} \frac{\partial F_{0}}{\partial L^{\prime}}, \\
F_{2}^{\prime} & =F_{2}+\frac{\partial S_{1}}{\partial h} \frac{\partial F_{1}}{\partial H^{\prime}}+\frac{\partial S_{2}}{\partial l} \frac{\partial F_{0}}{\partial L^{\prime}}+\frac{1}{2}\left(\frac{\partial S_{1}}{\partial l}\right)^{2} \frac{\partial^{2} F_{0}}{\partial L^{\prime 2}} \cdots,
\end{aligned}
$$

where the generating function of the transformation

$$
(\mathrm{L}, \mathrm{G}, \mathrm{H}, l, \mathrm{~g}, \mathrm{~h}) \rightarrow\left(\mathrm{L}^{\prime}, \mathrm{G}^{\prime}, \mathrm{H}^{\prime}, l^{\prime}, \mathrm{g}^{\prime}, \mathrm{h}^{\prime}\right)
$$

is

$$
\mathrm{S} \quad \mathrm{L}^{\prime} l+\mathrm{G}^{\prime} \mathrm{g}+\mathrm{H}^{\prime} \mathrm{h}+\mathrm{S}_{1}+\mathrm{S}_{2}+\cdots,
$$

and the new Hamiltonian

$$
\mathrm{F}^{\prime} \quad \mathrm{F}_{0}^{\prime} \cdot \mathrm{F}_{1}^{\prime}+\mathrm{F}_{2}^{\prime}+\cdots
$$

should be independent of $l^{\prime}$.

In order to accomplish this last requirement, a particular solution is given by

$$
\begin{array}{ll}
\mathrm{S}_{1} & 0, \\
\mathrm{~F}_{2}^{\prime} & \mathrm{F}_{2 \mathrm{~s}} .
\end{array}
$$

and

$$
\frac{\partial \mathrm{S}_{2}}{\partial l} \frac{\partial \mathbf{F}_{0}}{\partial \mathrm{L}^{\prime}}=-\mathbf{F}_{2 \mathrm{p}}
$$

where $F_{2 s}$ and $F_{2 p}$ are, respectively, the parts of $F_{2}$ independent of $l$ and dependent on $l$. 
If $\mathrm{s}_{2}$ is determined from this system, then the relations between old and new variables are

$$
\begin{aligned}
& l^{\prime}=l+\frac{\partial \mathrm{S}_{2}}{\partial \mathrm{L}^{\prime}}, \quad \mathrm{g}^{\prime}=\mathrm{g}+\frac{\partial \mathrm{S}_{2}}{\partial \mathrm{G}^{\prime}}, \quad \mathrm{h}^{\prime}=\mathrm{h}+\frac{\partial \mathrm{S}_{2}}{\partial \mathrm{H}^{\prime}}, \\
& \mathrm{L}=\mathrm{L}^{\prime}+\frac{\partial \mathrm{S}_{2}}{\partial l}, \quad \mathrm{G}=\mathrm{G}^{\prime}+\frac{\partial \mathrm{S}_{2}}{\partial \mathrm{g}}, \quad \mathrm{H}=\mathrm{H}^{\prime}+\frac{\partial \mathrm{S}_{2}}{\partial \mathrm{h}} .
\end{aligned}
$$

Next, the "secular" part of $\mathrm{F}_{2}$ is determined. By definition,

$$
\mathrm{F}_{2 \mathrm{~s}}=\frac{1}{2 \pi} \int_{0}^{2 \pi} \mathrm{F}_{2} \mathrm{~d} l
$$

Using the relations ( $E=$ eccentric anomaly)

$$
\begin{aligned}
& d l=(1-e \cos E) d E, \\
& r \cos f-a(\cos E-e), \\
& r \operatorname{sinf} \quad a \sqrt{1-e^{2}} \sin E, \\
& r=a(1-e \cos E), \\
& d l \quad=\frac{a^{2}\left(1-e^{2}\right)^{1 / 2} d f,}{}
\end{aligned}
$$

and

$$
\frac{1}{r} \quad \frac{1+e \cos f}{a\left(1-e^{2}\right)} .
$$

it follows that

$$
\begin{aligned}
\mathrm{F}^{\prime}= & \frac{\mu_{\mathbb{C}}^{2}}{2 \mathrm{~L}^{\prime 2}}+\mathrm{n}_{\mathbb{C}}^{*} \mathrm{H}^{\prime}+\frac{\mathrm{n}_{\mathscr{C}}^{2} \mathrm{~L}^{\prime 4}}{16_{\mathrm{C}_{\mathbb{C}}^{2}}^{2} \in}\left\{\left(5-3 \frac{\mathrm{G}^{\prime 2}}{\mathrm{~L}^{\prime 2}}\right)\left[\left(-1+3 \frac{\mathrm{H}^{\prime 2}}{\mathrm{G}^{\prime 2}}\right)+3\left(1-\frac{\mathrm{H}^{\prime 2}}{\mathrm{G}^{\prime 2}}\right) \cos 2 \mathrm{~h}^{\prime}\right]\right. \\
& \left.+15\left(1-\frac{\mathrm{G}^{\prime 2}}{\mathrm{~L}^{\prime 2}}\right)\left[\frac{1}{2}\left(1+\frac{\mathrm{H}^{\prime}}{\mathrm{G}^{\prime}}\right)^{2} \cos 2\left(\mathrm{~g}^{\prime}+\mathrm{h}^{\prime}\right)+\left(1-\frac{\mathrm{H}^{\prime 2}}{\mathrm{G}^{\prime 2}}\right) \cos 2 \mathrm{~g}^{\prime}+\frac{1}{2}\left(1-\frac{\mathrm{H}^{\prime}}{\mathrm{G}^{\prime}}\right)^{2} \cos 2\left(\mathrm{~g}^{\prime}-\mathrm{h}^{\prime}\right)\right]\right\} \\
& +\frac{1}{4} \frac{\mu_{\mathbb{C}}^{4}}{\mathrm{~L}^{\prime 6}}\left(\frac{\mathrm{L}^{\prime}}{\mathrm{G}^{\prime}}\right)^{3}\left[-\mathrm{R}_{\mathbb{C}}^{2} \mathrm{~J}_{2}\left(1-3 \frac{\mathrm{H}^{\prime 2}}{\mathrm{G}^{\prime 2}}\right)+6 \mathrm{R}_{\mathbb{C}}^{2} \mathrm{~J}_{22}\left(1-\frac{\mathrm{H}^{\prime 2}}{\mathrm{G}^{\prime 2}}\right) \cos 2 \mathrm{~h}^{\prime}\right]
\end{aligned}
$$




$$
\begin{aligned}
& -\frac{3}{8} \frac{\mu_{C}^{5} R_{\mathbb{C}}^{3} \mathrm{~J}_{3}}{\mathrm{~L}^{\prime 8}}\left(\frac{\mathrm{L}^{\prime}}{\mathrm{G}^{\prime}}\right)^{5}\left(1-\frac{\mathrm{G}^{\prime 2}}{\mathrm{~L}^{\prime 2}}\right)^{1 / 2}\left(1-5 \frac{\mathrm{H}^{\prime 2}}{\mathrm{G}^{\prime 2}}\right)\left(1-\frac{\mathrm{H}^{\prime 2}}{\mathrm{G}^{\prime 2}}\right)^{1 / 2} \sin \mathrm{g}^{\prime} \\
& -\frac{3}{128} \frac{\mu_{C}^{6} \mathrm{R}_{\mathbb{C}}^{4} \mathrm{~J}_{4}}{\mathrm{~L}^{\prime 10}}\left(\frac{\mathrm{L}^{\prime}}{\mathrm{G}^{\prime}}\right)^{7}\left[\left(5-3 \frac{\mathrm{G}^{\prime 2}}{\mathrm{~L}^{\prime 2}}\right)\left(3-30 \frac{\mathrm{H}^{\prime 2}}{\mathrm{G}^{\prime 2}}+35 \frac{\mathrm{H}^{\prime 4}}{\mathrm{G}^{\prime 4}}\right)-10\left(1-\frac{\mathrm{G}^{\prime 2}}{\mathrm{~L}^{\prime 2}}\right)\left(1-\frac{\mathrm{H}^{\prime 2}}{\mathrm{G}^{\prime 2}}\right)\left(1-7 \frac{\mathrm{H}^{\prime 2}}{\mathrm{G}^{\prime 2}}\right) \cos 2 \mathrm{~g}^{\prime}\right] \\
& -\frac{5}{256} \frac{\mu_{\mathbb{C}}^{7} \mathrm{R}_{\mathbb{C}}^{5} \mathrm{~J}_{5}}{\mathrm{~L}^{\prime 12}}\left(\frac{\mathrm{L}^{\prime}}{\mathrm{G}^{\prime}}\right)^{9}\left(1-\frac{\mathrm{G}^{\prime 2}}{\mathrm{~L}^{\prime 2}}\right)^{1 / 2}\left(1-\frac{\mathrm{H}^{\prime 2}}{\mathrm{G}^{\prime 2}}\right)^{1 / 2}\left[6\left(7-3 \frac{\mathrm{G}^{\prime 2}}{\mathrm{~L}^{\prime 2}}\right)\left(1-14 \frac{\mathrm{H}^{\prime 2}}{\mathrm{G}^{\prime 2}}+21 \frac{\mathrm{H}^{\prime 4}}{\mathrm{G}^{\prime 4}}\right) \sin \mathrm{g}^{\prime}\right. \\
& \left.-7\left(1-\frac{\mathrm{G}^{\prime 2}}{\mathrm{~L}^{\prime 2}}\right)\left(1-\frac{\mathrm{H}^{\prime 2}}{\mathrm{G}^{\prime 2}}\right)\left(1-9 \frac{\mathrm{H}^{\prime 2}}{\mathrm{G}^{\prime 2}}\right) \sin 3 \mathrm{~g}^{\prime}\right] \cdot(39)
\end{aligned}
$$

Also, since

$$
\dot{L}^{\prime}=\frac{\partial F^{\prime}}{\partial l^{\prime}}=0
$$

then

$$
L^{\prime}=\text { const. }
$$

The generating function $S_{2}$ and the short-period perturbations are obtained next. Since

$$
\frac{\partial F_{0}}{\partial L^{\prime}}--\frac{\mu_{C}^{2}}{L^{\prime 3}}=-n^{\prime}
$$

from Equation 35 it follows that

$$
\mathrm{S}_{2}=\frac{1}{\mathrm{n}^{\prime}} \int \mathrm{F}_{2 \mathrm{p}} \mathrm{d} l=\frac{1}{\mathrm{n}^{\prime}} \int\left(\mathrm{F}_{2}-\mathrm{F}_{2 \mathrm{~s}}\right) \mathrm{d} l
$$

The integration is carried out using $\mathrm{E}$ or $\mathrm{f}$ as independent variables, again making use of Equations 38. As a result,

$$
\begin{aligned}
S_{2}= & \frac{1}{8} n_{n^{\prime} a^{\prime 3}\left(1-e^{\prime 2}\right)^{3 / 2}}^{\mu_{\mathbb{C}} R_{\overparen{C}}^{2} J_{2}}\left[-2\left(1-3 \cos ^{2} I^{\prime}\right) B_{2,1}+\left(\sin ^{2} I^{\prime}\right) B_{2,2}\right] \\
& +\frac{1}{8} n_{n^{\prime} a^{\prime 3}\left(1-e^{\prime 2}\right)^{3 / 2}}^{\mu_{C} R_{\mathbb{C}}^{2} J_{22}}\left[6\left(\sin ^{2} I^{\prime}\right) B_{22,1}+\left(1-\cos I^{\prime}\right)^{2} B_{22,2}+\left(1+\cos I^{\prime}\right)^{2} B_{22,3}\right]
\end{aligned}
$$




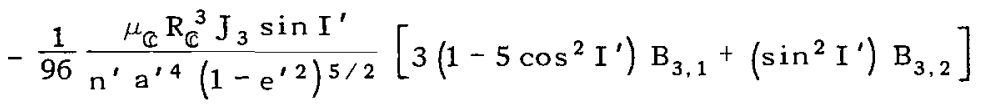

$$
\begin{aligned}
& +\frac{1}{512} \frac{\mu_{C} R_{\mathbb{C}}^{4} J_{4}}{n^{\prime} a^{\prime 5}\left(1-e^{\prime 2}\right)^{7 / 2}}\left[-2\left\langle 3-30 \cos ^{2} I^{\prime}+35 \cos ^{4} I^{\prime}\right) B_{4,1}\right. \\
& \left.+2 \sin ^{2} I^{\prime}\left(1-7 \cos ^{2} I^{\prime}\right) B_{4,2}-\left(\sin ^{4} I^{\prime}\right) B_{4,3}\right] \\
& -\frac{1}{30720} \frac{\mu_{\overparen{C}} R_{\mathbb{C}}^{5} J_{5} \sin I^{\prime}}{n^{\prime} a^{\prime 6}\left(1-e^{\prime 2}\right)^{9 / 2}}\left[30\left(1-14 \cos ^{2} I^{\prime}+21 \cos ^{4} I^{\prime}\right) B_{5,1}+5 \sin ^{2} I^{\prime}\left(1-9 \cos ^{2} I^{\prime}\right) B_{5,2}-3\left(\sin ^{4} I^{\prime}\right) B_{5,3}\right] \\
& +\frac{1}{384} \frac{n_{C^{2} a^{\prime 2}}}{\epsilon n^{\prime}}\left\{1 2 \left[-2\left(1-3 \cos ^{2} I^{\prime}\right)\left(2+3 e^{\prime 2}\right)+6 \sin ^{2} I^{\prime}\left(2+3 e^{\prime 2}\right) \cos 2 h+30 e^{\prime 2} \sin ^{2} I^{\prime} \cos 2 g\right.\right. \\
& \left.+15 e^{\prime 2}\left(1-\cos I^{\prime}\right)^{2} \cos (2 g-2 h)+15 e^{\prime 2}\left(1+\cos I^{\prime}\right)^{2} \cos (2 g+2 h)\right] \cdot\left(E^{\prime}-l\right) \\
& \left.+4\left(1-3 \cos ^{2} I^{\prime}\right) B_{\overparen{C}, 1}+6\left(\sin ^{2} I^{\prime}\right) B_{\overparen{C}, 2}+3\left(1-\cos I^{\prime}\right)^{2} B_{\mathbb{C}, 3}+3\left(1+\cos I^{\prime}\right)^{2} B_{\mathbb{C}, 4}\right\}
\end{aligned}
$$

where

$$
\begin{aligned}
& B_{2,1}=e^{\prime} \sin f^{\prime}+\left(f^{\prime}-l\right), \\
& B_{2,2}=3 e^{\prime} \sin \left(f^{\prime}+2 g\right)+3 \sin \left(2 f^{\prime}+2 g\right)+e^{\prime} \sin \left(3 f^{\prime}+2 g\right), \\
& B_{22,1}=2\left(f^{\prime}-l\right) \cos 2 h+e^{\prime} \sin \left(f^{\prime}-2 h\right) e^{\prime} \sin \left(f^{\prime}+2 h\right), \\
& B_{22,2}=3 e^{\prime} \sin \left(f^{\prime}+2 g-2 h\right)+3 \sin \left(2 f^{\prime}+2 g-2 h\right)+e^{\prime} \sin \left(3 f^{\prime}+2 g-2 h\right), \\
& B_{22,3}=3 e^{\prime} \sin \left(f^{\prime}+2 g+2 h\right)+3 \sin \left(2 f^{\prime}+2 g+2 h\right)+e^{\prime} \sin \left(3 f^{\prime}+2 g+2 h\right), \\
& B_{3,1}=12 e^{\prime}\left(f^{\prime}-l\right) \sin g+3 e^{\prime 2} \cos \left(f^{\prime}-g\right)-6\left(2+e^{\prime 2}\right) \cos \left(f^{\prime}+g\right)-6 e^{\prime} \cos \left(2 f^{\prime}+g\right)-e^{\prime 2} \cos \left(3 f^{\prime}+g\right), \\
& B_{3,2}=15 e^{\prime 2} \cos \left(f^{\prime}+3 g\right)+30 e^{\prime} \cos \left(2 f^{\prime}+3 g\right)+10\left(2+e^{\prime 2}\right) \cos \left(3 f^{\prime}+3 g\right) \\
& B_{4,1}=6\left(2+3 e^{\prime 2}\right)\left(f^{\prime}-l\right)+9 e^{\prime}\left(4+e^{\prime 2}\right) \sin f^{\prime}+9 e^{\prime 2} \sin 2 f^{\prime}+e^{\prime 3} \sin 3 f^{\prime},
\end{aligned}
$$




$$
\begin{aligned}
& B_{4,2}=60 e^{\prime 2}\left(f^{\prime}-l\right) \cos 2 g+10 e^{\prime 3} \sin \left(f^{\prime}-2 g\right)+30 e^{\prime}\left(4+e^{\prime 2}\right) \sin \left(f^{\prime}+2 g\right)+20\left(2+3 e^{\prime 2}\right) \sin \left(2 f^{\prime}+2 g\right) \\
& +10 e^{\prime}\left(4+e^{\prime 2}\right) \sin \left(3 f^{\prime}+2 g\right)+15 e^{\prime 2} \sin \left(4 f^{\prime}+2 g\right)+2 e^{\prime 3} \sin \left(5 f^{\prime}+2 g\right) \\
& B_{4,3}=35 e^{\prime 3} \sin \left(f^{\prime}+4 g\right)+105 e^{\prime 2} \sin \left(2 f^{\prime}+4 g\right)+35 e^{\prime}\left(4+e^{\prime 2}\right) \sin \left(3 f^{\prime}+4 g\right)+35\left(2+3 e^{\prime 2}\right) \sin \left(4 f^{\prime}+4 g\right) \\
& +21 e^{\prime}\left(4+e^{\prime 2}\right) \sin \left(5 f^{\prime}+4 g\right)+35 e^{\prime 2} \sin \left(6 f^{\prime}+4 g\right)+5 e^{\prime 3} \sin \left(7 f^{\prime}+4 g\right) \\
& B_{5,1}=120 e^{\prime}\left(4+3 e^{\prime 2}\right)\left(f^{\prime}-l\right) \sin g-30\left(8+24 e^{\prime 2}+3 e^{\prime 4}\right) \cos \left(f^{\prime}+g\right)+60 e^{\prime 2}\left(6+e^{\prime 2}\right) \cos \left(f^{\prime}-g\right) \\
& -60 e^{\prime}\left(4+3 e^{\prime 2}\right) \cos \left(2 f^{\prime}+g\right)+60 e^{\prime 3} \cos \left(2 f^{\prime}-g\right) \\
& -20 e^{\prime 2}\left(6+e^{\prime 2}\right) \cos \left(3 f^{\prime}+g\right)+5 e^{\prime 4} \cos \left(3 f^{\prime}-g\right)-30 e^{\prime 3} \cos \left(4 f^{\prime}+g\right)-3 e^{\prime 4} \cos \left(5 f^{\prime}+g\right), \\
& B_{5,2}=-840 e^{\prime 3}\left(f^{\prime}-l\right) \sin 3 g+420 e^{\prime 2}\left(6+e^{\prime 2}\right) \cos \left(f^{\prime}+3 g\right)-105 e^{\prime 4} \cos \left(f^{\prime}-3 g\right) \\
& +420 e^{\prime}\left(4+3 e^{\prime 2}\right) \cos \left(2 f^{\prime}+3 g\right)+70\left(8+24 e^{\prime 2}+3 e^{\prime 4}\right) \cos \left(3 f^{\prime}+3 g\right)+210 e^{\prime}\left(4+3 e^{\prime 2}\right) \cos \left(4 f^{\prime}+3 g\right) \\
& +84 e^{\prime 2}\left(6+e^{\prime 2}\right) \cos \left(5 f^{\prime}+3 g\right)+140 e^{\prime 3} \cos \left(6 f^{\prime}+3 g\right)+15 e^{\prime 4} \cos \left(7 f^{\prime}+3 g\right) \\
& B_{5,3}=315 e^{\prime 4} \cos \left(f^{\prime}+5 g\right)+1260 e^{\prime 3} \cos \left(2 f^{\prime}+5 g\right)+420 e^{\prime 2}\left(6+e^{\prime 2}\right) \cos \left(3 f^{\prime}+5 g\right) \\
& +630 e^{\prime}\left(4+3 e^{\prime 2}\right) \cos \left(4 f^{\prime}+5 g\right)+126\left(8+24 e^{\prime 2}+3 e^{\prime 4}\right) \cos \left(5 f^{\prime}+5 g\right)+420 e^{\prime}\left(4+3 e^{\prime 2}\right) \cos \left(6 f^{\prime}+5 g\right) \\
& +180 e^{\prime 2}\left(6+e^{\prime 2}\right) \cos \left(7 f^{\prime}+5 g\right)+315 e^{\prime 3} \cos \left(8 f^{\prime}+5 g\right)+35 e^{\prime 4} \cos \left(9 f^{\prime}+5 g\right)
\end{aligned}
$$$$
B_{\mathbb{C}, 1}=9 e^{\prime}\left(4+e^{\prime 2}\right) \sin E^{\prime}-9 e^{\prime 2} \sin 2 E^{\prime}+e^{\prime 3} \sin 3 E^{\prime},
$$$$
B_{\mathbb{C}, 2}=-9 e^{\prime}\left(4+e^{\prime 2}\right) \sin \left(E^{\prime}-2 h\right)+9 e^{\prime 2} \sin \left(2 E^{\prime}-2 h\right)-e^{\prime 3} \sin \left(3 E^{\prime}-2 h\right)
$$$$
-9 e^{\prime}\left(4+e^{\prime 2}\right) \sin \left(E^{\prime}+2 h\right)+9 e^{\prime 2} \sin \left(2 E^{\prime}+2 h\right)-e^{\prime 3} \sin \left(3 E^{\prime}+2 h\right)
$$$$
-15 e^{\prime}\left[\left(2+e^{\prime 2}\right)-2\left(1-e^{\prime 2}\right)^{1 / 2}\right] \sin \left(E^{\prime}-2 g\right)+3\left[\left(2+e^{\prime 2}\right)-2\left(1-e^{\prime 2}\right)^{1 / 2}\left(1+e^{\prime 2}\right)\right] \sin \left(2 E^{\prime}-2 g\right)
$$$$
-e^{\prime}\left[\left(2-e^{\prime 2}\right)-2\left(1-e^{\prime 2}\right)^{1 / 2}\right] \sin \left(3 E^{\prime}-2 g\right)-15 e^{\circ}\left[\left(2+e^{\prime 2}\right)+2\left(1-e^{\prime 2}\right)^{1 / 2}\right] \sin \left(E^{\prime}+2 g\right)
$$$$
+3\left[\left(2+e^{\prime 2}\right)+2\left(1-e^{\prime 2}\right)^{1 / 2}\left(1+e^{\prime 2}\right)\right] \sin \left(2 E^{\prime}+2 g\right)-e^{\prime}\left[\left(2-e^{\prime 2}\right)+2\left(1-e^{\prime 2}\right)^{1 / 2}\right] \sin \left(3 E^{\prime}+2 g\right)
$$ 


$$
\begin{aligned}
\mathrm{B}_{\mathbb{C}, 3}= & -15 \mathrm{e}^{\prime}\left[\left(2+\mathrm{e}^{\prime 2}\right)-2\left(1-\mathrm{e}^{\prime 2}\right)^{1 / 2}\right] \sin \left(\mathrm{E}^{\prime}-2 \mathrm{~g}+2 \mathrm{~h}\right)+3\left[\left(2+\mathrm{e}^{\prime 2}\right)\right. \\
& \left.-2\left(1-\mathrm{e}^{\prime 2}\right)^{1 / 2}\left(1+\mathrm{e}^{\prime 2}\right)\right] \sin \left(2 \mathrm{E}^{\prime}-2 \mathrm{~g}+2 \mathrm{~h}\right)-\mathrm{e}^{\prime}\left[\left(2-\mathrm{e}^{\prime 2}\right)-2\left(1-\mathrm{e}^{\prime 2}\right)^{1 / 2}\right] \sin \left(3 \mathrm{E}^{\prime}-2 \mathrm{~g}+2 \mathrm{~h}\right) \\
- & 15 \mathrm{e}^{\prime}\left[\left(2+\mathrm{e}^{\prime 2}\right)+2\left(1-\mathrm{e}^{\prime 2}\right)^{1 / 2}\right] \sin \left(\mathrm{E}^{\prime}+2 \mathrm{~g}-2 \mathrm{~h}\right)+3\left[\left(2+\mathrm{e}^{\prime 2}\right)+2\left(1-\mathrm{e}^{\prime 2}\right)^{1 / 2}\left(1+\mathrm{e}^{\prime 2}\right)\right] \sin \left(2 \mathrm{E}^{\prime}+2 \mathrm{~g}-2 \mathrm{~h}\right) \\
& -\mathrm{e}^{\prime}\left[\left(2-\mathrm{e}^{\prime 2}\right)+2\left(1-\mathrm{e}^{\prime 2}\right)^{1 / 2}\right] \sin \left(3 \mathrm{E}^{\prime}+2 \mathrm{~g}-2 \mathrm{~h}\right) .
\end{aligned}
$$

and

$$
\begin{aligned}
\mathrm{B}_{\mathscr{C}, 4}= & -15 \mathrm{e}^{\prime}\left[\left(2+\mathrm{e}^{\prime 2}\right)-2\left(1-\mathrm{e}^{\prime 2}\right)^{1 / 2}\right] \sin \left(\mathrm{E}^{\prime}-2 \mathrm{~g}-2 \mathrm{~h}\right)+3\left[\left(2+\mathrm{e}^{\prime 2}\right)\right. \\
& \left.-2\left(1-\mathrm{e}^{\prime 2}\right)^{1 / 2}\left(1+\mathrm{e}^{\prime 2}\right)\right] \sin \left(2 \mathrm{E}^{\prime}-2 \mathrm{~g}-2 \mathrm{~h}\right)-\mathrm{e}^{\prime}\left[\left(2-\mathrm{e}^{\prime 2}\right)-2\left(1-\mathrm{e}^{\prime 2}\right)^{1 / 2}\right] \sin \left(3 \mathrm{E}^{\prime}-2 \mathrm{~g}-2 \mathrm{~h}\right) \\
& -15 \mathrm{e}^{\prime}\left[\left(2+\mathrm{e}^{\prime 2}\right)+2\left(1-\mathrm{e}^{\prime 2}\right)^{1 / 2}\right] \sin \left(\mathrm{E}^{\prime}+2 \mathrm{~g}+2 \mathrm{~h}\right)+3\left[\left(2+\mathrm{e}^{\prime 2}\right)+2\left(1-\mathrm{e}^{\prime 2}\right)^{1 / 2}\left(1+\mathrm{e}^{\prime 2}\right)\right] \sin \left(2 \mathrm{E}^{\prime}+2 \mathrm{~g}+2 \mathrm{~h}\right) \\
& -\mathrm{e}^{\prime}\left[\left(2-\mathrm{e}^{\prime 2}\right)+2\left(1-\mathrm{e}^{\prime 2}\right)^{1 / 2}\right] \sin \left(3 \mathrm{E}^{\prime}+2 \mathrm{~g}+2 \mathrm{~h}\right) \cdot(42)
\end{aligned}
$$

Thus, for the short-period terms,

$$
\mathrm{S}=\mathrm{L}^{\prime} l+\mathrm{G}^{\prime} \mathrm{g}+\mathrm{H}^{\prime} \mathrm{h}+\mathrm{S}_{2}
$$

to second order.

The short-period perturbations are given by

$$
\begin{aligned}
& l^{\prime}=\frac{\partial \mathrm{S}}{\partial \mathrm{L}^{\prime}}=l+\frac{\partial \mathrm{S}_{2}}{\partial \mathrm{L}^{\prime}}, \\
& \mathrm{g}^{\prime}=\frac{\partial \mathrm{S}}{\partial \mathrm{G}^{\prime}}=\mathrm{g}+\frac{\partial \mathrm{S}_{2}}{\partial \mathrm{G}^{\prime}}, \\
& \mathrm{h}^{\prime}=\frac{\partial \mathrm{S}}{\partial \mathrm{H}^{\prime}}=\mathrm{h}+\frac{\partial \mathrm{S}_{2}}{\partial \mathrm{H}^{\prime}}, \\
& \mathrm{L}=\frac{\partial \mathrm{S}}{\partial l}=\mathrm{L}^{\prime}+\frac{\partial \mathrm{S}_{2}}{\partial l}, \\
& \mathrm{G}=\frac{\partial \mathrm{S}}{\partial \mathrm{g}}=\mathrm{G}^{\prime}+\frac{\partial \mathrm{S}_{2}}{\partial \mathrm{g}},
\end{aligned}
$$


and

$$
H=\frac{\partial S}{\partial h}=H^{\prime}+\frac{\partial S_{2}}{\partial h} .
$$

It is easier to compute the partial derivatives with respect to the Keplerian elements $a^{\prime}, e^{\prime}, I^{\prime}$ and then compute them with respect to $L^{\prime}, \mathrm{G}^{\prime}, \mathrm{H}^{\prime}$, as follows:

$$
\begin{aligned}
& \frac{\partial \mathrm{S}_{2}}{\partial \mathrm{L}^{\prime}}=\frac{\partial \mathrm{S}_{2}}{\partial \mathrm{a}^{\prime}} \frac{\partial \mathrm{a}^{\prime}}{\partial \mathrm{L}^{\prime}}+\frac{\partial \mathrm{S}_{2}}{\partial \mathrm{e}^{\prime}} \frac{\partial \mathrm{e}^{\prime}}{\partial \mathrm{L}^{\prime}}=2 \frac{\mathrm{L}^{\prime}}{\mu_{\mathbb{C}}} \frac{\partial \mathrm{S}_{2}}{\partial \mathrm{a}^{\prime}}+\frac{\mathrm{G}^{\prime 2}}{\mathrm{e}^{\prime} \mathrm{L}^{\prime 3}} \frac{\partial \mathrm{S}_{2}}{\partial \mathrm{e}^{\prime}}, \\
& \frac{\partial \mathrm{S}_{2}}{\partial \mathrm{G}^{\prime}}=\frac{\partial \mathrm{S}_{2}}{\partial \mathrm{e}^{\prime}} \frac{\partial \mathrm{e}^{\prime}}{\partial \mathrm{G}^{\prime}}+\frac{\partial \mathrm{S}_{2}}{\partial \mathrm{I}^{\prime}} \frac{\partial \mathrm{I}^{\prime}}{\partial \mathrm{G}^{\prime}}=-\frac{\mathrm{G}^{\prime}}{\mathrm{e}^{\prime} \mathrm{L}^{\prime 2}} \frac{\partial \mathrm{S}_{2}}{\partial \mathrm{e}^{\prime}}+\frac{\mathrm{H}^{\prime}}{\mathrm{G}^{\prime 2} \sin \mathrm{I}^{\prime}} \frac{\partial \mathrm{S}_{2}}{\partial \mathrm{I}^{\prime}},
\end{aligned}
$$

and

$$
\frac{\partial \mathrm{S}_{2}}{\partial \mathrm{H}^{\prime}} \quad \frac{\partial \mathrm{S}_{2}}{\partial \mathrm{I}^{\prime}} \frac{\partial \mathrm{I}^{\prime}}{\partial \mathrm{H}^{\prime}}=-\frac{1}{\mathrm{G}^{\prime} \sin \mathrm{I}^{\prime}} \frac{\partial \mathrm{S}_{2}}{\partial \mathrm{I}^{\prime}} .
$$

Furthermore, it is important to note that

$$
\begin{aligned}
& \frac{\partial f^{\prime}}{\partial e^{\prime}}=\left(\frac{a^{\prime}}{r^{\prime}}+\frac{L^{\prime 2}}{G^{\prime 2}}\right) \sin f^{\prime}, \\
& \frac{\partial E^{\prime}}{\partial e^{\prime}}=\frac{a^{\prime}}{r^{\prime}} \sin E^{\prime},
\end{aligned}
$$

and

$$
\frac{\partial n^{\prime}}{\partial a^{\prime}}=-\frac{3}{2} \frac{n^{\prime}}{a^{\prime}} .
$$

The partial derivative with respect to $l$ does not need to be computed again, since

$$
\frac{\partial S_{2}}{\partial l}=\frac{1}{n^{\prime}} F_{2 p}=\frac{1}{n^{\prime}}\left(F-F^{\prime}\right) .
$$


Thus,

$$
\begin{aligned}
& \frac{\partial S_{2}}{\partial a^{\prime}}=-\frac{3}{16} \frac{n^{\prime} R_{C}^{2} J_{2}}{a^{\prime}\left(1-e^{\prime 2}\right)^{3 / 2}}\left[-2\left(1-3 \cos ^{2} I^{\prime}\right) B_{2,1}+\left(\sin ^{2} I^{\prime}\right) B_{2,2}\right] \\
& -\frac{3}{16} \frac{\mathrm{n}^{\prime} \mathrm{R}_{\mathbb{C}}^{2} \mathrm{~J}_{22}}{\mathrm{a}^{\prime}\left(1-\mathrm{e}^{\prime 2}\right)^{3 / 2}}\left[6\left(\sin ^{2} I^{\prime}\right) \mathrm{B}_{22,1}+\left(1-\cos \mathrm{I}^{\prime}\right)^{2} \mathrm{~B}_{22,2}+\left(1+\cos \mathrm{I}^{\prime}\right)^{2} \mathrm{~B}_{22,3}\right] \\
& +\frac{5}{192} \frac{n^{\prime} R_{\mathbb{C}}^{3} J_{3} \sin I^{\prime}}{a^{\prime 2}\left(1-e^{\prime 2}\right)^{5 / 2}}\left[3\left(1-5 \cos ^{2} I^{\prime}\right) B_{3,1}+\left(\sin ^{2} I^{\prime}\right) B_{3,2}\right] \\
& -\frac{7}{1024} \frac{\mathrm{n}^{\prime} \mathrm{R}_{\mathbb{C}}^{4} J_{4}}{\mathrm{a}^{\prime 3}\left(1-\mathrm{e}^{\prime 2}\right)^{7 / 2}}\left[-2\left(3-30 \cos ^{2} \mathrm{I}^{\prime}+35 \cos ^{4} \mathrm{I}^{\prime}\right) \mathrm{B}_{4,1}\right. \\
& \left.+2 \sin ^{2} I^{\prime}\left(1-7 \cos ^{2} I^{\prime}\right) B_{4,2}-\left(\sin ^{4} I^{\prime}\right) B_{4,3}\right] \\
& +\frac{9}{61440} \frac{n^{\prime} R_{C}^{5} J_{5} \sin I^{\prime}}{a^{\prime 4}\left(1-e^{\prime 2}\right)^{9 / 2}}\left[30\left(1-14 \cos ^{2} I^{\prime}+21 \cos ^{4} I^{\prime}\right) B_{5,1}\right. \\
& \left.+5 \sin ^{2} I^{\prime}\left(1-9 \cos ^{2} I^{\prime}\right) B_{5,2}-3\left(\sin ^{4} I^{\prime}\right) B_{5,3}\right] \\
& +\frac{7}{768} \frac{\mathrm{n}_{\mathbb{C}}^{2} \mathrm{a}^{\prime}}{\epsilon \mathrm{n}^{\prime}}\left\{1 2 \left[-2\left(1-3 \cos ^{2} \mathrm{I}^{\prime}\right)\left(2+3 \mathrm{e}^{\prime 2}\right)+6 \sin ^{2} \mathrm{I}^{\prime}\left(2+3 \mathrm{e}^{\prime 2}\right) \cos 2 \mathrm{~h}\right.\right. \\
& \left.+30 e^{\prime 2} \sin ^{2} I^{\prime} \cos 2 g+15 e^{\prime 2}\left(1-\cos I^{\prime}\right)^{2} \cos (2 g-2 h)+15 e^{\prime 2}\left(1+\cos I^{\prime}\right)^{2} \cos (2 g+2 h)\right]\left(E^{\prime}-l\right) \\
& \left.+4\left(1-3 \cos ^{2} I^{\prime}\right) B_{\mathbb{C}, 1}+6\left(\sin ^{2} I^{\prime}\right) B_{\mathbb{C}, 2}+3\left(1-\cos I^{\prime}\right)^{2} B_{\mathbb{C}, 3}+3\left(1+\cos I^{\prime}\right)^{2} B_{\mathbb{C}, 4}\right\} \\
& \frac{\partial S_{2}}{\partial I^{\prime}}=\frac{1}{4} \frac{n^{\prime} R_{C}^{2} J_{2}}{\left(1-e^{\prime 2}\right)^{3 / 2}} \sin I^{\prime} \cos I^{\prime}\left(-6 B_{2,1}+B_{2,2}\right) \\
& +\frac{1}{4} \frac{n^{\prime} R_{\overparen{C}}^{2} J_{22}}{\left(1-e^{\prime 2}\right)^{3 / 2}} \sin I^{\prime}\left[6\left(\cos I^{\prime}\right) B_{22,1}+\left(1-\cos I^{\prime}\right) B_{22,2}-\left(I+\cos I^{\prime}\right) B_{22,3}\right] \\
& -\frac{1}{32} \frac{n^{\prime} R_{\mathbb{C}}^{3} J_{3} \cos I^{\prime}}{a^{\prime}\left(1-e^{\prime 2}\right)^{5 / 2}}\left[\left(11-15 \cos ^{2} I^{\prime}\right) B_{3,1}+\left(\sin ^{2} I^{\prime}\right) B_{3,2}\right]
\end{aligned}
$$




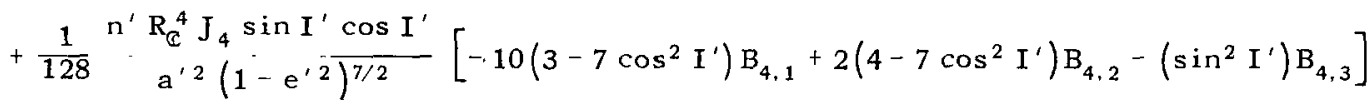

$$
\begin{aligned}
& -\frac{1}{2048} \frac{\Pi^{\prime} R_{\overparen{C}}^{5} J_{5} \cos I^{\prime}}{a^{\prime 3}\left(1-e^{\prime 2}\right)^{9 / 2}}\left[2\left(29-126 \cos ^{2} I^{\prime}+105 \cos ^{4} I^{\prime}\right) B_{5,1}\right. \\
& \left.+\sin ^{2} I^{\prime}\left(7-15 \cos ^{2} I^{\prime}\right) B_{5,2}-\left(\sin ^{4} I^{\prime}\right) B_{5,3}\right] \\
& +\frac{1}{64} \frac{n^{\prime} a^{\prime 2}}{\epsilon}\left(\frac{n c}{n^{\prime}}\right)^{2} \sin I^{\prime}\left\{1 2 \left[-2 \cos I^{\prime}\left(2+3 e^{\prime 2}\right)+2 \cos I^{\prime}\left(2+3 e^{\prime 2}\right) \cos 2 h\right.\right. \\
& \left.+10 e^{\prime 2} \cos I^{\prime} \cos 2 g+5 e^{\prime 2}\left(1-\cos I^{\prime}\right) \cos (2 g-2 h)-5 e^{\prime 2}\left(I+\cos I^{\prime}\right) \cos (2 g+2 h)\right]\left(E^{\prime}-l\right) \\
& \left.+4\left(\cos I^{\prime}\right) B_{\mathbb{C} .1}+2\left(\cos I^{\prime}\right) B_{\mathbb{C} .2}+\left(1-\cos I^{\prime}\right) B_{\mathbb{C} .3}-\left(1+\cos I^{\prime}\right) B_{\mathbb{C} .4}\right\} \\
& \frac{\partial S_{2}}{\partial e^{\prime}}=\frac{1}{32} \frac{n^{\prime} R_{\mathbb{C}}^{2} J_{2}}{\left(1-e^{\prime 2}\right)^{5 / 2}}\left\{-2\left(1-3 \cos ^{2} I^{\prime}\right)\left[12 e^{\prime}\left(f^{\prime}-l\right)+3\left(4+3 e^{\prime 2}\right) \sin f^{\prime}+6 e^{\prime} \sin 2 f^{\prime}+e^{\prime 2} \sin 3 f^{\prime}\right]\right. \\
& \sin ^{2} I^{\prime}\left[-18 e^{\prime} \sin 2 g-3\left(4-7 e^{\prime 2}\right) \sin \left(f^{\prime}+2 g\right)+3 e^{\prime 2} \sin \left(f^{\prime}-2 g\right)+36 e^{\prime} \sin \left(2 f^{\prime}+2 g\right)\right. \\
& \left.\left.+\left(28+11 e^{\prime 2}\right) \sin \left(3 f^{\prime}+2 g\right)+18 e^{\prime} \sin \left(4 f^{\prime}+2 g\right)+3 e^{\prime 2} \sin \left(5 f^{\prime}+2 g\right)\right]\right\} \\
& +\frac{1}{32} \underset{\left(1-e^{\prime 2}\right)^{5^{\prime}}}{n^{\prime} R_{C^{2}} J_{22}}\left\{6 \operatorname { s i n } ^ { 2 } I ^ { \prime } \left[24 e^{\prime}\left(f^{\prime}-7\right) \cos 2 h+3\left(4+3 e^{\prime 2}\right) \sin \left(f^{\prime}+2 h\right)+3\left(4+3 e^{\prime 2}\right) \sin \left(f^{\prime}-2 h\right)\right.\right. \\
& \left.+6 e^{\prime} \sin \left(2 f^{\prime}+2 h\right)+6 e^{\prime} \sin \left(2 f^{\prime}-2 h\right)+e^{\prime 2} \sin \left(3 f^{\prime}+2 h\right)+e^{\prime 2} \sin \left(3 f^{\prime}-2 h\right)\right] \\
& +\left(1-\cos I^{\prime}\right)^{2}\left[-18 e^{\prime} \sin (2 g-2 h)-3\left(4-7 e^{\prime 2}\right) \sin \left(f^{\prime}+2 g-2 h\right)+3 e^{\prime 2} \sin \left(f^{\prime}-2 g+2 h\right)\right. \\
& +36 e^{\prime} \sin \left(2 f^{\prime}+2 g-2 h\right)+\left(28+11 e^{\prime 2}\right) \sin \left(3 f^{\prime}+2 g-2 h\right)+18 e^{\prime} \sin \left(4 f^{\prime}+2 g-2 h\right) \\
& \left.+3 e^{\prime 2} \sin \left(5 f^{\prime}+2 g-2 h\right)\right]+\left(1+\cos I^{\prime}\right)^{2}\left[-18 e^{\prime} \sin (2 g+2 h)-3\left(4-7 e^{\prime 2}\right) \sin \left(f^{\prime}+2 g+2 h\right)\right. \\
& +3 e^{\prime 2} \sin \left(f^{\prime}-2 g-2 h\right)+36 e^{\prime} \sin \left(2 f^{\prime}+2 g+2 h\right)+\left(28+11 e^{\prime 2}\right) \sin \left(3 f^{\prime}+2 g+2 h\right) \\
& \left.\left.+18 e^{\prime} \sin \left(4 f^{\prime}+2 g+2 h\right)+3 e^{\prime 2} \sin \left(5 f^{\prime}+2 g+2 h\right)\right]\right\}
\end{aligned}
$$


$-\frac{1}{128} \frac{n^{\prime} R_{\mathbb{C}}^{3} J_{3} \sin I^{\prime}}{a^{\prime}\left(1-e^{\prime 2}\right)^{7 / 2}}\left\{\left(1-5 \cos ^{2} I^{\prime}\right)\left[48\left(1+4 e^{\prime 2}\right)\left(f^{\prime}-l\right) \sin g+24\left(2+e^{\prime 2}\right) \cos 2 g\right.\right.$

$+42 e^{\prime}\left(2+e^{\prime 2}\right) \cos \left(f^{\prime}-g\right)-72 e^{\prime}\left(4+e^{\prime 2}\right) \cos \left(f^{\prime}+g\right)+24 e^{\prime 2} \cos \left(2 f^{\prime}-g\right)-24\left(3+5 e^{\prime 2}\right) \cos \left(2 f^{\prime}+g\right)$

$\left.+3 e^{\prime 3} \cos \left(3 f^{\prime}-g\right)-2 e^{\prime}\left(34+9 e^{\prime 2}\right) \cos \left(3 f^{\prime}+g\right)-24 e^{\prime 2} \cos \left(4 f^{\prime}+g\right)-3 e^{\prime 3} \cos \left(5 f^{\prime}+g\right)\right]$

$+\sin ^{2} I^{\prime}\left[-40 e^{\prime 2} \cos 3 g-5 e^{\prime 3} \cos \left(f^{\prime}-3 g\right)-10 e^{\prime}\left(6-5 e^{\prime 2}\right) \cos \left(f^{\prime}+3 g\right)-40\left(1-3 e^{\prime 2}\right) \cos \left(2 f^{\prime}+3 g\right)\right.$

$+40 e^{\prime}\left(4+e^{\prime 2}\right) \cos \left(3 f^{\prime}+3 g\right)+20\left(5+6 e^{\prime 2}\right) \cos \left(4 f^{\prime}+3 g\right)+2 e^{\prime}\left(54+11 e^{\prime 2}\right) \cos \left(5 f^{\prime}+3 g\right)$

$$
\left.\left.+40 e^{\prime 2} \cos \left(6 f^{\prime}+3 g\right)+5 e^{\prime 3} \cos \left(7 f^{\prime}+3 g\right)\right]\right\}
$$

$+\frac{1}{2048} \frac{n^{\prime} R_{\overparen{C}}^{4} J_{4}}{a^{\prime 2}\left(1-e^{\prime 2}\right)^{9 / 2}}\left\{-2\left(3-30 \cos ^{2} I^{\prime}+35 \cos ^{4} I^{\prime}\right)\left[120 e^{\prime}\left(4+3 e^{\prime 2}\right)\left(f^{\prime}-7\right)+30\left(8+36 e^{\prime 2}+5 e^{\prime 4}\right) \sin f^{\prime}\right.\right.$

$\left.+240 e^{\prime}\left(1+e^{\prime 2}\right) \sin 2 f^{\prime}+5 e^{\prime 2}\left(24+5 e^{\prime 2}\right) \sin 3 f^{\prime}+30 e^{\prime 3} \sin 4 f^{\prime}+3 e^{\prime 4} \sin 5 f^{\prime}\right]$

$+4 \sin ^{2} I^{\prime}\left(1-7 \cos ^{2} I^{\prime}\right)\left[120 e^{\prime}\left(2+5 e^{\prime 2}\right)\left(f^{\prime}-l\right) \cos 2 g-20 e^{\prime}\left(14+5 e^{\prime 2}\right) \sin 2 g+5 e^{\prime 2}\left(48+19 e^{\prime 2}\right) \sin \left(f^{\prime}-2 g\right)\right.$

$+10\left(8+144 e^{\prime 2}+23 e^{\prime 4}\right) \sin \left(f^{\prime}+2 g\right)+200 e^{\prime}\left(4+3 e^{\prime 2}\right) \sin \left(2 f^{\prime}+2 g\right)+50 e^{\prime 3} \sin \left(2 f^{\prime}-2 g\right)$

$+30\left(8+24 e^{\prime 2}+3 e^{\prime 4}\right) \sin \left(3 f^{\prime}+2 g\right)+5 e^{\prime 4} \sin \left(3 f^{\prime}-2 g\right)+10 e^{\prime}\left(34+25 e^{\prime 2}\right) \sin \left(4 f^{\prime}+2 g\right)$

$\left.+e^{\prime 2}\left(192+31 e^{\prime 2}\right) \sin \left(5 f^{\prime}+2 g\right)+50 e^{\prime 3} \sin \left(6 f^{\prime}+2 g\right)+5 e^{\prime 4} \sin \left(7 f^{\prime}+2 g\right)\right]$

$-\sin ^{4} I^{\prime}\left[-350 e^{\prime 3} \sin 4 g+35 e^{\prime 4} \sin \left(f^{\prime}-4 g\right)-35 e^{\prime 2}\left(24-13 e^{\prime 2}\right) \sin \left(f^{\prime}+4 g\right)-280 e^{\prime}\left(4-5 e^{\prime 2}\right) \sin \left(2 f^{\prime}+4 g\right)\right.$

$-70\left(8-36 e^{\prime 2}-7 e^{14}\right) \sin \left(3 f^{\prime}+4 g\right)+700 e^{\prime}\left(4+3 e^{\prime 2}\right) \sin \left(4 f^{\prime}+4 g\right)+14\left(104+252 e^{\prime 2}+29 e^{\prime 4}\right) \sin \left(5 f^{\prime}+4 g\right)$

$+280 e^{\prime}\left(8+5 e^{\prime 2}\right) \sin \left(6 f^{\prime}+4 g\right)+5 e^{\prime 2}\left(264+37 e^{\prime 2}\right) \sin \left(7 f^{\prime}+4 g\right)+350 e^{\prime 3} \sin \left(8 f^{\prime}+4 g\right)$

$$
\left.\left.+35 e^{\prime 4} \sin \left(9 f^{\prime}+4 g\right)\right]\right\}
$$

$-\frac{1}{8192} \frac{n^{\prime} R_{\mathbb{C}}^{5} J_{5}}{a^{\prime 3}\left(1-e^{\prime 2}\right)^{11 / 2} I^{\prime}}\left\{2\left(1-14 \cos ^{2} I^{\prime}+21 \cos ^{4} I^{\prime}\right)\left[480\left(4+41 e^{\prime 2}+18 e^{\prime 4}\right)\left(f^{\prime}-l\right) \sin g\right.\right.$ 


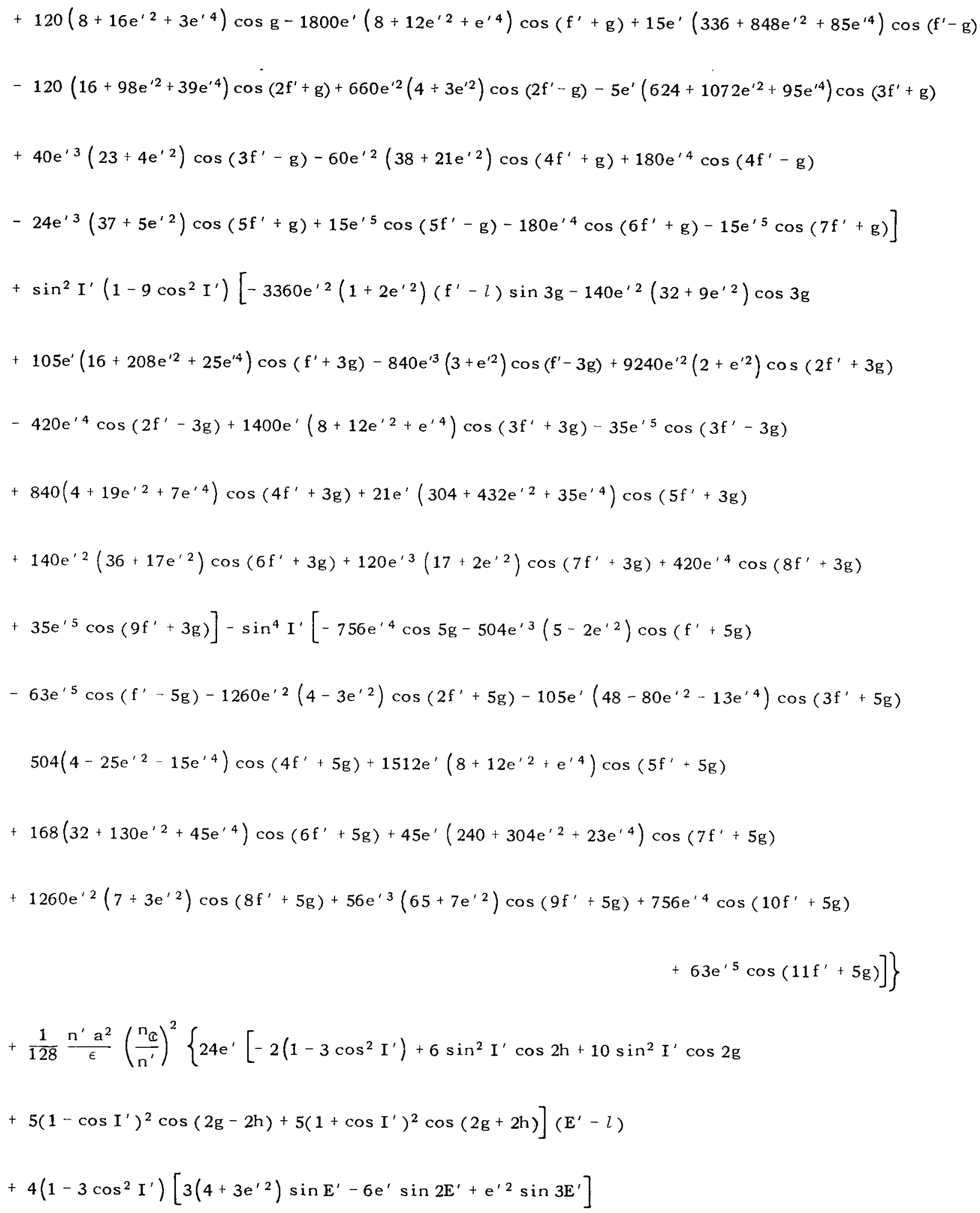




$$
\begin{aligned}
& +2 \sin ^{2} I^{\prime}\left[-9\left(4+3 e^{\prime 2}\right) \sin \left(E^{\prime}-2 h\right)+18 e^{\prime} \sin \left(2 E^{\prime}-2 h\right)-3 e^{\prime 2} \sin \left(3 E^{\prime}-2 h\right)\right. \\
& -9\left(4+3 e^{\prime 2}\right) \sin \left(E^{\prime}+2 h\right)+18 e^{\prime} \sin \left(2 E^{\prime}+2 h\right)-3 e^{\prime 2} \sin \left(3 E^{\prime}+2 h\right) \\
& -15\left\{\left(2+3 e^{\prime 2}\right)-2\left(1-e^{\prime 2}\right)^{1 / 2}+2 e^{\prime 2}\left(1-e^{\prime 2}\right)^{-1 / 2}\right\} \sin \left(E^{\prime}-2 g\right) \\
& +6 e^{\prime}\left\{1-2\left(1-e^{\prime 2}\right)^{1 / 2}+\left(1-e^{\prime 2}\right)^{-1 / 2}\left(1+e^{\prime 2}\right)\right\} \sin \left(2 E^{\prime}-2 g\right) \\
& -\left\{\left(2-3 e^{\prime 2}\right)-2\left(1-e^{\prime 2}\right)^{1 / 2}+2 e^{\prime 2}\left(1-e^{\prime 2}\right)^{-1 / 2}\right\} \sin \left(3 E^{\prime}-2 g\right) \\
& -15\left\{\left(2+3 e^{\prime 2}\right)+2\left(1-e^{\prime 2}\right)^{1 / 2}-2 e^{\prime 2}\left(1-e^{\prime 2}\right)^{-1 / 2}\right\} \sin \left(E^{\prime}+2 g\right) \\
& +6 e^{\prime}\left\{1+2\left(1-e^{\prime 2}\right)^{1 / 2}-\left(1-e^{\prime 2}\right)^{-1 / 2}\left(1+e^{\prime 2}\right)\right\} \sin \left(2 E^{\prime}+2 g\right) \\
& \left.-\left\{\left(2-3 e^{\prime 2}\right)+2\left(1-e^{\prime 2}\right)^{1 / 2}-2 e^{\prime 2}\left(1-e^{\prime 2}\right)^{-1 / 2}\right\} \sin \left(3 E^{\prime}+2 g\right)\right] \\
& \left(1-\cos I^{\prime}\right)^{2}\left[-15\left\{\left(2+3 e^{\prime 2}\right)-2\left(1-e^{\prime 2}\right)^{1 / 2}+2 e^{\prime 2}\left(1-e^{\prime 2}\right)^{-1 / 2}\right\} \sin \left(E^{\prime}-2 g+2 h\right)\right. \\
& +6 e^{\prime}\left\{1-2\left(1-e^{\prime 2}\right)^{1 / 2}+\left(1-e^{\prime 2}\right)^{-1 / 2}\left(1+e^{\prime 2}\right)\right\} \sin \left(2 E^{\prime}-2 g+2 h\right) \\
& -\left\{\left(2-3 e^{\prime 2}\right)-2\left(1-e^{\prime 2}\right)^{1 / 2}+2 e^{\prime 2}\left(1-e^{\prime 2}\right)^{-1 / 2}\right\} \sin \left(3 E^{\prime}-2 g+2 h\right) \\
& -15\left\{\left(2+3 e^{\prime 2}\right)+2\left(1-e^{\prime 2}\right)^{\prime^{\prime} 2}-2 e^{\prime 2}\left(1-e^{\prime 2}\right)^{-1 / 2}\right\} \sin \left(E^{\prime}+2 g-2 h\right) \\
& +6 e^{\prime}\left\{1+2\left(1-e^{\prime 2}\right)^{1 / 2}-\left(1-e^{\prime 2}\right)^{-1 / 2}\left(1+e^{\prime 2}\right)\right\} \sin \left(2 E^{\prime}+2 g-2 h\right) \\
& \left.-\left\{\left(2-3 e^{\prime 2}\right)+2\left(1-e^{\prime 2}\right)^{1 / 2}-2 e^{\prime 2}\left(1-e^{\prime 2}\right)^{-1 / 2}\right\} \sin \left(3 E^{\prime}+2 g-2 h\right)\right] \\
& +\left(1+\cos I^{\prime}\right)^{2}\left[-15\left\{\left(2+3 e^{\prime 2}\right)-2\left(1-e^{\prime 2}\right)^{1 / 2}+2 e^{\prime 2}\left(1-e^{\prime 2}\right)^{-1 / 2}\right\} \sin \left(E^{\prime}-2 g-2 h\right)\right. \\
& +6 e^{\prime}\left\{1-2\left(1-e^{\prime 2}\right)^{l^{\prime} 2}+\left(1-e^{\prime 2}\right)^{-1 / 2}\left(1+e^{\prime 2}\right)\right\} \sin \left(2 E^{\prime}-2 g-2 h\right) \\
& -\left\{\left(2-3 e^{\prime 2}\right)-2\left(1-e^{\prime 2}\right)^{1 / 2}+2 e^{\prime 2}\left(1-e^{\prime 2}\right)^{-1 / 2}\right\} \sin \left(3 E^{\prime}-2 g-2 h\right) \\
& -15\left\{\left(2+3 e^{\prime 2}\right)+2\left(1-e^{\prime 2}\right)^{1 / 2}-2 e^{\prime 2}\left(1-e^{\prime 2}\right)^{-1 / 2}\right\} \sin \left(E^{\prime}+2 g+2 h\right)
\end{aligned}
$$




$$
\begin{aligned}
& +6 e^{\prime}\left\{1+2\left(1-e^{\prime 2}\right)^{1 / 2}-\left(1-e^{\prime 2}\right)^{-1 / 2}\left(1+e^{\prime 2}\right)\right\} \sin \left(2 E^{\prime}+2 g+2 h\right) \\
& \left.\left.-\left\{\left(2-3 e^{\prime 2}\right)+2\left(1-e^{\prime 2}\right)^{1 / 2}-2 e^{\prime 2}\left(1-e^{\prime 2}\right)^{-1 / 2}\right\} \sin \left(3 E^{\prime}+2 g+2 h\right)\right]\right\} \\
& +\frac{1}{384} \frac{n^{\prime} a^{2}}{\epsilon}\left(\frac{n_{\mathbb{C}}}{n^{\prime}}\right)^{2}\left\{1 2 \left[-2\left(1-3 \cos ^{2} I^{\prime}\right)\left(2+3 e^{\prime 2}\right)+6 \sin ^{2} I^{\prime}\left(2+3 e^{\prime 2}\right) \cos 2 h\right.\right. \\
& \left.+30 \mathrm{e}^{\prime 2} \sin ^{2} \mathrm{I}^{\prime} \cos 2 \mathrm{~g}+15 \mathrm{e}^{\prime 2}\left(1-\cos \mathrm{I}^{\prime}\right)^{2} \cos (2 \mathrm{~g}-2 \mathrm{~h})+15 \mathrm{e}^{\prime 2}\left(1+\cos \mathrm{I}^{\prime}\right)^{2} \cos (2 \mathrm{~g}+2 \mathrm{~h})\right] \\
& +12\left(1-3 \cos ^{2} I^{\prime}\right)\left[3 e^{\prime}\left(4+e^{\prime 2}\right) \cos E^{\prime}-6 e^{\prime 2} \cos 2 E^{\prime}+e^{\prime 3} \cos 3 E^{\prime}\right] \\
& +18 \sin ^{2} I^{\prime}\left[-3 e^{\prime}\left(4+e^{\prime 2}\right) \cos \left(E^{\prime}-2 h\right)+6 e^{\prime 2} \cos \left(2 E^{\prime}-2 h\right)-e^{\prime 3} \cos \left(3 E^{\prime}-2 h\right)\right. \\
& -3 e^{\prime}\left(4+e^{\prime 2}\right) \cos \left(E^{\prime}+2 h\right)+6 e^{\prime 2} \cos \left(2 E^{\prime}+2 h\right)-e^{\prime 3} \cos \left(3 E^{\prime}+2 h\right) \\
& -5 e^{\prime}\left\{\left(2+c^{\prime 2}\right)-2\left(1-e^{\prime 2}\right)^{1 / 2}\right\} \cos \left(E^{\prime}-2 g\right) \\
& +2\left\{\left(2+e^{\prime 2}\right)-2\left(1-e^{\prime 2}\right)^{1 / 2}\left(1+e^{\prime 2}\right)\right\} \cos \left(2 E^{\prime}-2 g\right) \\
& -e^{\prime}\left\{\left(2-e^{\prime 2}\right)-2\left(1-e^{\prime 2}\right)^{1 / 2}\right\} \cos \left(3 E^{\prime}-2 g\right) \\
& -5 e^{\prime}\left\{\left(2+e^{\prime 2}\right)+2\left(1-e^{\prime 2}\right)^{1 / 2}\right\} \cos \left(E^{\prime}+2 g\right) \\
& +2\left\{\left(2+e^{\prime 2}\right)+2\left(1-e^{\prime 2}\right)^{1 / 2}\left(1+e^{\prime 2}\right)\right\} \cos \left(2 E^{\prime}+2 g\right) \\
& \left.-e^{\prime}\left\{\left(2-e^{\prime 2}\right)+2\left(1-e^{\prime 2}\right)^{1 / 2}\right\} \cos \left(3 E^{\prime}+2 g\right)\right] \\
& +9\left(1-\cos I^{\prime}\right)^{2}\left[-5 e^{\prime}\left\{\left(2+e^{\prime 2}\right)-2\left(1-e^{\prime 2}\right)^{1 / 2}\right\} \cos \left(E^{\prime}-2 g+2 h\right)\right. \\
& +2\left\{\left(2+e^{\prime 2}\right)-2\left(1-e^{\prime 2}\right)^{1 / 2}\left(1+e^{\prime 2}\right)\right\} \cos \left(2 E^{\prime}-2 g+2 h\right) \\
& -e^{\prime}\left\{\left(2-e^{\prime 2}\right)-2\left(1-e^{\prime 2}\right)^{1 / 2}\right\} \cos \left(3 E^{\prime}-2 g+2 h\right) \\
& -5 e^{\prime}\left\{\left(2+e^{\prime 2}\right)+2\left(1-e^{\prime 2}\right)^{1 / 2}\right\} \cos \left(E^{\prime}+2 g-2 h\right) \\
& +2\left\{\left(2+\mathrm{e}^{\prime 2}\right)+2\left(1-\mathrm{e}^{\prime 2}\right)^{1 / 2}\left(1+\mathrm{e}^{\prime 2}\right)\right\} \cos \left(2 \mathrm{E}^{\prime}+2 \mathrm{~g}-2 \mathrm{~h}\right)
\end{aligned}
$$




$$
\begin{aligned}
& \left.-e^{\prime}\left\{\left(2-e^{\prime 2}\right)+2\left(1-e^{\prime 2}\right)^{1 / 2}\right\} \cos \left(3 E^{\prime}+2 g-2 h\right)\right] \\
& +9\left(1+\cos I^{\prime}\right)^{2}\left[-5 e^{\prime}\left\{\left(2+e^{\prime 2}\right)-2\left(1-e^{\prime^{2}}\right)^{1 / 2}\right\} \cos \left(E^{\prime}-2 g-2 h\right)\right. \\
& +2\left\{\left(2+e^{\prime 2}\right)-2\left(1-e^{\prime 2}\right)^{1 / 2}\left(1+e^{\prime 2}\right)\right\} \cos \left(2 E^{\prime}-2 g-2 h\right) \\
& -e^{\prime}\left\{\left(2-e^{\prime 2}\right)-2\left(1-e^{\prime 2}\right)^{1 / 2}\right\} \cos \left(3 E^{\prime}-2 g-2 h\right) \\
& -5 e^{\prime}\left\{\left(2+e^{\prime 2}\right)+2\left(1-e^{\prime 2}\right)^{1 / 2}\right\} \cos \left(E^{\prime}+2 g+2 h\right) \\
& +2\left\{\left(2+e^{\prime 2}\right)+2\left(1-e^{\prime 2}\right)^{1 / 2}\left(1+e^{\prime 2}\right)\right\} \cos \left(2 E^{\prime}+2 g+2 h\right) \\
& \left.\left.-e^{\prime}\left\{\left(2-e^{\prime 2}\right)+2\left(1-e^{\prime 2}\right)^{1 / 2}\right\} \cos \left(3 E^{\prime}+2 g+2 h\right)\right]\right\} \frac{a^{\prime}}{r^{\prime}} \sin E^{\prime}, \\
& \frac{\partial S_{2}}{\partial g}=\frac{1}{4} \frac{n^{\prime} R_{\mathbb{C}}^{2} J_{2}}{\left(1-e^{\prime 2}\right)^{3 / 2}} \sin ^{2} I^{\prime}\left[3 e^{\prime} \cos \left(f^{\prime}+2 g\right)+3 \cos \left(2 f^{\prime}+2 g\right)+e^{\prime} \cos \left(3 f^{\prime}+2 g\right)\right] \\
& +\frac{1}{4} \frac{n^{\prime} R_{C}^{2} J_{22}}{\left(1-e^{\prime 2}\right)^{3 / 2}}\left\{\left(1-\cos I^{\prime}\right)^{2}\left[3 e^{\prime} \cos \left(f^{\prime}+2 g-2 h\right)+3 \cos \left(2 f^{\prime}+2 g-2 h\right)+e^{\prime} \cos \left(3 f^{\prime}+2 g-2 h\right)\right]\right. \\
& \left.+\left(1+\cos I^{\prime}\right)^{2}\left[3 e^{\prime} \cos \left(f^{\prime}+2 g+2 h\right)+3 \cos \left(2 f^{\prime}+2 g+2 h\right)+e^{\prime} \cos \left(3 f^{\prime}+2 g+2 h\right)\right]\right\} \\
& -\frac{1}{32} \frac{n^{\prime} R_{C}^{3} J_{3} \sin I}{a^{\prime}\left(1-e^{\prime 2}\right)^{5 / 2}}\left\{\left\{( 1 - 5 \operatorname { c o s } ^ { 2 } I ^ { \prime } ) \left[12 e^{\prime}\left(f^{\prime}-l\right) \cos g+3 e^{\prime 2} \sin \left(f^{\prime}-g\right)+6\left(2+e^{\prime 2}\right) \sin \left(f^{\prime}+g\right)\right.\right.\right. \\
& \left.+6 e^{\prime} \sin \left(2 f^{\prime}+g\right)+e^{\prime 2} \sin \left(3 f^{\prime}+g\right)\right]-\sin ^{2} I^{\prime}\left[15 e^{\prime 2} \sin \left(f^{\prime}+3 g\right)+30 e^{\prime} \sin \left(2 f^{\prime}+3 g\right)\right. \\
& \left.\left.+10\left(2+e^{\prime 2}\right) \sin \left(3 f^{\prime}+3 g\right)+15 e^{\prime} \sin \left(4 f^{\prime}+3 g\right)+3 e^{\prime 2} \sin \left(5 f^{\prime}+3 g\right)\right]\right\} \\
& \left.+\frac{1}{128} \frac{n^{\prime} R}{a^{\prime 2}\left(1-e^{\prime} J_{4} \sin ^{2} I\right.}\right)^{7 / 2}\left\{( 1 - 7 \operatorname { c o s } ^ { 2 } I ^ { \prime } ) \left[-60 e^{\prime 2}\left(f^{\prime}-l\right) \sin 2 g-10 e^{\prime 3} \cos \left(f^{\prime}-2 g\right)+30 e^{\prime}\left(4+e^{\prime 2}\right) \cos \left(f^{\prime}+2 g\right)\right.\right. \\
& \left.+20\left(2+3 e^{\prime 2}\right) \cos \left(2 f^{\prime}+2 g\right)+10 e^{\prime}\left(4+e^{\prime 2}\right) \cos \left(3 f^{\prime}+2 g\right)+15 e^{\prime 2} \cos \left(4 f^{\prime}+2 g\right)+2 e^{\prime 3} \cos \left(5 f^{\prime}+2 g\right)\right] \\
& -\sin ^{2} I^{\prime}\left[35 e^{\prime 3} \cos \left(f^{\prime}+4 g\right)+105 e^{\prime 2} \cos \left(2 f^{\prime}+4 g\right)+35 e^{\prime}\left(4+e^{\prime 2}\right) \cos \left(3 f^{\prime}+4 g\right)\right.
\end{aligned}
$$




$$
\begin{aligned}
& \left.\left.+35\left(2+3 e^{\prime 2}\right) \cos \left(4 f^{\prime}+4 g\right)+21 e^{\prime}\left(4+e^{\prime 2}\right) \cos \left(5 f^{\prime}+4 g\right)+35 e^{\prime 2} \cos \left(6 f^{\prime}+4 g\right)+5 e^{\prime 3} \cos \left(7 f^{\prime}+4 g\right)\right]\right\} \\
& -\frac{1}{2048} \mathrm{n}^{\prime} \mathrm{R}_{\mathbb{C}}^{5} \mathrm{~J}_{5} \frac{\sin I^{\prime}}{\mathrm{a}^{3}\left(1-\mathrm{e}^{\prime 2}\right)^{9 / 2}}\left\{2 ( 1 - 1 4 \operatorname { c o s } ^ { 2 } I ^ { \prime } + 2 1 \operatorname { c o s } ^ { 4 } I ^ { \prime } ) \left[120 \mathrm{e}^{\prime}\left(4+3 \mathrm{e}^{\prime 2}\right)\left(\mathrm{f}^{\prime}-l\right) \cos \mathrm{g}\right.\right. \\
& +30\left(8+24 e^{\prime 2}+3 e^{\prime 4}\right) \sin \left(f^{\prime}+g\right)+60 e^{\prime 2}\left(6+e^{\prime 2}\right) \sin \left(f^{\prime}-g\right)+60 e^{\prime}\left(4+3 e^{\prime 2}\right) \sin \left(2 f^{\prime}+g\right) \\
& +60 e^{\prime 3} \sin \left(2 f^{\prime}-g\right)+20 e^{\prime 2}\left(6+e^{\prime 2}\right) \sin \left(3 f^{\prime}+g\right)+5 e^{\prime 4} \sin \left(3 f^{\prime}-g\right)+30 e^{\prime 3} \sin \left(4 f^{\prime}+g\right) \\
& \left.+3 e^{\prime 4} \sin \left(5 f^{\prime}+g\right)\right]-\sin ^{2} I^{\prime}\left(1-9 \cos ^{2} I^{\prime}\right)\left[840 e^{\prime 3}\left(f^{\prime}-l\right) \cos 3 g+420 e^{\prime 2}\left(6+e^{\prime 2}\right) \sin \left(f^{\prime}+3 g\right)\right. \\
& +105 e^{\prime 4} \sin \left(f^{\prime}-3 g\right)+420 e^{\prime}\left(4+3 e^{\prime 2}\right) \sin \left(2 f^{\prime}+3 g\right)+70\left(8+24 e^{\prime 2}+3 e^{\prime 4}\right) \sin \left(3 f^{\prime}+3 g\right) \\
& +210 e^{\prime}\left(4+3 e^{\prime 2}\right) \sin \left(4 f^{\prime}+3 g\right)+84 e^{\prime 2}\left(6+e^{\prime 2}\right) \sin \left(5 f^{\prime}+3 g\right)+140 e^{\prime 3} \sin \left(6 f^{\prime}+3 g\right) \\
& \left.15 e^{\prime 4} \sin \left(7 f^{\prime}+3 g\right)\right]+\sin ^{4} I^{\prime}\left[315 e^{\prime 4} \sin \left(f^{\prime}+5 g\right)+1260 e^{\prime 3} \sin \left(2 f^{\prime}+5 g\right)\right. \\
& +420 e^{\prime 2}\left(6+e^{\prime 2}\right) \sin \left(3 f^{\prime}+5 g\right)+630 e^{\prime}\left(4+3 e^{\prime 2}\right) \sin \left(4 f^{\prime}+5 g\right)+126\left(8+24 e^{\prime 2}+3 e^{\prime 4}\right) \sin \left(5 f^{\prime}+5 g\right) \\
& +420 e^{\prime}\left(4+3 e^{\prime 2}\right) \sin \left(6 f^{\prime}+5 g\right)+180 e^{\prime 2}\left(6+e^{\prime 2}\right) \sin \left(7 f^{\prime}+5 g\right)+315 e^{\prime 3} \sin \left(8 f^{\prime}+5 g\right) \\
& \left.\left.+35 e^{\prime 4} \sin \left(9 f^{\prime}+5 g\right)\right]\right\} \\
& +\frac{1}{64} \frac{n^{\prime} a^{\prime 2}}{\epsilon}\left(\frac{n_{\overparen{C}}}{n^{\prime}}\right)^{2}\left\{-60 e^{\prime 2}\left[2 \sin ^{2} I^{\prime} \sin 2 g+\left(1-\cos I^{\prime}\right)^{2} \sin (2 g-2 h)\right.\right. \\
& \left.+\left(1+\cos I^{\prime}\right)^{2} \sin (2 g+2 h)\right]\left(E^{\prime}-l\right)+2 \sin ^{2} I^{\prime}\left[1 5 e ^ { \prime } \left\{\left(2+e^{\prime 2}\right)\right.\right. \\
& \left.-2\left(1-e^{\prime 2}\right)^{1 / 2}\right\} \cos \left(E^{\prime}-2 g\right)-3\left\{\left(2+e^{\prime 2}\right)-2\left(1-e^{\prime 2}\right)^{1 / 2}\left(1+e^{\prime 2}\right)\right\} \cos \left(2 E^{\prime}-2 g\right) \\
& +e^{\prime}\left\{\left(2-e^{\prime} \dot{2}\right)-2\left(1-e^{\prime 2}\right)^{1 / 2}\right\} \cos \left(3 E^{\prime}-2 g\right) \\
& -15 e^{\prime}\left\{\left(2+e^{\prime 2}\right)+2\left(1-e^{\prime 2}\right)^{1 / 2}\right\} \cos \left(E^{\prime}+2 g\right) \\
& +3\left\{\left(2+e^{\prime 2}\right)+2\left(1-e^{\prime 2}\right)^{1 / 2}\left(1+e^{\prime 2}\right)\right\} \cos \left(2 E^{\prime}+2 g\right) \\
& \left.-e^{\prime}\left\{\left(2-e^{\prime 2}\right)+2\left(1-e^{\prime 2}\right)^{1 / 2}\right\} \cos \left(3 E^{\prime}+2 g\right)\right]
\end{aligned}
$$




$$
\begin{aligned}
& +\left(1-\cos I^{\prime}\right)^{2}\left[15 e^{\prime}\left\{\left(2+e^{\prime 2}\right)-2\left(1-e^{\prime 2}\right)^{1 / 2}\right\} \cos \left(E^{\prime}-2 g+2 h\right)\right. \\
& -3\left\{\left(2+e^{\prime 2}\right)-2\left(1-e^{\prime 2}\right)^{1 / 2}\left(1+e^{\prime 2}\right)\right\} \cos \left(2 E^{\prime}-2 g^{\prime}+2 h\right) \\
& +e^{\prime}\left\{\left(2-e^{\prime 2}\right)-2\left(1-e^{\prime 2}\right)^{1 / 2}\right\} \cos \left(3 E^{\prime}-2 g+2 h\right) \\
& -15 e^{\prime}\left\{\left(2+e^{\prime 2}\right)+2\left(1-e^{\prime 2}\right)^{1 / 2}\right\} \cos \left(E^{\prime}+2 g-2 h\right) \\
& +3\left\{\left(2+e^{\prime 2}\right)+2\left(1-e^{\prime 2}\right)^{1 / 2}\left(1+e^{\prime 2}\right)\right\} \cos \left(2 E^{\prime}+2 g-2 h\right) \\
& \left.-e^{\prime}\left\{\left(2-e^{\prime 2}\right)+2\left(1-e^{\prime 2}\right)^{1 / 2}\right\} \cos \left(3 E^{\prime}+2 g-2 h\right)\right] \\
& +\left(1+\cos I^{\prime}\right)^{2}\left[15 e^{\prime}\left\{\left(2+e^{\prime 2}\right)-2\left(1-e^{\prime 2}\right)^{1 / 2}\right\} \cos \left(E^{\prime}-2 g-2 h\right)\right. \\
& -3\left\{\left(2+e^{\prime 2}\right)-2\left(1-e^{\prime 2}\right)^{1 / 2}\left(1+e^{\prime 2}\right)\right\} \cos \left(2 E^{\prime}-2 g-2 h\right) \\
& +3\left\{\left(2+e^{\prime 2}\right)+2\left(1-e^{\prime 2}\right)^{1 / 2}\left(1+e^{\prime 2}\right)\right\} \cos \left(2 E^{\prime}+2 g+2 h\right) \\
& -15 e^{\prime}\left\{\left(2+e^{\prime 2}\right)+2\left(1-e^{\prime 2}\right)^{1 / 2}\right\} \cos \left(E^{\prime}+2 g+2 h\right) \\
& \left.+\left(2-e^{\prime 2}\right)-2\left(1-e^{\prime 2}\right)^{1 / 2}\right\} \cos \left(3 E^{\prime}-2 g-2 h\right)
\end{aligned}
$$

and

$$
\begin{aligned}
& \frac{\partial S_{2}}{\partial h}=\frac{1}{4} \frac{n^{\prime} R_{\mathbb{C}}^{2} J_{22}}{\left(1-e^{\prime 2}\right)^{-3 / 2}}\left\{6 \sin ^{2} I^{\prime}\left[-2\left(f^{\prime}-l\right) \sin 2 h-e^{\prime} \cos \left(f^{\prime}-2 h\right)+e^{\prime} \cos \left(f^{\prime}+2 h\right)\right]\right. \\
& -\left(1-\cos I^{\prime}\right)^{2}\left[3 e^{\prime} \cos \left(f^{\prime}+2 g-2 h\right)+3 \cos \left(2 f^{\prime}+2 g-2 h\right)+e^{\prime} \cos \left(3 f^{\prime}+2 g-2 h\right)\right] \\
& \left.+\left(1+\cos I^{\prime}\right)^{2}\left[3 e^{\prime} \cos \left(f^{\prime}+2 g+2 h\right)+3 \cos \left(2 f^{\prime}+2 g+2 h\right)+e^{\prime} \cos \left(3 f^{\prime}+2 g+2 h\right)\right]\right\} \\
& +\frac{1}{192} \frac{n^{\prime} a^{\prime 2}}{\epsilon}\left(\frac{n_{\overparen{C}}}{n^{\prime}}\right)^{2}\left\{3 6 \left[-2 \sin ^{2} I^{\prime}\left(2+3 e^{\prime 2}\right) \sin 2 h+5 e^{\prime 2}\left(1-\cos I^{\prime}\right)^{2} \sin (2 g-2 h)\right.\right.
\end{aligned}
$$




$$
\begin{aligned}
& \left.-5 e^{\prime 2}\left(1+\cos I^{\prime}\right)^{2} \sin (2 g+2 h)\right]\left(E^{\prime}-l\right) \\
& +6 \sin ^{2} I^{\prime}\left[9 e^{\prime}\left(4+e^{\prime 2}\right) \cos \left(E^{\prime}-2 h\right)-9 e^{\prime 2} \cos \left(2 E^{\prime}-2 h\right)+e^{\prime 3} \cos \left(3 E^{\prime}-2 h\right)\right. \\
& \left.-9 e^{\prime}\left(4+e^{\prime 2}\right) \cos \left(E^{\prime}+2 h\right)+9 e^{\prime 2} \cos \left(2 E^{\prime}+2 h\right)-e^{\prime 3} \cos \left(3 E^{\prime}+2 h\right)\right] \\
& +3\left(1-\cos I^{\prime}\right)^{2}\left[-15 e^{\prime}\left\{\left(2+e^{\prime 2}\right)-2\left(1-e^{\prime 2}\right)^{1 / 2}\right\} \cos \left(E^{\prime}-2 g+2 h\right)\right. \\
& +3\left\{\left(2+e^{\prime 2}\right)-2\left(1-e^{\prime 2}\right)^{1 / 2}\left(1+e^{\prime 2}\right)\right\} \cos \left(2 E^{\prime}-2 g+2 h\right) \\
& -e^{\prime}\left\{\left(2-e^{\prime 2}\right)-2\left(1-e^{\prime 2}\right)^{1 / 2}\right\} \cos \left(3 E^{\prime}-2 g+2 h\right) \\
& +15 e^{\prime}\left\{\left(2+e^{\prime 2}\right)+2\left(1-e^{\prime 2}\right)^{1 / 2}\right\} \cos \left(E^{\prime}+2 g-2 h\right) \\
& -3\left\{\left(2+e^{\prime 2}\right)+2\left(1-e^{\prime 2}\right)^{1 / 2}\left(1+e^{\prime 2}\right)\right\} \cos \left(2 E^{\prime}+2 g-2 h\right) \\
& \left.+e^{\prime}\left\{\left(2-e^{\prime 2}\right)+2\left(1-e^{\prime 2}\right)^{1 / 2}\right\} \cos \left(3 E^{\prime}+2 g-2 h\right)\right] \\
& +3\left(1+\cos I^{\prime}\right)^{2}\left[15 e^{\prime}\left\{\left(2+e^{\prime 2}\right)-2\left(1-e^{\prime 2}\right)^{1 / 2}\right\} \cos \left(E^{\prime}-2 g-2 h\right)\right. \\
& -3\left\{\left(2+e^{\prime 2}\right)-2\left(1-e^{\prime 2}\right)^{1 / 2}\left(1+e^{\prime 2}\right)\right\} \cos \left(2 E^{\prime}-2 g-2 h\right) \\
& +e^{\prime}\left\{\left(2-e^{\prime 2}\right)-2\left(1-e^{\prime 2}\right)^{1 / 2}\right\} \cos \left(3 E^{\prime}-2 g-2 h\right) \\
& -15 e^{\prime}\left\{\left(2+e^{\prime 2}\right)+2\left(1-e^{\prime 2}\right)^{1 / 2}\right\} \cos \left(E^{\prime}+2 g+2 h\right) \\
& +3\left\{\left(2+e^{\prime 2}\right)+2\left(1-e^{\prime 2}\right)^{1 / 2}\left(1+e^{\prime 2}\right)\right\} \cos \left(2 E^{\prime}+2 g+2 h\right) \\
& \left.\left.-\mathrm{e}^{\prime}\left\{\left(2-\mathrm{e}^{\prime 2}\right)+2\left(1-\mathrm{e}^{\prime 2}\right)^{1 / 2}\right\} \cos \left(3 \mathrm{E}^{\prime}+2 \mathrm{~g}+2 \mathrm{~h}\right)\right]\right\} \text {. }
\end{aligned}
$$

If

$$
\begin{aligned}
& \Delta \mathrm{L}=\mathrm{L}-\mathrm{L}^{\prime}, \\
& \Delta \mathrm{G}=\mathrm{G}-\mathrm{G}^{\prime},
\end{aligned}
$$

and

$$
\Delta \mathbf{H}=\mathbf{H}-\mathbf{H}^{\prime},
$$


the short-period perturbations in the Keplerian elements a, e, I are obtained by

$$
\begin{aligned}
& a=a^{\prime}+2 a^{\prime} \frac{\Delta L}{L^{\prime}}, \\
& e=e^{\prime}+\frac{1-e^{\prime 2}}{e^{\prime}}\left(\frac{\Delta L}{L^{\prime}}-\frac{\Delta G}{G^{\prime}}\right)
\end{aligned}
$$

and

$$
I=I^{\prime}+\cot I^{\prime}\left(\frac{\Delta G}{G^{\prime}}-\frac{\Delta H}{H^{\prime}}\right),
$$

and, in the expressions for $\Delta \mathrm{L}, \Delta \mathrm{G}, \Delta \mathrm{H}$, the variables $\mathrm{l}, \mathrm{g}, \mathrm{h}$ may be replaced by the variables $l^{\prime}$, $\mathrm{g}^{\prime}, \mathrm{h}^{\prime}$ with an error of the fourth order.

\section{THE LONG-PERIOD TERMS; ELIMINATION OF $h^{\prime}$}

At this stage, the Hamiltonian $F^{\prime}=F_{0}^{\prime}+F_{1}^{\prime}+F_{2}^{\prime}$ depends on the variables $g^{\prime}, h^{\prime}, L^{\prime}, G^{\prime}$, and $\mathrm{H}^{\prime}$; and $\mathrm{L}^{\prime}$ is a constant with respect to time. The next step in von Zeipel's method consists of the elimination of $h^{\prime}$ and $g^{\prime}$. The elimination of $h^{\prime}$ is performed by means of a generating function

$$
S^{\prime}=L^{\prime} l^{\prime}+G^{\prime \prime} g^{\prime}+H^{\prime} h^{\prime}+S_{1}^{\prime}+S_{2}^{\prime}+\cdots
$$

of a canonical transformation from $\left(l^{\prime}, \mathrm{g}^{\prime}, \mathrm{h}^{\prime}, \mathrm{L}^{\prime}, \mathrm{G}^{\prime}, \mathrm{H}^{\prime}\right)$ to $\left(l^{\prime \prime}, \mathrm{g}^{\prime \prime}, \mathrm{h}^{\prime \prime}, \mathrm{L}^{\prime \prime}, \mathrm{G}^{\prime \prime}, \mathrm{H}^{\prime \prime}\right)$. The new Hamiltonian

$$
F=F_{0}^{\prime}+F_{1}+F_{2}^{\prime}+\cdots
$$

should be independent of $h^{\prime \prime}$.

In order to simplify the formulas, the following symbols are introduced:

$$
\begin{aligned}
\nu & \equiv \mu_{\mathbb{C}}, b \equiv R_{\mathbb{C}}, \\
\eta^{\prime} & =\frac{G^{\prime}}{L^{\prime}}-\sqrt{1-e^{\prime 2}} .
\end{aligned}
$$


and

$$
\theta^{\prime}=\frac{\mathrm{H}^{\prime}}{\mathrm{G}^{\prime}}=\cos \mathrm{I}^{\prime}
$$

Then, from Equation 39,

$$
\begin{aligned}
& F^{\prime}=\frac{\mu^{2}}{2 L^{\prime 2}}+n_{\mathbb{C}}^{*} H^{\prime}+\frac{n_{\mathbb{C}^{2} L^{\prime 4}}}{16 \mu^{2} \epsilon}\left\{\left(5-3 \eta^{\prime 2}\right)\left[\left(-1+3 \theta^{\prime 2}\right)+3\left(1-\theta^{\prime 2}\right) \cos 2 h^{\prime}\right]\right. \\
& \left.+15\left(1-\eta^{\prime 2}\right)\left[\frac{1}{2}\left(1+\theta^{\prime}\right)^{2} \cos 2\left(g^{\prime}+h^{\prime}\right)+\left(1-\theta^{\prime 2}\right) \cos 2 g^{\prime}+\frac{1}{2}\left(1-\theta^{\prime}\right)^{2} \cos 2\left(g^{\prime}-h^{\prime}\right)\right]\right\} \\
& +\frac{1}{4} \frac{u^{4}}{L^{\prime 6} \eta^{\prime 3}}\left[-b^{2} J_{2}\left(1-3 t^{\prime 2}\right)+6 b^{2} J_{22}\left(1-t^{\prime 2}\right) \cos 2 h^{\prime}\right] \\
& -\frac{3}{8} \frac{L^{5} b^{3} J_{3}}{L} \cdot \frac{\eta^{\prime}}{\eta^{\prime}}\left(1-\eta^{\prime 2}\right)^{1 / 2}\left(1-r^{\prime 2}\right)^{1 / 2}\left(1-5 c^{\prime 2}\right) \sin g^{\prime} \\
& -\frac{3}{128} \underset{L^{6}}{\mu^{\prime}} \frac{\mathrm{b}^{4} \mathrm{~J}_{4}}{\eta^{\prime 7}}\left[\left(5-3 \eta^{\prime 2}\right)\left(3-30 H^{\prime 2}+35^{\prime} t^{4}\right)-10\left(1-\eta^{\prime 2}\right)\left(1-t^{\prime 2}\right)\left(1-7 t^{\prime 2}\right) \cos 2 \mathrm{~g}^{\prime}\right]
\end{aligned}
$$

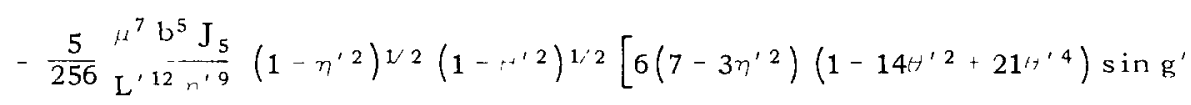

$$
\begin{aligned}
& \left.7\left(1-n^{\prime 2}\right)\left(1, \prime^{\prime \prime 2}\right)\left(1 \cdots 9 \prime^{\prime 2}\right) \sin 3 g^{\prime}\right] \text {. }
\end{aligned}
$$

The elimination of $h^{\prime}$ depends on the solution of the system

$$
\begin{aligned}
F_{0}^{\prime \prime}\left(L^{\prime \prime}\right) & =F_{0}^{\prime}\left(L^{\prime \prime}\right)=\frac{\mu^{2}}{2 L^{\prime 2}}, \\
F_{1}^{\prime \prime}\left(H^{\prime \prime}\right) & =F_{1}^{\prime}\left(H^{\prime \prime}\right)=n_{\mathbb{C}}^{*} H^{\prime \prime}, \\
F_{2}^{\prime \prime} & =F_{2}^{\prime}+\frac{\partial S_{1}^{\prime}}{\partial h^{\prime}} \frac{\partial F_{1}^{\prime}}{\partial H^{\prime \prime}},
\end{aligned}
$$

and, since $S_{1}^{\prime}$ does not depend on $l^{\prime}$,

$$
L^{\prime \prime}=L^{\prime} \text {. }
$$


As before, a particular solution is given by

$$
F_{2}^{\prime \prime}=F_{2 s}^{\prime}=\text { part of } F_{2}^{\prime} \text { independent of } h^{\prime}
$$

and

$$
\mathrm{n}_{\mathbb{C}}^{*} \frac{\partial \mathrm{S}_{1}^{\prime}}{\partial \mathrm{h}^{\prime}}=-\mathrm{F}_{2 \mathrm{p}}^{\prime}=-\left(\mathrm{F}_{2}^{\prime}-\mathbf{F}_{2 \mathrm{~s}}^{\prime}\right) .
$$

Then it is found that

$$
\begin{aligned}
& \mathrm{F}_{2}^{\prime \prime}=\frac{\mathrm{n}_{\mathbb{C}}^{2} \mathrm{~L}^{\prime 4}}{16 \mu^{2} \epsilon}\left[\left(5-3 \eta^{\prime \prime 2}\right)\left(-1+3 \theta^{\prime \prime 2}\right)\right. \\
& \left.+15\left(1-\eta^{\prime \prime 2}\right)\left(1-\theta^{\prime \prime 2}\right) \cos 2 g^{\prime \prime}\right]-\frac{1}{4} b^{2} J_{2} \frac{\mu^{4}}{L^{\prime 6}} \frac{1}{\eta^{\prime \prime}}\left(1-3 \theta^{\prime \prime 2}\right) \\
& -\frac{3}{8} b^{3} J_{3} \frac{\mu^{5}}{L^{\prime 8} \eta^{\prime \prime}}\left(1-\eta^{\prime 2}\right)^{1 / 2}\left(1-\tau^{\prime \prime 2}\right)^{1 / 2}(1-5 \cdot \cdot 2) \sin g \\
& -\frac{3}{128} b^{4} J_{4} \frac{\mu^{6}}{L^{\prime 10} \eta^{\prime}}\left[\left(5-3 \eta^{\prime \prime}\right)\left(3-30 t^{\prime \prime 2}+35^{\prime 4}\right)-10\left(1-\eta^{\prime \prime 2}\right)\left(1-\theta^{\prime \prime 2}\right)\left(1-7 \theta^{\prime \prime 2}\right) \cos 2 g^{\prime \prime}\right] \\
& -\frac{5}{256} \mathrm{~b}^{5} \mathrm{~J}_{5} \frac{\mu^{7}}{\mathrm{~L}^{\prime 12} \eta^{\prime \prime}}\left(1-\eta^{\prime 2}\right)^{1^{\prime 2}}\left(1-\eta^{\prime 2}\right)^{1 / 2}\left[6\left(7-3 \gamma^{\prime}{ }^{2}\right)\left(1-144^{\prime 2}+21{ }^{4}\right) \sin \mathrm{g}\right. \\
& \left.-7\left(1-\eta^{\prime \prime 2}\right)\left(1-\theta^{\prime \prime 2}\right)(1-9-2) \sin 3 g^{\prime \prime}\right] \text {. }
\end{aligned}
$$

where

$$
\eta^{\prime \prime}=\frac{\mathrm{G}^{\prime \prime}}{\mathrm{L}^{\prime}}
$$

and

$$
\theta^{\prime \prime}=\frac{\mathrm{H}^{\prime \prime}}{\mathrm{G}^{\prime \prime}} \text {. }
$$




\section{Furthermore,}

$$
\begin{aligned}
\mathrm{n}_{\mathbb{C}}^{*} \mathrm{~S}_{1}^{\prime}= & -\frac{1}{16} \frac{\mu^{2}}{\in \mathrm{L}^{\prime 2}}\left(\frac{\mathrm{n}_{\mathbb{C}}}{\mathrm{n}^{\prime}}\right)^{2}\left\{\frac{3}{2}\left(5-3 \eta^{\prime \prime}\right)\left(1-\theta^{\prime \prime} 2\right) \sin 2 \mathrm{~h}^{\prime}\right. \\
& \left.+\frac{15}{4}\left(1-\eta^{\prime \prime 2}\right)\left[\left(1+\theta^{\prime \prime}\right)^{2} \sin 2\left(g^{\prime}+h^{\prime}\right)-\left(1-\theta^{\prime \prime}\right)^{2} \sin 2\left(g^{\prime}-\mathrm{h}^{\prime}\right)\right]\right\} \\
& -\frac{3}{4} \mathrm{n}^{\prime 2} \mathrm{~b}^{2} \mathrm{~J}_{22} \frac{1}{\eta^{\prime \prime 3}}\left(1-\theta^{\prime \prime} 2\right) \sin 2 \mathrm{~h}^{\prime} .
\end{aligned}
$$

The long-period perturbations depending on the motion of the node are given by

$$
\begin{aligned}
L^{\prime} & =L^{\prime \prime}+\frac{\partial S_{1}^{\prime}}{\partial l^{\prime}}=L^{\prime \prime}, \\
G^{\prime} & G^{\prime}+\frac{\partial S_{1}^{\prime}}{\partial g^{\prime}} \\
& =G^{\prime \prime}\left\{1-\frac{15}{32 \epsilon}\left(\frac{n_{\mathbb{C}}^{2}}{n_{\mathbb{C}}^{*} n^{\prime}}\right) \frac{1-\eta^{\prime \prime 2}}{\eta^{\prime \prime}}\left[\left(1+\mu^{\prime \prime}\right)^{2} \cos 2\left(g^{\prime \prime}+h^{\prime \prime}\right)-\left(1-t^{\prime}\right)^{2} \cos 2\left(g^{\prime \prime}-h^{\prime}\right)\right]\right\},
\end{aligned}
$$

$$
\begin{aligned}
& \mathrm{H}^{\prime}=\mathrm{H}^{\prime \prime}+\frac{\partial \mathrm{S}_{1}{ }^{\prime}}{\partial \mathrm{h}^{\prime}} \\
& =H^{\prime \prime}\left\{1 \frac { 3 } { 1 6 \epsilon } ( \frac { n _ { \mathscr { C } } ^ { 2 } } { n _ { \mathbb { C } } ^ { * } n ^ { \prime } } ) \frac { 1 } { \eta ^ { \prime } H ^ { \prime \prime } } \left[\left(5-3 \eta^{\prime \prime 2}\right)\left(1-t^{\prime \prime 2}\right) \cos 2 h^{\prime \prime}+\frac{5}{2}\left(1 \eta^{\prime \prime 2}\right)\left\{\left(1+\theta^{\prime}\right)^{2} \cos 2\left(g^{\prime \prime}+h^{\prime \prime}\right)\right.\right.\right. \\
& \left.\left.\left.+\left(1-\mu^{\prime \prime}\right)^{2} \cos 2\left(\mathrm{~g}^{\prime \prime}-\mathrm{h}^{\prime}\right)\right\}\right]-\frac{3}{2} \frac{\mathrm{n}^{\prime}}{\mathrm{H}^{\prime}}\left(\frac{\mathrm{n}^{\prime}}{\mathrm{n}_{\mathbb{C}}^{*}}\right) \mathrm{b}^{2} J_{22} \frac{1-\mu^{-2}}{\eta^{\prime \prime}} \cos 2 h^{\prime \prime}\right\},
\end{aligned}
$$

$l^{\prime}=l^{\prime \prime}-\frac{\partial \mathrm{S}_{1}^{\prime}}{\partial \mathrm{L}}$

$$
\begin{aligned}
=l^{\prime \prime}+\frac{1}{16 \epsilon}\left(\frac{\mathrm{n}_{\mathscr{C}}^{2}}{n_{\mathbb{C}}^{*} \mathrm{n}^{\prime}}\right)\left\{3\left(10-3 \eta^{\prime \prime}\right)\right. & \left(1-\theta^{\prime \prime 2}\right) \sin 2 \mathrm{~h}^{\prime \prime}+15\left(1-\frac{1}{2} \eta^{\prime \prime 2}\right)\left[\left(1+\theta^{\prime \prime}\right)^{2} \sin 2\left(\mathrm{~g}^{\prime \prime}+\mathrm{h}^{\prime \prime}\right)\right. \\
& \left.\left.-\left(1-\theta^{\prime \prime}\right)^{2} \sin 2\left(\mathrm{~g}^{\prime \prime}-\mathrm{h}^{\prime \prime}\right)\right]\right\}-\frac{9}{4} \frac{\mathrm{n}^{\prime 2}}{\mathrm{n}_{\mathbb{C}}^{*}} \mathrm{~b}^{2} \mathrm{~J}_{22} \frac{1-\theta^{\prime \prime}}{\eta^{\prime 3} \mathrm{~L}^{\prime}} \sin 2 \mathrm{~h}^{\prime \prime},
\end{aligned}
$$




$$
\begin{aligned}
& \mathbf{g}^{\prime}= \mathrm{g}^{\prime \prime}-\frac{\partial \mathrm{S}_{1}^{\prime}}{\partial \mathrm{G}^{\prime \prime}} \\
&=\mathrm{g}^{\prime \prime}+\frac{1}{16 \epsilon}\left(\frac{\mathrm{n}_{\mathbb{C}}^{2}}{\mathrm{n}_{\mathbb{C}}^{*} \mathrm{n}^{\prime}}\right) \frac{1}{\eta^{\prime \prime}}\left[3\left(5 \theta^{\prime \prime 2}-3 \eta^{\prime \prime 2}\right) \sin 2 \mathrm{~h}^{\prime \prime}-\frac{15}{2}\left(1+\theta^{\prime \prime}\right)\left(\eta^{\prime \prime 2}+\theta^{\prime \prime}\right) \sin 2\left(\mathrm{~g}^{\prime \prime}+\mathrm{h}^{\prime \prime}\right)\right. \\
& \\
&\left.\quad+\frac{15}{2}\left(1-\theta^{\prime \prime}\right)\left(\eta^{\prime \prime 2}-\theta^{\prime \prime}\right) \sin 2\left(\mathrm{~g}^{\prime \prime}-\mathrm{h}^{\prime \prime}\right)\right]-\frac{3}{4} \frac{\mathrm{n}^{\prime 2}}{\mathrm{n}_{\mathbb{C}}^{*}} \mathrm{~b}^{2} \mathrm{~J}_{22} \frac{3-5 \theta^{\prime \prime 2}}{\mathrm{~L}^{\prime} \eta^{\prime \prime}} \sin 2 \mathrm{~h}^{\prime \prime},
\end{aligned}
$$

and

$$
\begin{aligned}
\mathrm{h}^{\prime}= & \mathrm{h}^{\prime \prime}-\frac{\partial \mathrm{S}_{1}^{\prime}}{\partial \mathrm{H}^{\prime \prime}} . \\
= & \mathrm{h}^{\prime \prime}+\frac{1}{16 \epsilon}\left(\frac{\mathrm{n}_{\mathbb{C}}^{2}}{\mathrm{n}_{\mathbb{C}}^{*} \mathrm{n}^{\prime}}\right) \frac{1}{\eta^{\prime \prime}}\left\{3 \theta^{\prime \prime}\left(3 \eta^{\prime \prime 2}-5\right) \sin 2 \mathrm{~h}^{\prime \prime}+\frac{15}{2}\left(1-\eta^{\prime \prime}\right)\left[\left(1+\theta^{\prime \prime}\right) \sin 2\left(\mathrm{~g}^{\prime \prime}+\mathrm{h}^{\prime \prime}\right)\right.\right. \\
& \left.\left.+\left(1-\theta^{\prime \prime}\right) \sin 2\left(\mathrm{~g}^{\prime \prime}-\mathrm{h}^{\prime \prime}\right)\right]\right\}-\frac{3}{2} \frac{\mathrm{n}^{\prime 2}}{\mathrm{n}_{\mathbb{C}}^{*}} \mathrm{~b}^{2} \mathrm{~J}_{22} \frac{\theta^{\prime \prime}}{\mathrm{L}^{\prime} \eta^{\prime \prime}} \sin 2 \mathrm{~h}^{\prime \prime} .
\end{aligned}
$$

It is important to note that these terms are factored by first-order factors. Then, a secondorder theory produces first-order long-period terms. It is necessary to go to third order to obtain second-order long-period terms. This is done next.

\section{THE SECOND-ORDER LONG-PERIOD TERMS AND ELIMINATION OF $h^{\prime}$ AND THE TIME}

The small parameters of third order are as follows:

$$
\begin{aligned}
& j_{2}\left(\frac{n_{\overparen{C}}}{n}\right)^{2} \quad \text { (earth's oblateness) } \\
& \left(\frac{n_{\oplus}}{n}\right)^{2} \quad \text { (sun's perturbation) } \\
& \left(\frac{n_{\overparen{C}}}{n}\right)^{2} \sin \frac{i_{\overparen{C}}}{2} \quad \begin{array}{c}
\text { (correction due to the inclination of } \\
\text { the moon's orbit to its equator) }
\end{array} \\
& \sigma \quad \text { (solar radiation) }
\end{aligned}
$$




$$
\begin{array}{ll}
\left(\frac{n_{\overparen{C}}}{n}\right)^{2} e_{\overparen{C}} & \text { (eccentricity of the moon's orbit) } \\
\left(\frac{n_{\overparen{C}}}{n}\right)^{3} & \begin{array}{c}
\text { (earth's perturbations -third } \\
\text { Legendre polynomial) }
\end{array}
\end{array}
$$

and

$$
\frac{a_{\odot}}{n} \quad \text { (correction due to physical libration) }
$$

Since short-period perturbations of the third order will be neglected, it is understood that all terms of third order which depend on $l$ will be ignored. Suppose that $F_{3}$ is the third-order part of the Hamiltonian and $\mathrm{F}_{3}{ }^{\prime}$ the part free of short-period terms. The Hamiltonian will be timedependent through the longitudes of the earth and of the sun. This fact introduces one more degree of freedom in the problem, and it is necessary to introduce the time as a canonical variable. This is done through use of the following equations $(r=\mathrm{t}+$ const.):

$$
\frac{\mathrm{d} r}{\mathrm{dt}}=-\frac{\partial j}{\partial \mathrm{T}}-1
$$

and

$$
\frac{\mathrm{dT}}{\mathrm{dt}} \cdot \frac{\partial \mathcal{J}}{\partial \tau}=\frac{\partial \mathcal{J}}{\partial \mathrm{t}}=\frac{\partial \mathbf{F}_{3}}{\partial \mathrm{t}} \cdot
$$

so that

$$
\text { is } F-T
$$

and

$$
T=-F_{3}+\text { const. }
$$

This additional complication does not affect the development of the theory up to this point, if one sets

$$
\frac{\partial S_{2}{ }^{\prime}}{\partial \tau}=0
$$

The next step is then to eliminate $h^{\prime}$ to third order to obtain second-order long-period perturbations depending on the argument $h^{\prime}$. 
The orders of magnitude are as follows:

$$
\begin{aligned}
& F_{0}^{\prime}=F_{0}^{\prime}-T=\frac{\mu^{2}}{2 L^{\prime}}-\mathrm{T} \\
& F_{1}^{\prime}=F_{1}^{\prime}=\mathrm{n}_{\mathbb{C}}^{*} \mathrm{H}^{\prime} \\
& F_{2}^{\prime}=F_{2}^{\prime}
\end{aligned}
$$

and

$$
F_{3}^{\prime}=F_{3}^{\prime},
$$

where $\mathrm{T}$ has been incorporated into $y_{0}^{\prime}$. The von Zeipel differential equation of the third order is then

$$
\mathcal{F}_{3}^{\prime}+\frac{\partial \mathrm{S}_{1}^{\prime}}{\partial \mathrm{h}^{\prime}} \frac{\partial_{2}^{F_{2}^{\prime}}}{\partial \mathrm{H}^{\prime \prime}}+\frac{\partial \mathrm{S}_{1}^{\prime}}{\partial \mathrm{g}^{\prime}} \frac{\partial^{\prime} \mathcal{F}_{2}^{\prime}}{\partial \mathrm{G}^{\prime \prime}}+\frac{\partial \mathrm{S}_{2}^{\prime}}{\partial \mathrm{h}^{\prime}} \frac{\partial \mathcal{F}_{1}^{\prime}}{\partial \mathrm{H}^{\prime \prime}}+\frac{\partial \mathrm{S}_{3}^{\prime}}{\partial \tau} \frac{\partial \mathcal{F}_{0}^{\prime}}{\partial \mathrm{T}}=\mathcal{F}_{3}^{\prime \prime}+\frac{\partial \mathrm{S}_{1}^{\prime}}{\partial \mathrm{G}^{\prime \prime}} \frac{\partial \mathcal{F}_{2}^{\prime \prime}}{\partial \mathrm{g}^{\prime}},
$$

or

$$
\mathrm{F}_{3}^{\prime}+\frac{\partial \mathrm{S}_{1}^{\prime}}{\partial \mathrm{h}^{\prime}} \frac{\partial \mathrm{F}_{2}^{\prime}}{\partial \mathrm{H}^{\prime \prime}}+\frac{\partial \mathrm{F}_{2}^{\prime}}{\partial \mathrm{G}^{\prime \prime}} \frac{\partial \mathrm{S}_{1}^{\prime}}{\partial \mathrm{g}^{\prime}}+\mathrm{n}_{\mathbb{C}}^{*} \frac{\partial \mathrm{S}_{2}^{\prime}}{\partial \mathrm{h}^{\prime}}-\frac{\partial \mathrm{S}_{3}^{\prime}}{\partial \tau}=\mathrm{F}_{3}^{\prime \prime}+\frac{\partial \mathrm{F}_{2}^{\prime \prime}}{\partial \mathrm{g}^{\prime}} \frac{\partial \mathrm{S}_{1}^{\prime}}{\partial \mathrm{G}^{\prime \prime}}
$$

Since we had supposed that $\partial \mathrm{S}_{2}^{\prime} / \partial \tau=0$ in the previous order evaluation, a particular solution of the third-order equation is found by taking

$$
\mathrm{F}_{3}^{\prime \prime}=\mathrm{F}_{3 \mathrm{~s}}^{\prime}+\left(\frac{\partial \mathrm{S}_{1}^{\prime}}{\partial \mathrm{h}^{\prime}} \frac{\partial \mathrm{F}_{2}^{\prime}}{\partial \mathrm{H}^{\prime \prime}}\right)_{\mathrm{s}}+\left(\frac{\partial \mathrm{F}_{2}^{\prime}}{\partial \mathrm{G}^{\prime \prime}} \frac{\partial \mathrm{S}_{1}^{\prime}}{\partial \mathrm{g}^{\prime}}\right)_{\mathrm{s}}-\left(\frac{\partial \mathrm{F}_{2}^{\prime \prime}}{\partial \mathrm{g}^{\prime}} \frac{\partial \mathrm{S}_{1}^{\prime}}{\partial \mathrm{G}^{\prime \prime}}\right)_{\mathrm{s}},
$$

where the subscript s designates non-dependence upon $h^{\prime \prime}$ and $\tau$. Further,

$$
\mathrm{n}_{\overparen{C}}^{*} \frac{\partial \mathrm{S}_{2}^{\prime}}{\partial \mathrm{h}^{\prime}}=-\mathrm{F}_{3 \mathrm{ph}^{\prime}}^{\prime}-\left(\frac{\partial \mathrm{S}_{1}^{\prime}}{\partial \mathrm{h}^{\prime}} \frac{\partial \mathrm{F}_{2}^{\prime}}{\partial \mathrm{H}^{\prime \prime}}\right)_{\mathrm{ph}},-\left(\frac{\partial \mathrm{F}_{2}^{\prime}}{\partial \mathrm{G}^{\prime \prime}} \frac{\partial \mathrm{S}_{1}^{\prime}}{\partial \mathrm{g}^{\prime}}\right)_{\mathrm{ph}^{\prime}}+\left(\frac{\partial \mathrm{F}_{2}^{\prime \prime}}{\partial \mathrm{g}^{\prime}} \frac{\partial \mathrm{S}_{1}^{\prime}}{\partial \mathrm{G}^{\prime \prime}}\right)_{\mathrm{ph}^{\prime}},
$$

and

$$
\frac{\partial \mathrm{S}_{3}^{\prime}}{\partial \tau}=\mathrm{F}_{3 \mathrm{p} \tau}^{\prime}+\left(\frac{\partial \mathrm{S}_{1}^{\prime}}{\partial \mathbf{h}^{\prime}} \frac{\partial \mathrm{F}_{2}{ }^{\prime}}{\partial \mathrm{H}^{\prime \prime}}\right)_{\mathbf{p} \tau}+\left(\frac{\partial \mathrm{F}_{2}^{\prime}}{\partial \mathrm{G}^{\prime \prime}} \frac{\partial \mathrm{S}_{1}{ }^{\prime}}{\partial \mathrm{g}^{\prime}}\right)_{\mathbf{p} \tau}-\left(\frac{\partial \mathrm{F}_{2}^{\prime \prime}}{\partial \mathrm{g}^{\prime}} \frac{\partial \mathrm{S}_{1}{ }^{\prime}}{\partial \mathrm{G}^{\prime}}\right)_{\mathbf{p} \tau},
$$


where the subscript $\mathrm{ph}^{\prime}$ indicates the inclusion of all trigonometric terms with arguments of the form $\mathrm{jh}^{\prime}+\mathrm{kg}^{\prime}$, where $\mathrm{j}$ and $\mathrm{k}$ are integers $(\mathrm{j} \neq 0)$, and the subscript $\mathrm{p} \tau$ indicates the inclusion of all trigonometric terms with arguments of the form $j \tau+\mathrm{kh}^{\prime}+\mathrm{mg}^{\prime}$, where $j, k$, and $\mathrm{m}$ are integers $(j \neq 0)$.

Then $S_{2}^{\prime}$ will be obtained as a periodic function independent of the time, and $S_{3}^{\prime}$ as a periodic function dependent on time.

Since the functions $F_{2 p}^{\prime}, F_{2}^{\prime}, S_{1}{ }^{\prime}$, and $F_{2}^{\prime \prime}$ are known, the only parts that have to be computed are those corresponding to the third-order terms of the Hamiltonian $\left(\mathrm{F}_{3}{ }^{\prime}\right)$.

\section{THIRD-ORDER TERMS GENERATED BY COUPLING OF SECOND-ORDER TERMS}

It was found (from Equations 55 and 58) that

$$
\begin{aligned}
& F_{2}^{\prime}=\frac{1}{16 \epsilon}\left(\frac{n_{\mathbb{C}}}{n^{\prime}}\right)^{2} n^{\prime} L^{\prime}\left\{\left(5-3 \eta^{\prime 2}\right)\left[\left(-1+3 \dot{H}^{\prime 2}\right)+3\left(1-\theta^{\prime 2}\right) \cos 2 h^{\prime}\right]\right. \\
& \left.+15\left(1-\eta^{\prime 2}\right)\left[\frac{1}{2}\left(1+\eta^{\prime}\right)^{2} \cos 2\left(g^{\prime}+h^{\prime}\right)+\left(1-H^{\prime 2}\right) \cos 2 g^{\prime}+\frac{1}{2}\left(1-\psi^{\prime}\right)^{2} \cos 2\left(g^{\prime}-h^{\prime}\right)\right]\right\} \\
& +\frac{1}{4} \frac{\mu^{4}}{L^{\prime 6} \eta^{\prime 3}}\left[-b^{2} J_{2}\left(1-3^{H^{\prime} 2}\right)+6 b^{2} J_{22}\left(1-H^{\prime 2}\right) \cos 2 h^{\prime}\right]-\frac{3}{8} \frac{\mu^{5} b^{3} J_{3}}{L^{\prime 8} \eta^{\prime 5}}\left(1-\eta^{\prime 2}\right)^{1 / 2}\left(1-\theta^{\prime 2}\right)^{1 / 2}\left(1-5 C^{\prime 2}\right) \sin g^{\prime} \\
& -\frac{3}{128} \frac{\iota^{6} b^{4} \mathrm{~J}_{4}}{\mathrm{~L}^{\prime}{ }^{10} \eta^{\prime 7}}\left[\left(5-3 \eta^{\prime 2}\right)\left(3-30 t^{\prime 2}+35 t^{\prime 4}\right)-10\left(1-\eta^{\prime 2}\right)\left(1-H^{\prime 2}\right)\left(1-7 \xi^{\prime 2}\right) \cos 2 g^{\prime}\right] \\
& -\frac{5}{256} \frac{u^{7} \mathrm{~b}^{5} \mathrm{~J}_{5}}{\mathrm{~L}^{\prime 12} \eta_{\eta^{\prime}}}\left(1-\eta^{\prime 2}\right)^{1 / 2}\left(1-\xi^{\prime 2}\right)^{1 / 2}\left[6\left(7-3 \eta^{\prime 2}\right)\left(1-14 e^{\prime 2}+21 \prime^{\prime 4}\right) \sin \mathrm{g}^{\prime}-7\left(1-\eta^{\prime 2}\right)\left(1-\xi^{\prime 2}\right)\left(1-9 \theta^{\prime 2}\right) \sin 3 \mathrm{~g}^{\prime}\right]
\end{aligned}
$$

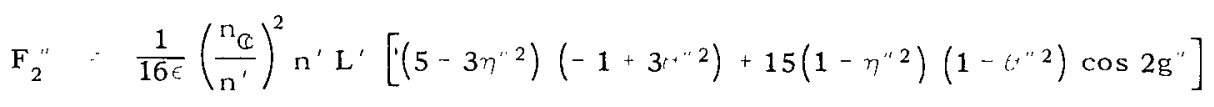

$$
\begin{aligned}
& -\frac{1}{4} b^{2} J_{2} \frac{\mu^{4}}{L^{\prime 6}} \frac{1}{\eta^{\prime \prime 3}}\left(1-3 \theta^{\prime \prime 2}\right)-\frac{3}{8} b^{3} J_{3} \frac{\mu^{5}}{L^{\prime 8} \eta^{\prime \prime}}\left(1-\eta^{\prime \prime 2}\right)^{1 / 2}\left(1-\theta^{\prime \prime 2}\right)^{1 / 2}\left(1-5 \theta^{\prime \prime 2}\right) \sin g^{\prime \prime} \\
& -\frac{3}{128} \mathrm{~b}^{4} \mathrm{~J}_{4} \frac{\mu^{6}}{\mathrm{~L}^{\prime 10} \eta^{\prime \prime}}\left[\left(5-3 \eta^{\prime \prime 2}\right)\left(3-30 \theta^{\prime \prime 2}+35 \theta^{\prime \prime 4}\right)-10\left(\mathrm{i}-\eta^{\prime \prime 2}\right)\left(1-\theta^{\prime \prime 2}\right)\left(1-7 \theta^{\prime \prime 2}\right) \cos 2 \mathrm{~g}^{\prime \prime}\right] \\
& -\frac{5}{256} \mathrm{~b}^{5} \mathrm{~J}_{5} \frac{\mu^{7}}{\mathrm{~L}^{\prime 12} \eta^{\prime \prime}}\left(1-\eta^{\prime \prime 2}\right)^{1 / 2}\left(1-\theta^{\prime \prime 2}\right)^{1 / 2}\left[6\left(7-3 \eta^{\prime \prime 2}\right)\left(1-14 \theta^{\prime \prime 2}+21 \theta^{\prime \prime 4}\right) \sin \mathrm{g}^{\prime \prime}\right. \\
& \left.-7\left(1-\eta^{\prime \prime}\right)\left(1-\theta^{\prime \prime 2}\right)\left(1-9 \theta^{\prime \prime 2}\right) \sin 3 g^{\prime \prime}\right] \text {, }
\end{aligned}
$$


and $\mathrm{n}_{\overparen{C}}^{*} \mathrm{~S}_{1}^{\prime}$ is given by Equation 59. In the coupling terms, the variables in $F_{2}^{\prime}$ can be double-primed with an error that is of the fourth order.

The partials needed are given below.

$$
\begin{aligned}
& \frac{\partial \mathbf{F}_{2}^{\prime}}{\partial \mathrm{H}^{\prime \prime}}=\frac{3}{16 \epsilon} \mathrm{n}^{\prime}\left(\frac{\mathrm{n}_{\mathbb{C}}}{\mathrm{n}^{\prime}}\right)^{2} \frac{1}{\eta^{\prime \prime} \theta^{\prime \prime}}\left\{2\left(5-3 \eta^{\prime \prime}\right) \theta^{\prime \prime} 2\left(1-\cos 2 \mathrm{~h}^{\prime \prime}\right)\right. \\
& +5\left(1-\eta^{\prime \prime}\right) \theta^{\prime \prime}\left[\left(1+\theta^{\prime \prime}\right) \cos 2\left(\mathrm{~g}^{\prime \prime}+\mathrm{h}^{\prime \prime}\right)-2 \theta^{\prime \prime} \cos 2 \mathrm{~g}^{\prime \prime}\right. \\
& \left.\left.-\left(1-\theta^{\prime \prime}\right) \cos 2\left(\mathrm{~g}^{\prime \prime}-\mathrm{h}^{\prime \prime}\right)\right]\right\}+\frac{3}{2} \mathrm{~b}^{2} \mathrm{n}^{\prime 2} \frac{\theta^{\prime \prime}}{\mathrm{L}^{\prime} \eta^{\prime \prime}}\left(\mathrm{J}_{2}-2 \mathrm{~J}_{22} \cos 2 \mathrm{~h}^{\prime \prime}\right) \\
& +\frac{3}{8} \mathrm{~b}^{3} \mathrm{~J}_{3} \frac{\mu \mathrm{n}^{\prime 2} \theta^{\prime \prime}\left(11-15 H^{\prime \prime 2}\right)\left(1-\eta^{\prime \prime}\right)^{1 / 2}}{\mathrm{~L}^{\prime 3} \eta^{\prime \prime}\left(1-f^{\prime \prime 2}\right)^{1 / 2}} \sin \mathrm{g}^{\prime \prime} \\
& +\frac{15}{32} \mathrm{~b}^{4} \mathrm{~J}_{4} \frac{\mu^{2} \mathrm{n}^{\prime 2} \mathrm{r}^{\prime \prime}}{\mathrm{L}^{\prime 5} \eta^{\prime \prime}}\left[\left(\left(5-3 \eta^{\prime \prime 2}\right)\left(3-7 r^{\prime} \cdot 2\right)-2\left(1-\eta^{\prime 2}\right)\left(4-7 r^{\prime \prime 2}\right) \cos 2 \mathrm{~g}\right]\right. \\
& +\frac{15}{256} \mathrm{~b}^{5} \mathrm{~J}_{5} \frac{\mu^{3} \mathrm{n}^{\prime 2}}{\mathrm{~L}^{\prime 7} \eta^{\prime \prime}}\left(1 0 ( 1 - \eta ^ { \prime \prime 2 } ) ^ { 1 / 2 } [ 2 ) ^ { 1 / 2 } \left[2\left(7-3 \eta^{\prime \prime 2}\right)\left(29-126^{\prime \prime 2}+105^{\prime \prime 4}\right) \sin \mathrm{g}\right.\right. \\
& \left.-7\left(1-\eta^{\prime \prime 2}\right)\left(1-\psi^{\prime \prime 2}\right)\left(7-15 r^{\prime \prime 2}\right) \sin 3 g^{\prime \prime}\right] \\
& \frac{\partial \mathrm{F}_{2}^{\prime}}{\partial \mathrm{G}^{\prime \prime}}-\frac{3 \mathrm{n}^{\prime}}{16 \epsilon}\left(\frac{\mathrm{n}_{\mathbb{C}}}{\mathrm{n}^{\prime}}\right)^{2} \frac{1}{\eta^{\prime \prime}}\left[2\left(\eta^{\prime \prime 2}-5{F^{\prime \prime 2}}^{\prime 2}\right)+2\left(5^{\prime 2}-3 \eta^{\prime \prime 2}\right) \cos 2 \mathrm{~h}^{\prime}\right. \\
& -5\left(1+\dot{U}^{\prime \prime}\right)\left(\eta^{\prime \prime 2}+\theta^{\prime \prime}\right) \cos 2\left(\mathrm{~g}^{\prime \prime}+\mathrm{h}^{\prime \prime}\right)+10\left(*^{\prime \prime 2}-r^{\prime \prime 2}\right) \cos 2 \mathrm{~g}^{\prime \prime} \\
& \left.\left.+5\left(1-\theta^{\prime \prime}\right)\left(\theta^{\prime \prime}-\eta^{\prime \prime 2}\right) \cos 2\left(\mathrm{~g}^{\prime \prime}-\mathrm{h}^{\prime \prime}\right)\right]+\frac{3}{4} \frac{\mathrm{n}^{\prime 2} \mathrm{~b}^{2}}{\mathrm{~L}^{\prime} \eta^{\prime \prime 4}}\left[\mathrm{~J}_{2}\left(1-5 \theta^{\prime \prime 2}\right)-2 \mathrm{~J}_{22}(3-5)^{\prime \prime 2}\right) \cos 2 \mathrm{~h}^{\prime \prime}\right] \\
& +\frac{3}{8} \mathrm{~b}^{3} \mathrm{~J}_{3} \overline{\mathrm{L}^{\prime 3} \eta^{\prime \prime}\left(1-\eta^{\prime \prime}\right)^{1 / 2}\left(1-\theta^{\prime \prime 2}\right)^{1 / 2}}\left[5-41 \theta^{\prime \prime 2}+40 \Theta^{\prime \prime 4}-\eta^{\prime \prime 2}\left(4-35 H^{\prime \prime 2}+35 \mathrm{e}^{\prime \prime 4}\right)\right] \sin \mathrm{g}^{\prime \prime} \\
& +\frac{15}{128} b^{4} J_{4} \frac{\mu^{2} n^{\prime 2}}{L^{\prime 5} \eta^{\prime \prime}}\left\{21-270 t^{\prime \prime 2}+385 r^{\prime \prime 4}-9 \eta^{\prime \prime 2}\left(1-14 r^{\prime \prime 2}+21^{\prime \prime \prime} 4\right)\right. \\
& \left.-2\left[7-72 \theta^{\prime \prime 2}+77 \theta^{\prime \prime} 4-\eta^{\prime \prime 2}\left(5-56 \theta^{\prime \prime 2}+63 \theta^{\prime \prime 4}\right)\right] \cos 2 \mathrm{~g}^{\prime \prime}\right\}
\end{aligned}
$$




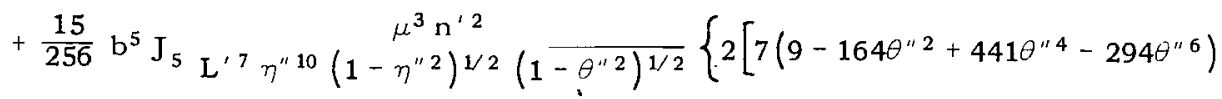

$$
\begin{aligned}
& \left.-\eta^{\prime \prime 2}\left(77-1445 \theta^{\prime \prime 2}+3955 \theta^{\prime \prime} 4-2667 \theta^{\prime \prime 6}\right)+3 \eta^{\prime \prime}\left(6-119 \theta^{\prime \prime} 2+336 \theta^{\prime \prime} 4-231 \theta^{\prime \prime} 6\right)\right] \sin g^{\prime \prime} \\
& \left.-7\left(1-\eta^{\prime \prime 2}\right)\left(1-\theta^{\prime \prime}\right)\left[3-37 \theta^{\prime \prime 2}+42 \theta^{\prime \prime} 4-\eta^{\prime \prime 2}\left(2-27 \theta^{\prime \prime 2}+33 \theta^{\prime \prime 4}\right)\right] \sin 3 g^{\prime \prime}\right\} \text {, }
\end{aligned}
$$

$$
\begin{aligned}
& \frac{\partial \mathrm{F}_{2}^{\prime \prime}}{\partial \mathrm{g}^{\prime}}=-\frac{15}{8 \epsilon} \mathrm{n}^{\prime} L^{\prime}\left(\frac{\mathrm{n}_{\overparen{C}}}{\mathrm{n}^{\prime}}\right)^{2}\left(1-\eta^{\prime \prime 2}\right)\left(1-\theta^{\prime \prime} 2\right) \sin 2 \mathrm{~g}^{\prime \prime} \\
& -\frac{3}{8} \mathrm{~b}^{3} \mathrm{~J}_{3} \mu \mathrm{n}^{\prime 2}\left(1-\eta^{\prime \prime 2}\right)^{1 / 2}\left(1-\theta^{\prime \prime 2}\right)^{1 / 2}\left(1-5 \theta^{\prime \prime 2}\right) \cos \mathrm{g}^{\prime \prime}
\end{aligned}
$$

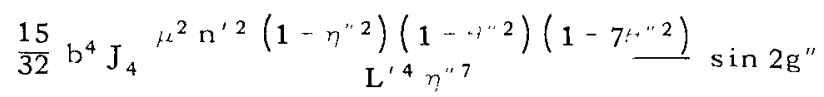

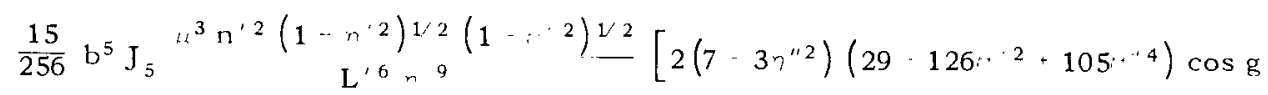

$$
\begin{aligned}
& \left.7\left(1-\eta^{\prime \prime 2}\right)(1 \quad, 2)\left(7-15^{\prime \prime 2}\right) \cos 3 \mathrm{~g}\right]
\end{aligned}
$$

$\frac{\partial S_{1}^{\prime}}{\partial h^{\prime}} \quad \frac{1}{n_{\overparen{C}}^{*}} F_{29}^{\prime} \quad \frac{3 L^{\prime}}{32 \epsilon}\left(\frac{n_{\overparen{C}}^{2}}{n_{\mathbb{C}}^{*} n^{\prime}}\right)\left\{2\left(5-3 n^{2}\right)(1,2) \cos 2 h\right.$

$$
\begin{aligned}
& \left.+5\left(1 n^{2}\right)\left[(1+\cdots)^{2} \cos 2\left(g^{\prime}+h\right)+\left(1-\mu^{\prime \prime}\right)^{2} \cos 2\left(g^{\prime}-h^{\prime \prime}\right)\right]\right\} \\
& \quad-\frac{3}{2} \frac{n^{\prime 2}}{n_{\mathbb{C}}^{*}} b^{2} J_{22} \frac{1-r^{\prime \prime 2}}{\eta^{\prime 3}} \cos 2 h^{\prime \prime},
\end{aligned}
$$

$\frac{\partial S_{1}^{\prime}}{\partial \mathrm{g}^{\prime}}=-\frac{15}{32 \mathfrak{L}}\left(\frac{\mathrm{n}_{\mathbb{C}}^{2}}{\mathrm{n}_{\mathbb{C}}^{*} \Pi^{\prime}}\right)\left(1-\eta^{\prime \prime}\right) \mathrm{L}^{\prime}\left[\left(1+{H^{\prime \prime}}^{2}\right)^{2} \cos 2\left(\mathrm{~g}^{\prime \prime}+\mathrm{h}^{\prime \prime}\right)-\left(1-\xi^{\prime \prime}\right)^{2} \cos 2\left(\mathrm{~g}^{\prime \prime}-\mathrm{h}^{\prime \prime}\right)\right]$,

and

$$
\frac{\partial \mathrm{S}_{1}^{\prime}}{\partial \mathrm{G}^{\prime \prime}}=-\frac{3}{32 \epsilon}\left(\frac{\mathrm{n}_{\mathbb{C}}^{2}}{\mathrm{n}_{\mathbb{C}}^{*} \mathrm{n}^{\prime}}\right) \frac{1}{\eta} \cdot\left[2\left(5 \theta^{\prime \prime} 2-3 \eta^{\prime \prime}\right) \sin 2 \mathrm{~h}^{\prime \prime}-5\left(1+\theta^{\prime \prime}\right)\left(\eta^{\prime \prime 2}+\theta^{\prime \prime}\right) \sin 2\left(\mathrm{~g}^{\prime \prime}+\mathrm{h}^{\prime \prime}\right)\right.
$$




$$
\left.+5\left(1-\theta^{\prime \prime}\right)\left(\eta^{\prime \prime 2}-\theta^{\prime \prime}\right) \sin 2\left(g^{\prime \prime}-h^{\prime \prime}\right)\right]-\frac{3}{4} \frac{n^{\prime 2} b^{2} J_{22}}{n_{\mathbb{C}}^{*} \eta^{\prime \prime} L^{\prime}}\left(-3+5 \theta^{\prime \prime}\right) \sin 2 h^{\prime \prime} .
$$

From these equations and Equations 74 and 75, it can be shown that

$$
\begin{aligned}
& \mathrm{S}_{2}^{\prime} \text { (coupling) }=\frac{9}{4096 \epsilon^{2}}\left(\frac{\mathrm{n}_{\mathbb{C}}}{n^{\prime}}\right)^{2}\left(\frac{\mathrm{n}_{\mathbb{C}}}{\mathrm{n}_{\mathbb{C}}^{*}}\right)^{2} \frac{\mathrm{L}^{\prime}}{\eta^{\prime \prime}}\left\{-32 \theta^{\prime \prime}\left(1-\theta^{\prime \prime}{ }^{2}\right) \eta^{\prime \prime 2}\left(15-17 \eta^{\prime \prime}\right) \sin 2 \mathrm{~h}^{\prime \prime}\right. \\
& -4 r^{\prime \prime}\left(1-t^{\prime \prime 2}\right)\left(5-3 \eta^{\prime \prime 2}\right)^{2} \sin 4 h^{\prime \prime} \\
& +80\left(1+g^{\prime \prime}\right)^{2}\left(2-3 y^{\prime \prime}\right) \eta^{\prime \prime 2}\left(1-\eta^{\prime \prime 2}\right) \sin \left(2 g^{\prime \prime}+2 h^{\prime \prime}\right) \\
& +80\left(1-z^{\prime \prime}\right)^{2}\left(2+3 r^{\prime}\right) \eta^{\prime 2}\left(1-\eta^{\prime \prime 2}\right) \sin \left(2 g^{\prime \prime}-2 h^{\prime \prime}\right) \\
& +10\left(1+\psi^{\prime \prime}\right)^{2}\left(1-\xi^{\prime \prime}\right)\left(1-r^{\prime \prime 2}\right)\left[5\left(1-H^{\prime \prime}\right)-6 \gamma^{\prime \prime 2}\right] \sin \left(2 g^{\prime \prime}+4 h^{\prime \prime}\right) \\
& +10(1-\cdot)^{2}\left(1+r^{\prime}\right)\left(1-n^{2}\right)\left[5(1+y)-6{ }^{2}\right] \sin (2 g-4 h) \\
& +25\left(1+\theta^{\prime \prime}\right)^{3}\left(1-n^{\prime \prime}\right)\left[\left(1-r^{\prime \prime}\right)-2 r^{\prime \prime}{ }^{2}\right] \sin \left(4 \mathrm{~g}+4 \mathrm{~h}^{\prime \prime}\right) \\
& \left.+25\left(1-\cdots^{\prime \prime}\right)^{3}\left(1-\eta^{2}\right)\left[\left(1+*^{\prime}\right)-2 \eta^{\prime 2}\right] \sin \left(4 g^{\prime}-4 h^{\prime}\right)\right\} \\
& +\frac{9}{256 \in} n^{\prime}+\cdot-4\left(\frac{n_{\overparen{C}}}{n_{\overparen{C}}^{*}}\right)^{2} J_{2} b^{2}\left[4 \cdots(1-\cdots 2)\left(5-3 r^{\prime 2}\right) \sin 2 h\right. \\
& +5\left(1+\theta^{\prime \prime}\right)^{2}\left(1+2 r^{\prime \prime}-5 k^{\prime \prime 2}\right)\left(1-\eta^{\prime \prime 2}\right) \sin \left(2 g^{\prime \prime}+2 h^{\prime}\right) \\
& \left.+5\left(1-\theta^{\prime \prime}\right)^{2}\left(1-2 \ddots^{\prime}-5^{\prime \prime 2}\right)\left(1-\eta^{\prime \prime 2}\right) \sin \left(2 g^{\prime}-2 h^{\prime \prime}\right)\right] \\
& -\frac{9}{512 \epsilon} n^{\prime} r^{-4}\left(\frac{n_{C}}{n_{C}^{*}}\right)^{2} J_{22} b^{2}\left\{-8 \cdot\left(1-\omega^{\prime \prime 2}\right)\left(5-3 r^{2}\right) ! 2 \sin 2 h^{\prime \prime}-\sin 4 h\right. \\
& -20\left(1+4^{\prime \prime}\right)^{2}\left(1-f^{\prime \prime}\right)\left(3-5{ }^{\prime \prime}\right)\left(1-n^{\prime 2}\right) \sin \left(2 \mathrm{~g}^{\prime \prime}+2 \mathrm{~h}^{\prime \prime}\right) \\
& -20\left(1-\theta^{\prime \prime}\right)^{2}\left(1+\theta^{\prime}\right)\left(3+5 t^{\prime \prime}\right)\left(1-\eta^{\prime 2}\right) \sin \left(2 g^{\prime \prime}-2 h^{\prime \prime}\right)
\end{aligned}
$$




$$
\begin{aligned}
& +5\left(1+\theta^{\prime \prime}\right)^{2}\left(1-\theta^{\prime \prime}\right)\left(1+5 \theta^{\prime \prime}\right)\left(1-\eta^{\prime \prime}\right) \sin \left(2 g^{\prime \prime}+4 h^{\prime \prime}\right) \\
& \left.+5\left(1-\theta^{\prime \prime}\right)^{2}\left(1+\theta^{\prime \prime}\right)\left(1-5 \theta^{\prime \prime}\right)\left(1-\eta^{\prime \prime}\right) \sin \left(2 \mathrm{~g}^{\prime \prime}-4 \mathrm{~h}^{\prime \prime}\right)\right\} \\
& +\frac{9}{8}\left(\frac{n^{\prime}}{n_{\overparen{C}}^{*}}\right)^{2} n^{\prime 2} \theta^{\prime \prime} \frac{\left(1-\theta^{\prime \prime 2}\right)}{L^{\prime} \eta^{\prime \prime 7}} J_{2} J_{22} b^{4} \sin 2 h^{\prime \prime}-\frac{9}{16}\left(\frac{n^{\prime}}{n_{\overparen{C}}^{*}}\right)^{2} n^{\prime 2} \frac{\theta^{\prime \prime}\left(1-\theta^{\prime \prime 2}\right)}{L^{\prime} \eta^{\prime \prime}} J_{22}^{2} b^{4} \sin 4 h^{\prime \prime}
\end{aligned}
$$

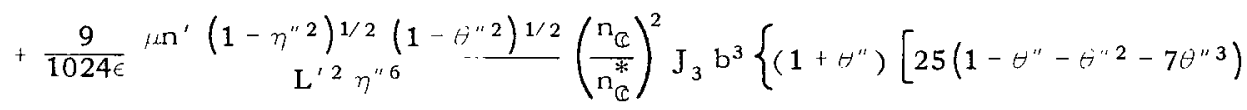

$$
\begin{aligned}
& \left.-\eta^{\prime \prime 2}\left(9+15 H^{\prime \prime}+15 \dagger^{\prime \prime 2}-175^{\prime \prime \prime}\right)\right] \cos \left(\mathrm{g}^{\prime \prime}+2 \mathrm{~h}^{\prime \prime}\right) \\
& +\left(1-n^{\prime}\right)\left[25\left(1+r^{\prime \prime}-4^{\prime \prime 2}+7 c^{\prime \prime 3}\right)\right. \\
& \left.-\eta^{2}\left(9-15 c^{\prime}+15^{\prime \prime 2}+175^{\prime \prime 3}\right)\right] \cos \left(g^{\prime \prime}-2 h^{\prime}\right) \\
& -25\left(1-\eta^{\prime 2}\right)(1+\cdots)^{2}\left(1+2 r^{\prime}-7 r^{\prime 2}\right) \cos \left(3 g+2 h^{\prime \prime}\right) \\
& \left.-25\left(1-\eta^{\prime \prime 2}\right)\left(1-\theta^{\prime \prime}\right)^{2}\left(1-2 \theta^{\prime \prime}-7 t^{\prime \prime 2}\right) \cos \left(3 g^{\prime \prime}-2 h^{\prime \prime}\right)\right\} \\
& +\frac{9}{128}\left(\frac{\mathrm{n}^{\prime}}{\mathrm{n}_{\mathbb{C}}^{*}}\right)^{2} 4 \mathrm{n}^{\prime 2}\left(1-\eta^{\prime \prime 2}\right)^{1 / 2}\left(1-\xi^{\prime \prime 2}\right)^{1 / 2} \mathrm{~L}_{3}^{\prime 3} \mathrm{~J}_{2 \prime} \mathrm{J}_{22} \mathrm{~b}^{5}\left[\left(1+t^{\prime}\right)\left(3-25 t^{\prime \prime}+5 t^{\prime \prime 2}+25 t^{\prime 3}\right) \cos \left(\mathrm{g}^{\prime \prime}+2 \mathrm{~h}^{\prime \prime}\right)\right. \\
& \left.+\left(1+\omega^{\prime}\right)(3+25+5+2-25+3) \cos \left(g-2 h^{\prime \prime}\right)\right]
\end{aligned}
$$

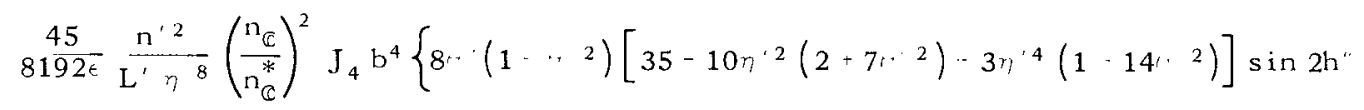$$
+\left(1-\eta^{\prime \prime 2}\right)\left(1+H^{\prime \prime}\right)^{2}\left[35\left(3+4 r^{\prime}-30 t^{\prime \prime 2}-20 r^{\prime \prime 3}+51 y^{\prime \prime} 4\right)\right.
$$$$
\left.-3 \eta^{\prime \prime 2}\left(11+36 \epsilon^{\prime \prime \prime}-126 \theta^{\prime \prime 2}-196 \theta^{\prime \prime} 3+315 \theta^{\prime \prime 4}\right)\right] \sin \left(2 g^{\prime \prime}+2 h^{\prime \prime}\right)
$$$$
+\left(1-\eta^{\prime \prime}\right)\left(1-\theta^{\prime \prime}\right)^{2}\left[35\left(3-4 \theta^{\prime \prime}-30 \theta^{\prime \prime 2}+20 \theta^{\prime \prime} 3+51 \theta^{\prime \prime}\right)\right.
$$$$
\left.-3 \eta^{\prime \prime 2}\left(11-36 \theta^{\prime \prime}-126 \theta^{\prime \prime 2}+196 \theta^{\prime \prime} 3+315 \theta^{\prime \prime}\right)\right] \sin \left(2 \mathrm{~g}^{\prime \prime}-2 \mathrm{~h}^{\prime \prime}\right)
$$ 


$$
\begin{aligned}
& -35\left(1-\eta^{\prime \prime 2}\right)^{2}\left(1-\theta^{\prime \prime}\right)\left[\left(1+\theta^{\prime \prime}\right)^{2}\left(1+2 \theta^{\prime \prime}-9 \theta^{\prime \prime}\right) \sin \left(4 \mathrm{~g}^{\prime \prime}+2 \mathrm{~h}^{\prime \prime}\right)\right. \\
& \left.\left.+\left(1-\theta^{\prime \prime}\right)^{2}\left(1-2 \theta^{\prime \prime}-9 \theta^{\prime \prime}\right) \sin \left(4 g^{\prime \prime}-2 h^{\prime \prime}\right)\right]\right\} \\
& +\frac{45}{512}\left(\frac{n^{\prime}}{n_{\overparen{C}}^{*}}\right)^{2} \frac{n^{\prime 3}\left(1-\theta^{\prime \prime 2}\right)}{L^{\prime 2} \eta^{\prime \prime 11}} J_{4} J_{22} b^{6}\left[4\left(5-3 \eta^{\prime \prime 2}\right) \theta^{\prime \prime}\left(3-7 \theta^{\prime \prime 2}\right) \sin 2 h^{\prime \prime}\right. \\
& +\left(1-\eta^{\prime \prime}\right)\left(1+\theta^{\prime \prime}\right)\left(3-19 \theta^{\prime \prime}-7 \theta^{\prime \prime} 2+35 \theta^{\prime \prime}\right) \sin \left(2 g^{\prime \prime}+2 h^{\prime \prime}\right) \\
& \left.+\left(1-\eta^{\prime \prime 2}\right)\left(1-t^{\prime \prime}\right)\left(3+19 \theta^{\prime \prime}-7 \theta^{\prime \prime} 2-35 H^{\prime \prime}\right) \sin \left(2 g^{\prime \prime}-2 h^{\prime \prime}\right)\right]
\end{aligned}
$$

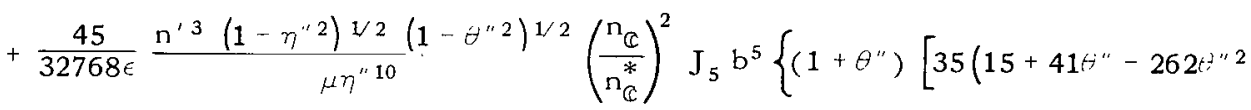

$$
\begin{aligned}
& \left.-346 \theta^{\prime \prime 3}+519 \theta^{\prime \prime 4}+321 \epsilon^{\prime \prime} 5\right)+14 \eta^{\prime \prime 2}\left(259-255 t^{\prime \prime}-566 \theta^{\prime \prime 2}+1710 \theta^{\prime \prime 3}-525 t^{\prime \prime 4}-1455 \theta^{\prime \prime}\right) \\
& \left.-\eta^{\prime \prime}\left(1559-1015 r^{\prime \prime}-5446 \theta^{\prime \prime 2}+7350 \theta^{\prime \prime} 3+735 t^{\prime \prime 4}-5775 \nvdash^{\prime \prime}\right)\right] \cos \left(\mathrm{g}^{\prime \prime}+2 \mathrm{~h}^{\prime \prime}\right) \\
& +\left(1-\theta^{\prime \prime}\right)\left[35\left(15-41 \theta^{\prime \prime}-262 \theta^{\prime \prime} 2+346 \theta^{\prime \prime} 3+519 \theta^{\prime \prime} 4-321 \theta^{\prime \prime}\right)\right. \\
& +14 \eta^{\prime \prime 2}\left(259+255 \theta^{\prime \prime}-566 \theta^{\prime \prime 2}-1710 \theta^{\prime \prime} 3-525 \theta^{\prime \prime} 4+1455 \theta^{\prime \prime}\right) \\
& \left.-\eta^{\prime \prime}\left(1559+1015 \theta^{\prime \prime}-5446 \theta^{\prime \prime 2}-7350 \theta^{\prime \prime} 3+735 \theta^{\prime \prime} 4+5775 \theta^{\prime \prime}\right)\right] \cos \left(\mathrm{g}^{\prime \prime}-2 \mathrm{~h}^{\prime \prime}\right) \\
& -14\left(1+\theta^{\prime \prime 2}\right)\left[5\left(9-7 \theta^{\prime \prime}-119 \theta^{\prime \prime 2}+15 \theta^{\prime \prime 3}+174 t^{\prime \prime 4}\right)-\eta^{\prime \prime 2}\left(179-14 \theta^{\prime \prime}-1320 \theta^{\prime \prime} 2+30 \theta^{\prime \prime} 3+1605^{\prime \prime} 4\right)\right. \\
& \left.+3 \eta^{\prime \prime}\left(18+7 \theta^{\prime \prime}-135 \theta^{\prime \prime 2}-15 \theta^{\prime \prime}+165 \theta^{\prime \prime}\right)\right] \cos \left(3 g^{\prime}+2 h^{\prime \prime}\right) \\
& -14\left(1-\theta^{\prime \prime}\right)^{2}\left[5\left(9+7 \theta^{\prime \prime}-119 \theta^{\prime \prime}-15 \theta^{\prime \prime} 3+174 \theta^{\prime \prime}\right)\right. \\
& -\eta^{\prime \prime 2}\left(179+14 \theta^{\prime \prime}-1320 \theta^{\prime \prime 2}-30 \theta^{\prime \prime} 3+1605 \theta^{\prime \prime}\right) \\
& \left.+3 \eta^{\prime \prime} 4\left(18-7 \theta^{\prime \prime}-135 \theta^{\prime \prime 2}+15 \theta^{\prime \prime} 3+165 \theta^{\prime 4}\right)\right] \cos \left(3 g^{\prime \prime}-2 h^{\prime \prime}\right) \\
& +105\left(1-\eta^{\prime \prime 2}\right)\left(1-\theta^{\prime \prime}\right)\left[1-9 \theta^{\prime \prime} 2-\eta^{\prime \prime 2}\left(3-11 \theta^{\prime \prime 2}\right)\right] \\
& \left.\times\left[\left(1+\theta^{\prime \prime}\right)^{2} \cos \left(5 g^{\prime \prime}+2 h^{\prime \prime}\right)+\left(1-\theta^{\prime \prime}\right)^{2} \cos \left(5 g^{\prime \prime}-2 h^{\prime \prime}\right)\right]\right\}
\end{aligned}
$$




$$
\begin{aligned}
& +\frac{45}{4096}\left(\frac{n^{\prime}}{n_{\overparen{C}}^{*}}\right)^{2} n^{\prime 4}\left(1-\eta^{\prime \prime 2}\right)^{1 / 2}\left(1-\theta^{\prime \prime 2}\right)^{1 / 2} \mathrm{~J}_{5} \mathrm{~J}_{22} \mathrm{~b}^{7}\left\{2 ( 7 - 3 \eta ^ { \prime \prime 2 } ) ( 2 9 - 1 2 6 \theta ^ { \prime \prime 2 } + 1 0 5 ^ { \prime 4 } ) \left[\left(1+\theta^{\prime \prime}\right)\left(3-5 \theta^{\prime \prime}\right) \cos \left(\mathrm{g}^{\prime \prime}+2 \mathrm{~h}^{\prime \prime}\right)\right.\right. \\
& \left.+\left(1-\theta^{\prime \prime}\right)\left(3+5 \dot{\circ}^{\prime \prime}\right) \cos \left(\mathrm{g}^{\prime \prime}-2 \mathrm{~h}^{\prime \prime}\right)\right]-7\left(1-\eta^{\prime \prime}\right)\left(1-\theta^{\prime \prime}\right)\left(7-15 \theta^{\prime \prime}\right)\left[\left(1+\theta^{\prime \prime}\right)\left(3-5 \theta^{\prime \prime}\right) \cos \left(3 \mathrm{~g}^{\prime \prime}+2 \mathrm{~h}^{\prime \prime}\right)\right. \\
& \left.\left.+\left(1-\theta^{\prime \prime}\right)\left(3+5 \theta^{\prime \prime}\right) \cos \left(3 g^{\prime \prime}-2 h^{\prime \prime}\right)\right]\right\} \\
& \mathrm{F}_{3}^{\prime \prime}(\operatorname{coup} 1 \text { ing })=\frac{9}{128}\left(\frac{\mathrm{n}_{\overparen{C}}}{\mathrm{n}^{\prime}}\right)^{3}\left(\frac{\mathrm{n}_{\mathbb{C}}}{\mathrm{n}_{\mathbb{C}}^{*}}\right) \frac{\mathrm{n}^{\prime 2}}{\epsilon^{2}} \mathrm{a}^{\prime 2} \sqrt{1-\mathrm{e}^{\prime \prime}} \cos \mathrm{I}^{\prime \prime}\left[\left(2+33 \mathrm{e}^{\prime \prime}\right)\right. \\
& \left.-\left(2-17 e^{\prime \prime}\right) \cos ^{2} \cdot I^{\prime \prime}+15 e^{\prime \prime} \sin ^{2} I^{\prime \prime} \cos 2 g^{\prime \prime}\right] \\
& +\frac{9}{32}\left(\frac{n_{\mathbb{C}}}{n^{\prime}}\right)\left(\frac{n_{\mathbb{C}}}{n_{\overparen{C}}^{*}}\right) n_{\in\left(1-e^{\prime \prime}\right)^{2} \sin ^{2} I^{\prime \prime} \cos I^{\prime \prime}} J_{22} b^{2}\left[2\left(2+3 e^{\prime \prime 2}\right)+15 e^{\prime 2} \cos 2 g^{\prime \prime}\right] \\
& +\frac{9}{4}\left(\frac{n^{\prime}}{n_{\mathbb{C}}^{*}}\right)_{\mathrm{a}^{\prime 2}\left(1-\mathrm{e}^{\prime 2}\right)^{7 / 2} \sin ^{2} I \cos I} J_{22}^{2} b^{4},
\end{aligned}
$$

and, of course,

$$
\mathrm{S}_{3}{ }^{\prime}(\text { coupling })=0
$$

It is important to note that $S_{2}^{\prime}$ (coupling) is made up of second-order terms, although it is obtained by multiplying a second-order quantity by a first-order quantity.

The next few sections are devoted to the computation of $\mathrm{F}_{3}{ }^{\prime}$. From that computation various additional parts of $\mathrm{F}_{3}{ }^{\prime \prime}, \mathrm{S}_{3}{ }^{\prime}$, and $\mathrm{S}_{2}^{\prime}$ are then derived.

\section{THE RADIATION PRESSURE}

For this computation, the shadow effect will be neglected. Its inclusion would require, of course, the introduction of a shadow function due both to the moon and to the earth.

Consider $v$ to be the absolute value of the acceleration of the orbiter arising from the solar radiation. Then, the disturbing function for the radiation pressure will be given by (see the earlier section, "The Angles $\mathrm{S}_{10}^{\prime}$ and $\mathrm{s}_{13}$ ")

$$
\mathbf{R}_{o}=-\sigma \mathrm{r} \cos \mathrm{S}_{13}^{\prime \prime}
$$

or, neglecting the inclination of the lunar equator to the sun's orbit,

$$
R_{\sigma}=-\sigma r\left[\cos (f+g) \cos \left(\Omega-\lambda_{\odot}\right)-\sin (f+g) \sin \left(\Omega-\lambda_{\odot}\right) \cos I\right] .
$$


The elimination of short-period terms gives

$$
\mathbf{R}_{\sigma}^{\prime}=\frac{3}{2} \sigma \mathrm{a}^{\prime} \mathrm{e}^{\prime}\left[\cos ^{2} \frac{\mathrm{I}^{\prime}}{2} \cos \left(\mathrm{g}^{\prime}+\mathrm{h}^{\prime}+\lambda_{\oplus}-\lambda_{\odot}\right)+\sin ^{2} \frac{\mathrm{I}^{\prime}}{2} \cos \left(\mathrm{g}^{\prime}-\mathrm{h}^{\prime}+\lambda_{\odot}-\lambda_{\oplus}\right)\right],
$$

where the node $\Omega$ was written in terms of the canonical variable $h$. There is no secular contribution from this effect, that is, $F_{3 s}^{\prime}$ (radiation) $=R_{\sigma s}^{\prime}=0$. On the other hand, since there are no terms strictly independent of time, the contribution to $\mathrm{S}_{2}^{\prime}$ is zero, or

$$
S_{2}^{\prime}(\text { radiation })=0 .
$$

Therefore,

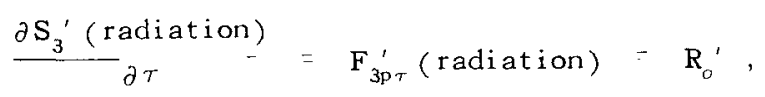

or

$$
\mathrm{S}_{3}^{\prime} \text { (radiation) }=\frac{3}{2} \frac{\sigma \mathrm{a}^{\prime \prime} \mathrm{e}^{\prime \prime}}{\mathrm{n}_{\mathbb{C}}^{*}-\mathrm{n}_{\oplus}^{*}}\left[\cos ^{2} \frac{\mathrm{I}}{2} \sin \left(\mathrm{g}+\mathrm{h}^{\prime}+\lambda_{\Psi}-\lambda_{\odot}\right)-\sin ^{2} \frac{\mathrm{I}^{\prime \prime}}{2} \sin \left(\mathrm{g}^{\prime \prime}-\mathrm{h}^{\prime}+\lambda_{\mathrm{e}}-\lambda_{\oplus}\right)\right] \text {, }
$$

where

$$
\begin{aligned}
& \lambda_{\oplus}=n_{\oplus}^{*}+\text { const. }, \\
& \lambda_{\oplus}-n_{C}^{*}+\text { const. },
\end{aligned}
$$

and $n_{\oplus}^{*}$ and $n_{\overparen{C}}^{*}$ are, respectively, the mean motion in longitude of the sun around the moon and of the moon around the earth. Of course, $n_{\mathbb{C}}^{*}-n_{\oplus}^{*} \simeq n_{\mathbb{C}}^{*}$ is a good approximation.

\section{THE SECOND LEGENDRE POLYNOMIAL FOR THE}

SUN'S GRAVITATIONAL PERTURBATIONS

The most important term in the disturbing function due to the sun's gravity is

$$
\frac{\mu_{3}}{r_{3}}\left(\frac{r}{r_{3}}\right)^{2} P_{2}\left(\cos S_{13}^{\prime \prime}\right), \quad \text { (see Equation 20) }
$$


where, for the computation of $\mathrm{S}_{13}$, the sun can be considered to move along the moon's equator. Thus,

$$
\cos S_{13}^{\prime \prime}=\cos (f+g) \cos \left(\Omega-\lambda_{\odot}\right)-\sin (f+g) \sin \left(\Omega-\lambda_{\odot}\right) \cos I .
$$

On the other hand, neglecting the mass of the moon,

$$
n_{\oplus}^{2} a_{3}^{3}=k^{2}\left(m_{\odot}+m_{\oplus}\right)=k^{2} m_{\odot}\left(1+\frac{m_{\oplus}}{m_{\odot}}\right)
$$

and since the ratio $m_{\oplus} / m_{\odot}$ can be neglected,

$$
\mathrm{n}_{\oplus}^{2} \mathrm{a}_{3}^{3}=\mu_{3} .
$$

Furthermore, if the eccentricity of the earth orbit is neglected,

$$
\begin{aligned}
F_{3}(\operatorname{sun})= & n_{\oplus}^{2} r^{2} P_{2}\left(\cos S_{13}^{\prime \prime}\right) \\
& =\frac{1}{2} n_{\oplus}^{2} r^{2}\left(3 \cos ^{2} S_{13}^{\prime \prime}-1\right) \\
= & \frac{3}{2} n_{\oplus}^{2} r^{2}\left[\cos ^{2}(f+g) \cos ^{2}\left(\Omega-\lambda_{\odot}\right)-2 \sin (f+g) \cos (f+g) \sin \left(\Omega-\lambda_{\odot}\right) \cos \left(\Omega-\lambda_{\odot}\right) \cos I\right. \\
& \left.\quad+\sin ^{2}(f+g) \sin ^{2}\left(\Omega-\lambda_{\odot}\right) \cos ^{2} I\right]-\frac{1}{2} n_{\oplus}^{2} r^{2}
\end{aligned}
$$

Rewritten, Equation 93 becomes

$$
\begin{aligned}
F_{3}(\operatorname{sun}) & -\frac{3}{2} n_{\oplus}^{2} r^{2}\left[\left(\cos ^{2} f \cos ^{2} g+\sin ^{2} f \sin ^{2} g\right) \cos ^{2}\left(\Omega-\lambda_{\odot}\right)\right. \\
& -2\left(-\sin ^{2} f \sin g \cos g+\cos ^{2} f \sin g \cos g\right) \sin \left(\Omega-\lambda_{\odot}\right) \cos \left(\Omega-\lambda_{\odot}\right) \cos I \\
& \left.+\left(\sin ^{2} f \cos ^{2} g+\cos ^{2} f \sin ^{2} g\right) \sin ^{2}\left(\Omega-\lambda_{\odot}\right) \cos ^{2} I\right]-\frac{1}{2} n_{\oplus}^{2} r^{2}+\frac{3}{2} n_{\oplus}^{2} r^{2} \sin f \cos f \cdot Q,
\end{aligned}
$$

where $Q$ is independent of $f$. It follows that

$$
\begin{aligned}
\mathrm{F}_{3}^{\prime} \text { (sun) }= & \frac{1}{32} \mathrm{n}_{\oplus}^{2} \mathrm{a}^{\prime \prime 2}\left[-2\left(2+3 \mathrm{e}^{\prime \prime}\right)\left(1-3 \cos ^{2} \mathrm{I}^{\prime \prime}\right)+30 \mathrm{e}^{\prime \prime} \sin ^{2} \mathrm{I}^{\prime \prime} \cos 2 \mathrm{~g}^{\prime \prime}\right. \\
+ & 6\left(2+3 \mathrm{e}^{\prime \prime 2}\right) \sin ^{2} \mathrm{I}^{\prime \prime} \cos 2\left(\mathrm{~h}^{\prime \prime}+\lambda_{\oplus}-\lambda_{\odot}\right)+15 \mathrm{e}^{\prime \prime 2}\left(1+\cos \mathrm{I}^{\prime \prime}\right)^{2} \cos 2\left(\mathrm{~g}^{\prime \prime}+\mathrm{h}^{\prime \prime}+\lambda_{\oplus}-\lambda_{\odot}\right) \\
& \left.+15 \mathrm{e}^{\prime 2}\left(1-\cos \mathrm{I}^{\prime \prime}\right)^{2} \cos 2\left(\mathrm{~g}^{\prime \prime}-\mathrm{h}^{\prime \prime}-\lambda_{\oplus}+\lambda_{\odot}\right)\right] .
\end{aligned}
$$


The secular contribution is

$$
\mathrm{F}_{3}^{\prime \prime}(\operatorname{sun})=\frac{1}{16} \mathrm{n}_{\oplus}^{2} \mathrm{a}^{\prime 2}\left[-\left(2+3 \mathrm{e}^{\prime \prime}\right)\left(1-3 \cos ^{2} \mathrm{I}^{\prime \prime}\right)+15 \mathrm{e}^{\prime 2}\left(1-\cos ^{2} \mathrm{I}^{\prime \prime}\right) \cos 2 \mathrm{~g}^{\prime \prime}\right] \text {. }
$$

There is no contribution to $S_{2}{ }^{\prime}$, and the contribution to $S_{3}^{\prime}$ is

$$
\begin{aligned}
S_{3}{ }^{\prime}(\operatorname{sun})= & \frac{3}{64} \frac{\mathrm{n}_{\oplus}{ }^{2} \mathbf{a}^{\prime \prime}}{\mathrm{n}_{\overparen{C}}^{*}-\mathrm{n}_{\oplus}^{*}}\left[2\left(2+3 \mathrm{e}^{\prime \prime}\right) \sin ^{2} \mathrm{I}^{\prime \prime} \sin 2\left(\mathrm{~h}^{\prime \prime}+\lambda_{\oplus}-\lambda_{\odot}\right)\right. \\
& \left.+5 \mathrm{e}^{\prime \prime 2}\left(1+\cos \mathrm{I}^{\prime \prime}\right)^{2} \sin 2\left(\mathrm{~h}^{\prime \prime}+\mathrm{g}^{\prime \prime}+\lambda_{\oplus}-\lambda_{\odot}\right)+5 \mathrm{e}^{\prime \prime 2}\left(1-\cos \mathrm{I}^{\prime \prime}\right)^{2} \sin 2\left(\mathrm{~h}^{\prime \prime}-\mathrm{g}^{\prime \prime}+\lambda_{\oplus}-\lambda_{\odot}\right)\right] .
\end{aligned}
$$

\section{THE THIRD LEGENDRE POLYNOMIAL FOR THE}

\section{EARTH'S GRAVITATIONAL PERTURBATIONS}

From Equation 20,

$$
F_{3}(\text { earth })=\frac{\mu_{2}}{r_{0}}\left(\frac{r}{r_{0}}\right)^{3} P_{3}\left(\cos S_{10}^{\prime}\right)
$$

Now,

$$
n_{\mathbb{C}}^{2} a_{\mathbb{C}}^{3}=k^{2}\left(m_{\oplus}+m_{\mathbb{C}}\right)=k^{2} m_{\oplus}\left(1+\frac{m_{\mathscr{C}}}{m_{\oplus}}\right)=\mu_{2} \epsilon,
$$

so that, considering the moon's orbit to be circular,

$F_{3}($ earth $)=\frac{n_{\mathbb{C}}^{2} a_{\mathbb{C}}^{3}}{\epsilon} \frac{r^{3}}{a_{\mathbb{C}}^{4}} P_{3}\left(\cos S_{10}^{\prime}\right)=\frac{n_{\mathbb{C}}^{2}}{\epsilon a_{\mathbb{C}}} r^{3} P_{3}\left(\cos S_{10}^{\prime}\right)=\frac{1}{2} \frac{n_{\mathbb{C}}^{2}}{\epsilon a_{\mathbb{C}}} r^{3}\left(5 \cos ^{3} S_{10}^{\prime}-3 \cos S_{10}^{\prime}\right)$.

If

$$
A_{A}=\frac{1}{2}[(1+\cos I) \cos (g+h)+(1-\cos I) \cos (g-h)]
$$

and

$$
B_{\oplus}=\frac{1}{2}[(1+\cos I) \sin (g+h)+(1-\cos I) \sin (g-h)],
$$

then

$$
\cos S_{10}^{\prime}=A_{\oplus} \cos f-B_{\oplus} \sin f
$$


so that

$F_{3}($ earth $)=\frac{1}{2} \frac{n_{\overparen{C}}^{2}}{\epsilon a_{\mathbb{C}}} r^{3}\left[5\left(A_{\oplus}^{3} \cos ^{3} f-3 A_{\oplus}^{2} B_{\oplus} \cos ^{2} f \sin f\right.\right.$

$$
\left.\left.+3 A_{\oplus} B_{\oplus}^{2} \cos f \sin ^{2} f-B_{\oplus}^{3} \sin ^{3} f\right)-3\left(A_{\oplus} \cos f-B_{\oplus} \sin f\right)\right] \cdot(100)
$$

It follows that

$$
F_{3}^{\prime}(\text { earth })=-\frac{5}{16} \frac{n_{\mathbb{C}}^{2} a^{\prime 3} e^{\prime}}{\epsilon a_{\mathbb{C}}}\left\{5\left[\left(3+4 e^{\prime 2}\right) A_{\oplus}^{\prime 3}+3 A_{\oplus}^{\prime} B_{\oplus}^{\prime 2}\left(1-e^{\prime 2}\right)\right]-3 A_{\oplus}^{\prime}\left(4+3 e^{\prime 2}\right)\right\},
$$

where

$$
\begin{aligned}
& A_{\oplus}^{\prime 3}=\frac{1}{32}\left[3\left(1-\theta^{\prime 2}\right)\left(1-\theta^{\prime}\right) \cos \left(g^{\prime}-3 h^{\prime}\right)+\left(1-\theta^{\prime}\right)^{3} \cos \left(3 g^{\prime}-3 h^{\prime}\right)\right. \\
& +3\left(1-\prime^{\prime 2}\right)\left(1-\theta^{\prime}\right) \cos \left(3 g^{\prime}-h^{\prime}\right)+3\left(1-\theta^{\prime}\right)\left(3+2 \theta^{\prime}+3 t^{\prime 2}\right) \cos \left(g^{\prime}-h^{\prime}\right) \\
& +3\left(1+f^{\prime}\right)\left(3-2^{\prime \prime}+3 l^{\prime 2}\right) \cos \left(g^{\prime}+h^{\prime}\right)+3\left(1-g^{\prime} 2\right)\left(1+g^{\prime}\right) \cos \left(3 g^{\prime}+h^{\prime}\right) \\
& \left.+3\left(1-\theta^{\prime 2}\right)\left(1+\theta^{\prime}\right) \cos \left(g^{\prime}+3 h^{\prime}\right)+\left(1+\theta^{\prime}\right)^{3} \cos \left(3 g^{\prime}+3 h^{\prime}\right)\right], \\
& \mathrm{A}_{\oplus}^{\prime}-\frac{1}{2}\left(1+\theta^{\prime}\right) \cos \left(\mathrm{g}^{\prime}+\mathrm{h}^{\prime}\right)+\frac{1}{2}\left(1-0^{\prime}\right) \cos \left(\mathrm{g}^{\prime}-\mathrm{h}^{\prime}\right)
\end{aligned}
$$

and

$$
\begin{aligned}
& \mathrm{A}_{q^{\prime}} \mathrm{B}_{q^{\prime}}{ }^{2}=\frac{1}{32}\left[\left(1+y^{\prime}\right)\left(3-2 f^{\prime \prime}+3 t^{\prime 2}\right) \cos \left(\mathrm{g}^{\prime}+\mathrm{h}^{\prime}\right)\right. \\
& +\left(1-\theta^{\prime}\right)\left(3+2 \theta^{\prime}+3 t^{\prime 2}\right) \cos \left(g^{\prime}-h^{\prime}\right)-3\left(1-0^{\prime}\right)\left(1-\theta^{\prime 2}\right) \cos \left(3 g^{\prime}-h^{\prime}\right) \\
& +\left(1-\theta^{\prime}\right)\left(1-\theta^{\prime 2}\right) \cos \left(g^{\prime}-3 h^{\prime}\right)-\left(1-\theta^{\prime}\right)^{3} \cos \left(3 g^{\prime}-3 h^{\prime}\right) \\
& -\left(1+\theta^{\prime}\right)^{3} \cos \left(3 g^{\prime}+3 h^{\prime}\right)-3\left(1+\theta^{\prime}\right)\left(1-\theta^{\prime 2}\right) \cos \left(3 g^{\prime}+h^{\prime}\right) \\
& \left.+\left(1+\theta^{\prime}\right)\left(1-\theta^{\prime 2}\right) \cos \left(g^{\prime}+3 h^{\prime}\right)\right] .
\end{aligned}
$$

The contributions to $\mathrm{F}_{3}{ }^{\prime \prime}$ and to $\mathrm{S}_{3}^{\prime}$ are both zero. The contribution to $\mathrm{S}_{2}{ }^{\prime}$ is then given by

$$
S_{2}^{\prime}(\text { earth })=-\frac{1}{n_{C}^{*}} \int F_{3}^{\prime}(\text { earth }) d^{\prime},
$$


or

$$
\begin{aligned}
& S_{2}^{\prime}(\text { earth })=\frac{5}{1536}\left(\frac{n_{\mathbb{C}}}{n_{\mathbb{C}}^{*}}\right) \frac{n_{\mathbb{C}} a^{\prime \prime 3} e^{\prime \prime}}{a_{\mathbb{C}} \epsilon}\left[-9\left(1+\cos I^{\prime \prime}\right)\left(1+10 \cos I^{\prime \prime}-15 \cos ^{2} I^{\prime \prime}\right)\left(4+3 e^{\prime \prime 2}\right) \sin \left(g^{\prime \prime}+h^{\prime \prime}\right)\right. \\
& +9\left(1-\cos I^{\prime \prime}\right)\left(1-10 \cos I^{\prime \prime}-15 \cos ^{2} I^{\prime \prime}\right)\left(4+3 e^{\prime 2}\right) \sin \left(g^{\prime \prime}-h^{\prime \prime}\right) \\
& +15 \sin ^{2} I^{\prime \prime}\left(1+\cos I^{\prime \prime}\right)\left(4+3 e^{\prime \prime}\right) \sin \left(g^{\prime \prime}+3 h^{\prime \prime}\right)-15 \sin ^{2} I^{\prime \prime}\left(1-\cos I^{\prime \prime}\right)\left(4+3 e^{\prime 2}\right) \sin \left(g^{\prime \prime}-3 h^{\prime \prime}\right) \\
& +315 \sin ^{2} I^{\prime \prime}\left(1+\cos I^{\prime \prime}\right) e^{\prime 2} \sin \left(3 g^{\prime \prime}+h^{\prime \prime}\right)-315 \sin ^{2} I^{\prime \prime}\left(1-\cos I^{\prime \prime}\right) e^{\prime \prime 2} \sin \left(3 g^{\prime \prime}-h^{\prime \prime}\right) \\
& \left.+35\left(1+\cos I^{\prime \prime}\right)^{3} e^{\prime \prime 2} \sin \left(3 g^{\prime \prime}+3 h^{\prime \prime}\right)-35\left(1-\cos I^{\prime \prime}\right)^{3} e^{\prime \prime 2} \sin \left(3 g^{\prime \prime}-3 h^{\prime \prime}\right)\right]
\end{aligned}
$$

\section{THE ECCENTRICITY OF THE MOON'S ORBIT}

The correction for the eccentricity of the moon's orbit is given by

$$
F_{3}\left(e_{\mathbb{C}}\right)=\frac{n_{\mathbb{C}}^{2}}{\epsilon}\left[\left(\frac{a_{\mathbb{C}}}{r_{\mathbb{C}}}\right)^{3}-1\right] r^{2} P_{2}\left(\cos \widetilde{S}_{10}\right)
$$

or, keeping only the first power of $e_{\mathbb{C}}$,

$$
\begin{aligned}
F_{3}\left(e_{\mathscr{C}}\right) & =\frac{3}{2} e_{\mathbb{C}} \frac{n_{\mathscr{C}}^{2}}{\epsilon} r^{2} \cos l_{\oplus} P_{2}\left(\cos \widetilde{S}_{10}\right) \\
& =\frac{3}{4} \frac{e_{\mathbb{C}} n_{\overparen{C}}^{2}}{\epsilon} r^{2}\left(3 \cos ^{2} \tilde{S}_{10}-1\right) \cos l_{\oplus},
\end{aligned}
$$

where the mean anomaly $l_{\oplus}$ is $l_{\oplus}=\mathrm{n}_{\mathbb{C}} \mathrm{t}+$ const. Then,

$F_{3}^{\prime}\left(e_{\mathbb{C}}\right)=\frac{3}{64} \frac{n_{\mathbb{C}}^{2} a^{\prime 2}}{\epsilon} e_{\mathbb{C}}\left[-2\left(2+3 e^{\prime 2}\right)\left(1-3 \cos ^{2} I^{\prime}\right)+30 e^{\prime 2} \sin ^{2} I^{\prime} \cos 2 g^{\prime}+6\left(2+3 e^{\prime 2}\right) \sin ^{2} I^{\prime} \cos 2 h^{\prime}\right.$

$$
\left.+15 e^{\prime 2}\left(1+\cos I^{\prime}\right)^{2} \cos \left(2 g^{\prime}+2 h^{\prime}\right)+15 e^{\prime 2}\left(1-\cos I^{\prime}\right)^{2} \cos \left(2 g^{\prime}-2 h^{\prime}\right)\right] \cos l_{\oplus} \cdot(10
$$

There are no contributions to $S_{2}^{\prime}$ or $F_{3}^{\prime \prime}$. The contribution to $S_{3}^{\prime}$ is

$$
\begin{aligned}
\mathrm{S}_{3}^{\prime}\left(\mathrm{e}_{\mathbb{C}}\right)=\frac{3}{64} \frac{\mathrm{n}_{\mathbb{C}} \mathrm{a}^{\prime \prime}}{\epsilon} & \mathrm{e}_{\mathbb{C}}\left[-2\left(2+3 \mathrm{e}^{\prime \prime}\right)\left(1-3 \cos ^{2} \mathrm{I}^{\prime \prime}\right)+30 \mathrm{e}^{\prime \prime 2} \sin ^{2} \mathrm{I}^{\prime \prime} \cos 2 \mathrm{~g}^{\prime \prime}+6\left(2+3 \mathrm{e}^{\prime \prime 2}\right) \sin ^{2} \mathrm{I}^{\prime \prime} \cos 2 \mathrm{~h}^{\prime \prime}\right. \\
& \left.+15 \mathrm{e}^{\prime \prime 2}\left(1+\cos \mathrm{I}^{\prime \prime}\right)^{2} \cos \left(2 \mathrm{~g}^{\prime \prime}+2 \mathrm{~h}^{\prime \prime}\right)+15 \mathrm{e}^{\prime \prime 2}\left(1-\cos \mathrm{I}^{\prime \prime}\right)^{2} \cos \left(2 \mathrm{~g}^{\prime \prime}-2 \mathrm{~h}^{\prime \prime}\right)\right] \sin l_{\oplus} \cdot(106)
\end{aligned}
$$


It is worthwhile to note that, although this result is a part of a third-order generating function, its actual order is $\left(n_{\mathbb{C}} / n\right) e_{\mathbb{C}}$, which is considered to be a second-order quantity, because a small divisor $n_{\mathbb{C}}$ is introduced through the integration.

\section{THE INCLINATION OF THE MOON'S ORBIT TO ITS EQUATOR}

The expression for the earth's perturbation is

$$
\mathrm{n}_{\mathbb{C}}^{2} \mathrm{r}^{2} \mathrm{P}_{2}\left(\cos \mathrm{S}_{10}^{\prime}\right)
$$

where $\cos S_{10}^{\prime}=K\left(i_{\mathbb{C}}\right)$. By a Taylor expansion, if $\sin i_{\mathbb{C}}$ is small,

$$
\cos S_{10}^{\prime} \simeq K(0)+\left[\partial \frac{\partial\left(i_{\mathbb{C}}\right)}{\left.\partial \sin i_{\mathbb{C}}\right)}\right]_{i_{\mathbb{C}}=0} \sin i_{\mathbb{C}}=\cos \widetilde{S}_{10}^{\prime}+\left[\frac{\partial \cos S_{10}^{\prime}}{\cos i_{\mathbb{C}} \partial i_{\mathbb{C}}}\right]_{i_{\mathbb{C}}=0} \sin i_{\mathbb{C}} .
$$

Thus the correction to be introduced is

$$
F_{3}\left(i_{\mathbb{C}}\right)=\frac{3 n_{\mathbb{C}}^{2} r^{2}}{\epsilon}\left[\begin{array}{l}
\partial \cos S_{10}^{\prime} \\
\cos i_{\mathbb{C}}^{-} \partial i_{\mathbb{C}}
\end{array}\right]_{i_{\mathbb{C}}=0} \sin i_{\mathbb{C}} \cos \grave{S}_{10} .
$$

It is easily shown that the bracketed quantity in Equation 108 is

$$
\sin I \sin v_{\text {由 }} \sin (f+g) \text {. }
$$

Thus,

$$
F_{3}\left(i_{\mathbb{C}}\right)=3 \frac{n_{\mathbb{C}}^{2} r^{2}}{\epsilon} \cos \tilde{S}_{10}^{1} \sin (f+g) \sin v_{\oplus} \sin I \sin i_{\mathbb{C}}
$$

Now,

$$
\cos \widetilde{\mathrm{S}}_{10}^{\prime} \sin (\mathrm{f}+\mathrm{g})=\sin (\mathrm{f}+\mathrm{g}) \cos (\mathrm{f}+\mathrm{g}) \cos \mathrm{h}-\sin ^{2}(\mathrm{f}+\mathrm{g}) \sin \mathrm{h} \cos \mathrm{I}
$$

Therefore, the part of $F_{3}\left(i_{\mathbb{C}}\right)$ which is free from short-period terms is

$$
\begin{aligned}
F_{3}^{\prime}\left(i_{\mathbb{C}}\right)=\frac{3 n_{\mathbb{C}}^{2} a^{\prime 2}}{8 \epsilon} \sin v_{\oplus} \sin I^{\prime} & \sin i_{\mathbb{C}}\left[-2\left(2+3 e^{\prime 2}\right) \cos I^{\prime} \sin h^{\prime}\right. \\
& \left.+5 e^{\prime 2}\left(1+\cos I^{\prime}\right) \sin \left(2 g^{\prime}+h^{\prime}\right)+5 e^{\prime 2}\left(1-\cos I^{\prime}\right) \sin \left(2 g^{\prime}-h^{\prime}\right)\right] .
\end{aligned}
$$


There is no contribution to $\mathrm{F}_{3}^{\prime \prime}$ and $\mathrm{S}_{2}{ }^{\prime}$. The contribution to $\mathrm{S}_{3}{ }^{\prime}$ is

$S_{3}^{\prime}\left(i_{\mathscr{C}}\right)=-\frac{3 n_{\mathbb{C}}^{2} a^{\prime \prime 2}}{8 \varepsilon\left(n_{\mathbb{C}}+N_{\omega_{C}}\right)} \cos v_{\oplus} \sin I^{\prime} \sin i_{\mathscr{C}}\left[-2\left(2+3 e^{\prime \prime 2}\right) \cos I^{\prime \prime} \sin h^{\prime \prime}\right.$

$$
\left.+5 \mathrm{e}^{\prime \prime 2}\left(1+\cos \mathrm{I}^{\prime \prime}\right) \sin \left(2 \mathrm{~g}^{\prime \prime}+\mathrm{h}^{\prime \prime}\right)+5 \mathrm{e}^{\prime 2}\left(1-\cos \mathrm{I}^{\prime \prime}\right) \sin \left(2 \mathrm{~g}^{\prime \prime} \cdot \mathrm{h}^{\prime \prime}\right)\right]
$$

where $N_{\omega_{C}}$ is given by $\omega_{\mathbb{C}}=N_{\omega_{C}} t+$ const. Again, this is a second-order contribution, since $\mathbf{n}_{\mathbb{C}}^{2} /\left(\mathbf{n}_{\mathbb{C}}+N_{\omega_{C}}\right) \simeq n_{\mathbb{C}}$.

\section{THE NON-SPHERICITY OF THE POTENTIAL FIELD OF THE EARTH}

Because of the fact that the earth's equator is not the reference plane, the form of the disturbing function due to the zonal harmonic coefficient $j_{2}$ of the earth will be derived from basic relations.

In Figure 5, $\mathrm{E}$ is the earth, $\mathrm{M}$ the moon, and $\mathrm{S}$ the orbiter. The disturbing function for the motion of $\mathrm{S}$ is given by

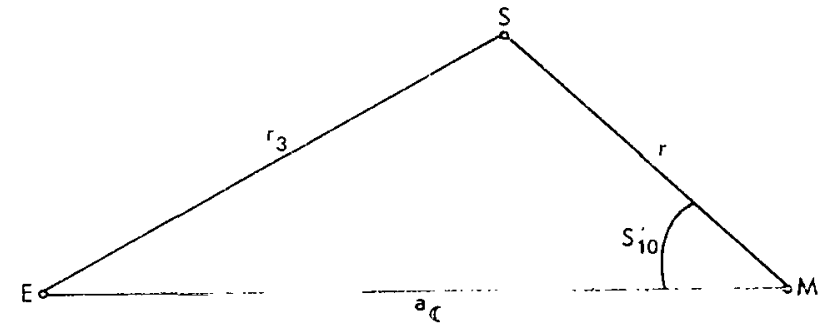

Figure 5-Earth-moon-orbiier configuration.

$$
F_{\bar{r}+1}=\frac{k^{2} m_{\oplus}}{r_{3}^{\prime}}\left[1-\frac{R_{\oplus}^{2} j_{2}}{r_{3}{ }^{2}} P_{2}(\sin \varphi)\right]
$$

where $R_{\oplus}$ is the equatorial radius of the earth and $\varphi$ the latitude of the orbiter with respect to the earth's equator. The part $\mathrm{k}^{2} \mathrm{~m}_{\oplus} / \mathrm{r}_{3}^{\prime}$ has already been taken into account, and the rest is a third-order quantity. Therefore,

$$
F_{3}(\uplus)--\frac{k^{2} m_{t r}}{r_{3}{ }^{3}} R_{\Psi}^{2} j_{2} P_{2}(\sin \Psi) \text {. }
$$

If the terms $\left(a / a_{\mathbb{C}}\right) j_{2}$ and $e_{\mathbb{C}} j_{2}$ are neglected, it follows that

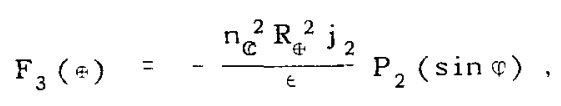

assuming $a_{\mathbb{C}} / r_{3}^{\prime}=1$. The angle $\psi$ must now be expressed in terms of the orbital elements referred to the lunar equator.

In Figure 6 the geometry of the problem is given. It follows that

$$
\sin \varphi=\left(\sin I \cos I_{\oplus}-\cos \psi \cos I \sin I_{\oplus}\right) \sin (f+g)-\sin I_{\oplus} \sin \psi \cos (f+g) .
$$


Then, if

$$
\overline{\mathrm{A}}=\sin \mathrm{I} \cos \mathrm{I}_{\oplus}-\cos \psi \cos \mathrm{I} \sin \mathrm{I}_{\oplus}
$$

and

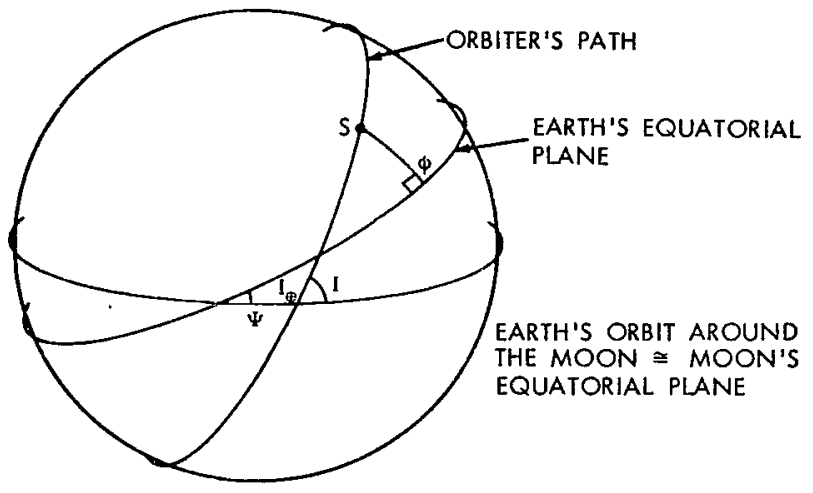

we have

$$
\overline{\mathrm{B}}=-\sin \mathrm{I}_{\oplus} \sin \psi,
$$

Figure 6-Planar configurations.

$$
F_{3}(\oplus)=-\frac{n_{C}^{2} R_{G}^{2} j_{2}}{\epsilon}\left[\frac{3}{4}\left(\bar{A}^{2}+\bar{B}^{2}\right)-\frac{1}{2}+\frac{3}{4}\left(\bar{B}^{2}-\bar{A}^{2}\right) \cos 2(f+g)+\frac{3}{2} \bar{A} \bar{B} \sin 2(f+g)\right] .
$$

Using the relation

$$
d l=d f+2 \sum_{k=1}^{\infty} k \cdot 3^{k}\left(\frac{1}{k}+\sqrt{1-e^{2}}\right) \cos k f(-1)^{k} d f
$$

where

$$
\beta=\frac{1}{e}\left(1-\sqrt{1-e^{2}}\right)
$$

elimination of short-period terms from Equation 115 yields

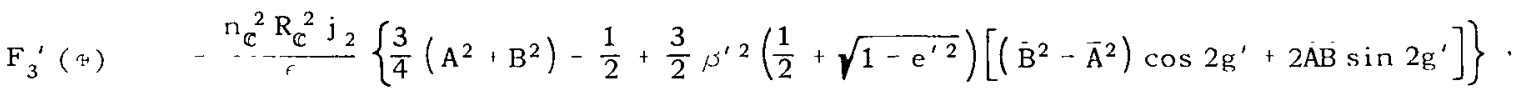

where

$$
i^{\prime}=3\left(\mathrm{e}^{\prime}\right)
$$

Furthermore, since $\psi \quad \mathrm{h}+\lambda_{\oplus}-\bar{\Omega}^{\prime}$, where $\bar{\Omega}^{\prime}$ is the longitude of the descending node of the moon's equator on the earth's equator, we have

$$
\begin{aligned}
\bar{B}^{2}+\bar{A}^{2}=\left(\sin ^{2} I^{\prime} \cos ^{2} I_{\oplus}+\frac{1}{2} \cos ^{2} I^{\prime} \sin ^{2} I_{\oplus}+\frac{1}{2} \sin ^{2} I_{\oplus}\right) & \\
-\frac{1}{2} \sin ^{2} I_{\oplus} \sin ^{2} I^{\prime} \cos 2\left(h^{\prime}+\lambda_{\oplus}-\bar{\Omega}^{\prime}\right) & -\frac{1}{2} \sin 2 I_{\oplus} \sin 2 I^{\prime} \cos \left(h^{\prime}+\lambda_{\oplus}-\bar{\Omega}^{\prime}\right)
\end{aligned}
$$




$$
\begin{aligned}
\overline{\mathrm{B}}^{2}-\overline{\mathrm{A}}^{2}=\left(\frac{1}{2} \sin ^{2} \mathrm{I}_{\oplus}-\sin ^{2} \mathrm{I}^{\prime} \cos ^{2} \mathrm{I}_{\oplus}-\frac{1}{2} \cos ^{2} \mathrm{I}^{\prime} \sin ^{2} \mathrm{I}_{\oplus}\right) & \\
& -\frac{1}{2} \sin ^{2} I_{\oplus}\left(1+\cos ^{2} \mathrm{I}^{\prime}\right) \cos 2\left(\mathrm{~h}^{\prime}+\lambda_{\oplus}-\bar{\Omega}^{\prime}\right) \\
& +\frac{1}{2} \sin 2 \mathrm{I}^{\prime} \sin 2 \mathrm{I}_{\oplus} \cos \left(\mathrm{h}^{\prime}+\lambda_{\oplus}-\bar{\Omega}^{\prime}\right),
\end{aligned}
$$

and

$$
\bar{A} \bar{B}=\frac{1}{2} \sin ^{2} I_{\oplus} \cos I^{\prime} \sin 2\left(h^{\prime}+\lambda_{\oplus}-\bar{\Omega}^{\prime}\right)-\frac{1}{2} \sin 2 I_{\oplus} \sin I^{\prime} \sin \left(h^{\prime}+\lambda_{\oplus}-\bar{\Omega}^{\prime}\right) .
$$

Thus the contribution to $S_{2}^{\prime}$ is zero, and

$$
\begin{aligned}
F_{3}^{\prime \prime}(\oplus)-\frac{1}{8} \frac{\mathrm{n}_{\mathbb{C}}^{2} \mathrm{j}_{2} R_{\oplus}^{2}}{\epsilon}\left[-\left(1-3 \cos ^{2} \mathrm{I}^{\prime \prime}\right)\right. \\
\left.+3 \xi^{\prime \prime \prime} 2\left(1+2 \sqrt{1-\mathrm{e}^{\prime \prime 2}}\right) \sin ^{2} \mathrm{I}^{\prime \prime} \cos 2 \mathrm{~g}^{\prime \prime}\right]\left(1-3 \cos ^{2} \mathrm{I}_{\oplus}\right) .
\end{aligned}
$$

The contribution to $S_{3}^{\prime}$ will be given by the integration of

$$
\begin{aligned}
& \frac{\partial S_{3}^{\prime}(\oplus)}{\partial \tau}=-\frac{3}{16} \frac{\mathrm{n}_{\mathbb{C}}{ }^{2} \mathrm{j}_{2} R_{\oplus}{ }^{2} \sin I_{\oplus}}{\epsilon}\left\{-2 \sin I^{\prime \prime}\left[\sin I^{\prime \prime} \sin I_{\oplus} \cos 2\left(h^{\prime}+\lambda_{\oplus}-\bar{\Omega}^{\prime}\right)+4 \cos I^{\prime \prime} \cos I_{\oplus} \cos \left(h^{\prime}+\lambda_{\oplus}-\bar{\Omega}^{\prime}\right)\right]\right.
\end{aligned}
$$

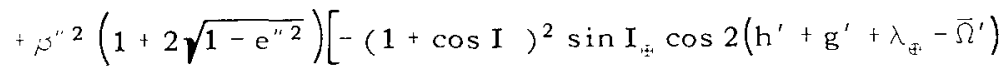

$$
\begin{aligned}
& -\left(1-\cos I^{\prime \prime}\right)^{2} \sin I_{e} \cos 2\left(h^{\prime}-g^{\prime}+\lambda_{\Psi}-\ddot{\Omega}^{\prime}\right)+4 \sin I^{\prime \prime}\left(1+\cos I^{\prime \prime}\right) \cos I_{\oplus} \cos \left(h^{\prime}+2 g^{\prime}+\lambda_{\Psi^{\prime}}-\bar{j}^{\prime}\right) \\
& \left.\left.-4 \sin I^{\prime}\left(1-\cos I^{\prime \prime}\right) \cos I_{\oplus} \cos \left(h^{\prime}-2 g^{\prime}+\lambda_{+}-\Sigma^{\prime}\right)\right]\right\}
\end{aligned}
$$

\section{Since}

$$
\frac{\partial}{\partial \tau}\left(\lambda_{\oplus}-\bar{\Omega}^{\prime}\right)=\mathrm{n}_{\mathbb{C}}^{*}-\mathrm{N}_{\Omega \mathfrak{C}}
$$

we have

$S_{3}^{\prime}(\oplus)=-\frac{3}{32} \frac{n_{\mathbb{C}}^{2} j_{2} R_{\oplus}^{2} \sin I_{\oplus}}{\epsilon\left(n_{\mathbb{C}}^{*}-N_{\Omega \mathbb{C}}\right)}\left\{-2 \sin I^{\prime \prime}\left[\sin I^{\prime \prime} \sin I_{\oplus} \sin 2\left(h^{\prime \prime}+\lambda_{\oplus}-\bar{\Omega}^{\prime}\right)+8 \cos I^{\prime \prime} \cos I_{\oplus} \sin \left(h^{\prime \prime}+\lambda_{\oplus}-\bar{\Omega}^{\prime}\right)\right]\right.$ 


$$
\begin{aligned}
& +\beta^{\prime \prime 2}\left(1+2 \sqrt{1-\mathrm{e}^{\prime \prime}}\right)\left[-\left(1+\cos \mathrm{I}^{\prime \prime}\right)^{2} \sin \mathrm{I}_{\oplus} \sin 2\left(\mathrm{~h}^{\prime \prime}+\mathrm{g}^{\prime \prime}+\lambda_{\oplus}-\bar{\Omega}^{\prime}\right)\right. \\
& -\left(1-\cos \mathrm{I}^{\prime \prime}\right)^{2} \sin \mathrm{I}_{\oplus} \sin 2\left(\mathrm{~h}^{\prime \prime}-\mathrm{g}^{\prime \prime}+\lambda_{\oplus}-\bar{\Omega}^{\prime}\right)+8 \sin \mathrm{I}^{\prime \prime}\left(1+\cos \mathrm{I}^{\prime \prime}\right) \cos \mathrm{I}_{\oplus} \sin \left(\mathrm{h}^{\prime \prime}+2 \mathrm{~g}^{\prime \prime}+\lambda_{\oplus}-\bar{\Omega}^{\prime}\right) \\
& \left.\left.-8 \sin \mathrm{I}^{\prime \prime}\left(1-\cos \mathrm{I}^{\prime \prime}\right) \cos \mathrm{I}_{\oplus} \sin \left(\mathrm{h}^{\prime \prime}-2 \mathrm{~g}^{\prime \prime}+\lambda_{\oplus}-\bar{\Omega}^{\prime}\right)\right]\right\} \cdot(1
\end{aligned}
$$

\section{PHYSICAL LIBRATION, AND THE PRECESSION OF THE LUNAR EQUATOR}

Physical libration causes a periodic oscillation in the position of the lunar surface, thereby creating a small angular displacement between the principal lunar meridian and the earth-moon line of centers. The largest contribution to this displacement is given by

$$
x=a \sin \ell_{\odot}+x_{0} \quad \text { (see Reference 4, p. 316) }
$$

where

$$
\begin{aligned}
a & =-59^{\prime \prime}=-2.86 \times 10^{-4} \mathrm{rad} \\
x_{0} & =\text { constant dependent upon the initial time, }
\end{aligned}
$$

and

$$
l_{\Theta}=\text { mean anomaly of the sun. }
$$

Let $s \eta \zeta$ and $x y z$ be selenocentric, equatorial coordinate systems with the $\xi$-axis directed toward the earth and the $x$-axis passing through the principal meridian (see Figure 7). Then,

$$
\begin{aligned}
& \ddot{\xi}=\frac{\partial \mathrm{F}}{\partial \xi}, \quad \ddot{\eta}=\frac{\partial \mathrm{F}}{\partial \eta}, \\
& \mathbf{x}=\xi \cos x+\eta \sin x,
\end{aligned}
$$

and

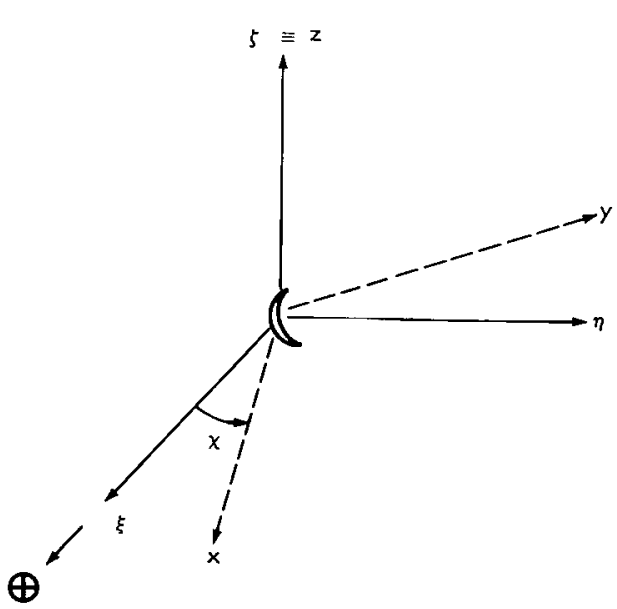

Figure 7-Selenocentric, equatorial coordinate systems. 
Thus,

$\ddot{x}=\ddot{\xi} \cos x+\ddot{\eta} \sin x+2 \alpha n_{\odot} \cos l_{\odot}(\dot{\eta} \cos x-\dot{\xi} \sin \chi)$

$$
-a{ }_{\odot}^{2} \sin \ell_{\odot}(\eta \cos x-\xi \sin \chi)-a^{2} n_{\odot}^{2} \cos ^{2} l_{\odot}(\eta \sin x+\xi \cos x)
$$

where $n_{\odot}=$ mean motion of the sun's mean anomaly. But

$$
\dot{n} \cos x-\dot{\xi} \sin \chi=\operatorname{an}_{\odot} x \cos \ell_{\odot}+\dot{y}
$$

and

$$
\ddot{\xi} \cos x+\ddot{\eta} \sin x=\frac{\partial F}{\partial x} \text {, }
$$

so Equation 122 becomes

$$
\ddot{x}=\frac{\partial F}{\partial x}+2 \alpha n_{\odot} \cos l_{\odot}\left(a n_{\odot} x \cos \ell_{\odot}+\dot{y}\right)-\alpha n_{\odot}^{2} \sin \ell_{\odot}(\eta \cos x-\xi \sin x)-\alpha^{2} n_{\odot}^{2} x \cos ^{2} \ell_{\odot} .
$$

If one neglects terms higher than third-order, Equation 124 reduces to

$$
\ddot{x}=\frac{\partial F}{\partial x}+2 a n_{\odot} \dot{y} \cos \ell_{\odot} \text {. }
$$

A similar computation yields

$$
\ddot{y}=\frac{\partial F}{\partial y}-2 a n_{\odot} \dot{x} \cos \ell_{\odot} \text {. }
$$

Thus,

$$
\ddot{x}=\frac{\partial \overline{\mathrm{F}}}{\partial \mathrm{x}}, \quad \ddot{\mathrm{y}}=\frac{\partial \overline{\mathrm{F}}}{\partial \mathrm{y}} \text {. }
$$

where

$$
\begin{aligned}
\bar{F} & =F+2 a n_{\odot}(x \dot{y}-\dot{x} y) \cos \ell_{\odot} \\
& =F+2 a n_{\odot} H \cos \ell_{\odot} .
\end{aligned}
$$


Hence, the addition to the Hamiltonian of the problem is

$$
F_{3}(1 \mathrm{ib} .)=2 a n_{\odot} H \cos \ell_{\odot} .
$$

Then,

$$
\begin{aligned}
& F_{3 \mathrm{~s}}(1 \mathrm{ib} .) \equiv 0 \\
& \mathrm{~F}_{3 \mathrm{ph}} \cdot(1 \mathrm{ib} .) \equiv 0 \\
& F_{3 \mathrm{p} \tau}(1 \mathrm{ib} .)=2 a \mathrm{n}_{\odot} H \cos \ell_{\odot},
\end{aligned}
$$

and therefore

$$
\frac{\partial \mathrm{S}_{3}{ }^{\prime}(1 \mathrm{ib} .)}{\partial \tau^{-}}=2 a \mathrm{n}_{\odot} \mathrm{H} \cos \ell_{\odot} \Longrightarrow \mathrm{S}_{3}{ }^{\prime}(1 \mathrm{ib} .)=2 a \mathrm{H} \sin \ell_{\odot} .
$$

Now, the precession in the lunar equator is created by the small angle of inclination $\left(\sim 1^{\circ} 32: 1\right)$ between the lunar equatorial plane and the ecliptic. This results in the regression of the equatorial node in the ecliptic. However, the motion of the node is already implicit in the $n_{\mathbb{C}}^{*}-$ hence no additional corrections need be made.

\section{COMPLETE "SECULAR" THIRD-ORDER HAMILTONIAN}

The complete secular third-order Hamiltonian is given by

$$
\mathrm{F}_{3}^{\prime \prime}=\mathrm{F}_{3}^{\prime \prime}(\operatorname{coupl} \text { ing })+\mathrm{F}_{3}^{\prime \prime}(\operatorname{sun})+\mathrm{F}_{3}^{\prime \prime}(\oplus) \text {, }
$$

where $F_{3}^{\prime \prime}$ (coupling) is given by Equation 86, $F_{3}^{\prime \prime}$ (sun) by Equation 96, and $F_{3}^{\prime \prime}(\oplus)$ by Equation 117.

\section{THE DETERMINING FUNCTION FOR LONG-PERIOD TERMS}

Additional long-period perturbations depending on the motion of the node, the motion of the earth, and the motion of the sun will be given through the determining functions $S_{2}{ }^{\prime}$ and $S_{3}{ }^{\prime}$. The determining functions $\mathrm{S}_{2}{ }^{\prime}$ and $\mathrm{s}_{3}{ }^{\prime}$ are

$$
S_{2}^{\prime}=S_{2}^{\prime} \text { (coupling) }+S_{2}^{\prime}(\text { earth })
$$


and

$$
S_{3}^{\prime}=S_{3}^{\prime} \text { (radiation) }+S_{3}^{\prime}(\text { sun })+S_{3}^{\prime}\left(e_{\mathbb{C}}\right)+S_{3}^{\prime}\left(i_{\mathbb{C}}\right)+S_{3}^{\prime}(\oplus)+S_{3}^{\prime} \text { (1ibration); }
$$

the various functions are defined by Equations 85, 92, 97, 102, 106, 111, 119, and 131.

In the next section, the partial derivatives needed in order to find additional perturbations in the canonical elements are given.

\section{LONG-PERIOD PERTURBATIONS OF SECOND ORDER}

The perturbations of long period are obtained from

$$
\begin{array}{ll}
l^{\prime \prime}=\frac{\partial \mathrm{S}}{\partial \mathrm{L}^{\prime \prime}}=l^{\prime}+\frac{\partial \mathrm{S}_{1}^{\prime}}{\partial \mathrm{L}^{\prime \prime}}+\frac{\partial \mathrm{S}_{2}^{\prime}}{\partial \mathrm{L}^{\prime \prime}}+\frac{\partial \mathrm{S}_{3}^{\prime}}{\partial \mathrm{L}^{\prime \prime}}, & \mathrm{L}^{\prime}=\frac{\partial \mathrm{S}}{\partial l^{\prime}}=\mathrm{L}^{\prime \prime}, \\
\mathrm{g}^{\prime \prime}=\frac{\partial \mathrm{S}}{\partial \mathrm{G}^{\prime \prime}}=\mathrm{g}^{\prime}+\frac{\partial \mathrm{S}_{1}^{\prime}}{\partial \mathrm{G}^{\prime \prime}}+\frac{\partial \mathrm{S}_{2}^{\prime}}{\partial \mathrm{G}^{\prime \prime}}+\frac{\partial \mathrm{S}_{3}^{\prime}}{\partial \mathrm{G}^{\prime \prime}}, & \mathrm{G}^{\prime}=\frac{\partial \mathrm{S}}{\partial \mathrm{g}^{\prime}}=\mathrm{G}^{\prime \prime}+\frac{\partial \mathrm{S}_{1}^{\prime}}{\partial \mathrm{g}^{\prime}}+\frac{\partial \mathrm{S}_{2}^{\prime}}{\partial \mathrm{g}^{\prime}}+\frac{\partial \mathrm{S}_{3}{ }^{\prime}}{\partial \mathrm{g}^{\prime}}, \\
\mathrm{h}^{\prime \prime}=\frac{\partial \mathrm{S}}{\partial \mathrm{H}^{\prime \prime}}=\mathrm{h}^{\prime}+\frac{\partial \mathrm{S}_{1}^{\prime}}{\partial \mathrm{H}^{\prime \prime}}+\frac{\partial \mathrm{S}_{2}^{\prime}}{\partial \mathrm{H}^{\prime \prime}}+\frac{\partial \mathrm{S}_{3}^{\prime}}{\partial \mathrm{H}^{\prime \prime}}, & \mathrm{H}^{\prime}=\frac{\partial \mathrm{S}}{\partial \mathrm{h}^{\prime}}=\mathrm{H}^{\prime \prime}+\frac{\partial \mathrm{S}_{1}^{\prime}}{\partial \mathrm{h}^{\prime}}+\frac{\partial \mathrm{S}_{2}^{\prime}}{\partial \mathrm{h}^{\prime}}+\frac{\partial \mathrm{S}_{3}^{\prime}}{\partial \mathrm{h}^{\prime}} .
\end{array}
$$

The terms corresponding to $S_{1}^{\prime}$ have already been obtained. Next the partial derivatives of $S_{2}^{\prime}$ and $S_{3}^{\prime}$ with respect to $a^{\prime \prime}, e^{\prime \prime}, I^{\prime \prime}, g^{\prime}$, and $h^{\prime}$ are computed. For convenience, however, the primes have been dropped in this section.

The "mean" mean motion $n$ ' (called $n$ ) depends on $a^{\prime}$ (called a) through the relation

$$
n^{2}=\frac{\mu}{a^{3}},
$$

and

$$
\begin{aligned}
& \frac{\partial \mathrm{S}}{\partial \mathrm{L}}=2 \sqrt{\frac{\mathrm{a}}{\mu} \frac{\partial \mathrm{S}}{\partial \mathrm{a}}+\frac{1-\mathrm{e}^{2}}{\mathrm{e} \sqrt{\mu \mathrm{a}}} \frac{\partial \mathrm{S}}{\partial \mathrm{e}},} \\
& \frac{\partial \mathrm{S}}{\partial \mathrm{G}}=-\frac{1}{\mathrm{e}} \sqrt{\frac{1-\mathrm{e}^{2}}{\mu \mathrm{a}}} \frac{\partial \mathrm{S}}{\partial \mathrm{e}}+\frac{\cot \mathrm{I}}{\sqrt{\mu \mathrm{a}\left(1-\mathrm{e}^{2}\right)}} \frac{\partial \mathrm{S}}{\partial \mathrm{I}},
\end{aligned}
$$


and

$$
\frac{\partial \mathrm{S}}{\partial \mathrm{H}}=-\sin \frac{1}{\sqrt{\mu \mathrm{a}\left(1-\mathrm{e}^{2}\right)}} \frac{\partial \mathrm{S}}{\partial \mathbf{I}} .
$$

The following definitions are made:

$$
\begin{aligned}
& C_{1}=\left(1-e^{2}\right)\left(2-17 e^{2}\right) \sin ^{2} I \cos I \\
& C_{2}=\left(2+3 e^{2}\right)^{2} \sin ^{2} I \cos I \\
& C_{3}=e^{2}\left(1-e^{2}\right)(1+\cos I)^{2}(2-3 \cos I) \\
& C_{4}=e^{2}\left(1-e^{2}\right)(1-\cos I)^{2}(2+3 \cos I) \\
& C_{5}=e^{2} \sin ^{2} I(1+\cos I)\left[6 e^{2}-(1+5 \cos I)\right] \\
& C_{6}=e^{2} \sin ^{2} I(1-\cos I)\left[6 e^{2}-(1-5 \cos I)\right] \\
& C_{7}=e^{2}(1+\cos I)^{3}\left[2 e^{2}-(1+\cos I)\right] \\
& C_{8}=e^{2}(1-\cos I)^{3}\left[2 e^{2}-(1-\cos I)\right] \\
& C_{9}=\left(2+3 e^{2}\right) \sin ^{2} I \cos I \\
& C_{10}=e^{2}(1+\cos I)^{2}\left(1+2 \cos I-5 \cos ^{2} I\right) \\
& C_{11}=e^{2}(1-\cos I)^{2}\left(1-2 \cos I-5 \cos ^{2} I\right) \\
& C_{12}=\left(2+3 e^{2}\right) \cos I \\
& C_{13}=e^{2}(1+\cos I)(3-5 \cos I) \\
& C_{14}=e^{2}(1-\cos I)(3+5 \cos I) \\
& C_{15}=e^{2}(1+\cos I)(1+5 \cos I) \\
& C_{16}=e^{2}(1-\cos I)(1-5 \cos I) \\
& C_{17}=(1+\cos I)\left[25\left(1-\cos I-\cos ^{2} I-7 \cos ^{3} I\right)-\left(1-e^{2}\right)\left(9+15 \cos I+15 \cos ^{2} I-175 \cos ^{3} I\right)\right] \\
& C_{18}{ }^{-}=(1-\cos I)\left[25\left(1+\cos I-\cos ^{2} I+7 \cos ^{3} I\right)-\left(1-e^{2}\right)\left(9-15 \cos I+15 \cos ^{2} I+175 \cos ^{3} I\right)\right]
\end{aligned}
$$




$$
\begin{aligned}
& C_{19}=e^{2}(1+\cos I)^{2}\left(1+2 \cos I-7 \cos ^{2} I\right) \\
& C_{20}=e^{2}(1-\cos I)^{2}\left(1-2 \cos I-7 \cos ^{2} I\right) \\
& C_{21}=(1+\cos I)\left(3-25 \cos I+5 \cos ^{2} I+25 \cos ^{3} I\right) \\
& C_{22}=(1-\cos I)\left(3+25 \cos I+5 \cos ^{2} I-25 \cos ^{3} I\right) \\
& C_{23}=\sin ^{2} I \cos I\left[35-10\left(1-e^{2}\right)\left(2+7 \cos ^{2} I\right)-3\left(1-e^{2}\right)^{2}\left(1-14 \cos ^{2} I\right)\right] \\
& C_{24}=e^{2}(1+\cos I)^{2}\left[35\left(3+4 \cos I-30 \cos ^{2} I-20 \cos ^{3} I+51 \cos ^{4} I\right)\right. \\
& \left.-3\left(1-e^{2}\right)\left(11+36 \cos I-126 \cos ^{2} I-196 \cos ^{3} I+315 \cos ^{4} I\right)\right] \\
& C_{25}=e^{2}(1-\cos I)^{2}\left[35\left(3-4 \cos I-30 \cos ^{2} I+20 \cos ^{3} I+51 \cos ^{4} I\right)\right. \\
& \left.-3\left(1-e^{2}\right)\left(11-36 \cos I-126 \cos ^{2} I+196 \cos ^{3} I+315 \cos ^{4} I\right)\right] \\
& C_{26}=e^{4} \sin ^{2} I(1+\cos I)^{2}\left(1+2 \cos I-9 \cos ^{2} I\right) \\
& C_{27}=e^{4} \sin ^{2} I(1-\cos I)^{2}\left(1-2 \cos I-9 \cos ^{2} I\right) \\
& C_{28}=\left(2+3 e^{2}\right) \cos I\left(3-7 \cos ^{2} I\right) \\
& C_{29}=e^{2}(1+\cos I)\left(3-19 \cos I-7 \cos ^{2} I+35 \cos ^{3} I\right) \\
& C_{30}=e^{2}(1-\cos I)\left(3+19 \cos I-7 \cos ^{2} I-35 \cos ^{3} I\right) \\
& C_{31}=(1+\cos I)\left[35\left(15+41 \cos I-262 \cos ^{2} I-346 \cos ^{3} I+519 \cos ^{4} I+321 \cos ^{5} I\right)\right. \\
& +14\left(1-e^{2}\right)\left(259-255 \cos I-566 \cos ^{2} I+1710 \cos ^{3} I-525 \cos ^{4} I-1455 \cos ^{5} I\right) \\
& \left.-\left(1-e^{2}\right)^{2}\left(1559-1015 \cos I-5446 \cos ^{2} I+7350 \cos ^{3} I+735 \cos ^{4} I-5775 \cos ^{5} I\right)\right] \\
& C_{32}=(1-\cos I)\left[35\left(15-41 \cos I-262 \cos ^{2} I+346 \cos ^{3} I+519 \cos ^{4} I-321 \cos ^{5} I\right)\right. \\
& +14\left(1-\mathrm{e}^{2}\right)\left(259+255 \cos \mathrm{I}-566 \cos ^{2} \mathrm{I}-1710 \cos ^{3} \mathrm{I}-525 \cos ^{4} \mathrm{I}+1455 \cos ^{5} \mathrm{I}\right) \\
& \left.-\left(1-\mathrm{e}^{2}\right)^{2}\left(1559+1015 \cos \mathrm{I}-5446 \cos ^{2} \mathrm{I}-7350 \cos ^{3} \mathrm{I}+735 \cos ^{4} \mathrm{I}+5775 \cos ^{5} \mathrm{I}\right)\right]
\end{aligned}
$$




$$
\begin{aligned}
& C_{33}=(1+\cos I)^{2}\left[5\left(9-7 \cos I-119 \cos ^{2} I+15 \cos ^{3} I+174 \cos ^{4} I\right)\right. \\
& -\left(1-e^{2}\right)\left(179-14 \cos I-1320 \cos ^{2} I+30 \cos ^{3} I+1605 \cos ^{4} I\right) \\
& \left.+3\left(1-e^{2}\right)^{2}\left(18+7 \cos I-135 \cos ^{2} I-15 \cos ^{3} I+165 \cos ^{4} I\right)\right] \\
& C_{34}=(1-\cos I)^{2}\left[5\left(9+7 \cos I-119 \cos ^{2} I-15 \cos ^{3} I+174 \cos ^{4} I\right)\right. \\
& -\left(1-e^{2}\right)\left(179+14 \cos I-1320 \cos ^{2} I-30 \cos ^{3} I+1605 \cos ^{4} I\right) \\
& \left.+3\left(1-e^{2}\right)^{2}\left(18-7 \cos I-135 \cos ^{2} I+15 \cos ^{3} I+165 \cos ^{4} I\right)\right] \\
& C_{35}=e^{2} \sin ^{2} I(1+\cos I)^{2}\left[1-9 \cos ^{2} I-\left(1-e^{2}\right)\left(3-11 \cos ^{2} I\right)\right] \\
& C_{36}=e^{2} \sin ^{2} I(1-\cos I)^{2}\left[1-9 \cos ^{2} I-\left(1-e^{2}\right)\left(3-11 \cos ^{2} I\right)\right] \\
& C_{37}=\left(4+3 e^{2}\right)(1+\cos I)(3-5 \cos I)\left(29-126 \cos ^{2} I+105 \cos ^{4} I\right) \\
& C_{38}=\left(4+3 e^{2}\right)(1-\cos I)(3+5 \cos I)\left(29-126 \cos ^{2} I+105 \cos ^{4} I\right) \\
& C_{39}=e^{2} \sin ^{2} I(1+\cos I)(3-5 \cos I)\left(7-15 \cos ^{2} I\right) \\
& C_{40}=e^{2} \sin ^{2} I(1-\cos I)(3+5 \cos I)\left(7-15 \cos ^{2} I\right)
\end{aligned}
$$

Then, the partial derivatives are as follows:

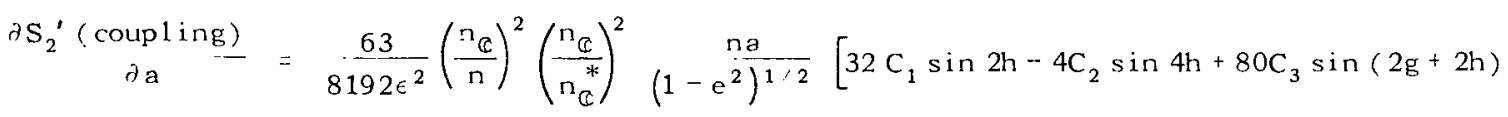

$$
\begin{aligned}
& \left.+80 C_{4} \sin (2 g-2 h)+10 C_{5} \sin (2 g+4 h)+10 C_{6} \sin (2 g-4 h)+25 C_{7} \sin (4 g+4 h)+25 C_{8} \sin (4 g-4 h)\right] \\
& -\frac{27}{512 \epsilon}\left(\frac{n_{\overparen{C}}}{n_{C}^{*}}\right)^{2} J_{2} b^{2} \quad \frac{n}{a\left(1-e^{2}\right)^{2}}\left[4 C_{9} \sin 2 h+5 C_{10} \sin (2 g+2 h)+5 C_{11} \sin (2 g-2 h)\right] \\
& +\frac{27}{1024 \epsilon}\left(\frac{n_{C}}{n_{C}^{*}}\right)^{2} J_{22} b^{2} \frac{n \sin ^{2} I}{a\left(1-e^{2}\right)^{2}}\left[-8 C_{12}(2 \sin 2 h-\sin 4 h)-20 C_{13} \sin (2 g+2 h)\right. \\
& \left.-20 C_{14} \sin (2 g-2 h)+5 C_{15} \sin (2 g+4 h)+5 C_{16} \sin (2 g-4 h)\right]
\end{aligned}
$$




$$
\begin{aligned}
& -\frac{117}{16}\left(\frac{n}{n_{\mathbb{C}}^{*}}\right)^{2} J_{2} J_{22} b^{4} \frac{n \sin ^{2} I \cos I}{a^{3}\left(1-e^{2}\right)^{7 / 2}} \sin 2 h+\frac{117}{32}\left(\frac{n}{n_{\mathbb{C}}^{*}}\right)^{2} J_{22}^{2} b^{4} \frac{n \sin ^{2} I \cos I}{a^{3}\left(1-e^{2}\right)^{7 / 2}} \sin 4 h \\
& -\frac{45}{2048 \epsilon}\left(\frac{n_{C}}{n_{C}^{*}}\right)^{2} J_{3} b^{3} \frac{n e \sin I}{a^{2}\left(1-e^{2}\right)^{3}}\left[C_{17} \cos (g+2 h)+C_{18} \cos (g-2 h)-25 C_{19} \cos (3 g+2 h)-25 C_{20} \cos (3 g-2 h)\right] \\
& -\frac{135}{256}\left(\frac{\mathrm{n}}{\mathrm{n}_{\mathscr{C}}^{*}}\right)^{2} J_{3} J_{22} b^{5} \frac{n e \sin I}{a^{4}\left(1-e^{2}\right)^{9 / 2}}\left[C_{21} \cos (g+2 h)+C_{22} \cos (g-2 h)\right] \\
& -\frac{315}{16384 \epsilon}\left(\frac{n_{\mathbb{C}}}{n_{\mathbb{C}}^{*}}\right)^{2} J_{4} b^{4} \frac{n}{a^{3}\left(1-e^{2}\right)^{4}}\left[8 C_{23} \sin 2 h+C_{24} \sin (2 g+2 h)+C_{25} \sin (2 g-2 h)\right. \\
& \left.-35 C_{26} \sin (4 g+2 h)-35 C_{27} \sin (4 g-2 h)\right] \\
& -\frac{765}{1024}\left(\frac{n}{n_{C}^{*}}\right)^{2} J_{4} J_{22} b^{6} \frac{n \sin ^{2} I}{a^{5}\left(1-e^{2}\right)^{11 / 2}}\left[4 C_{28} \sin 2 h+C_{29} \sin (2 g+2 h)+C_{30} \sin (2 g-2 h)\right] \\
& -\frac{405}{65536 \epsilon}\left(\frac{n_{\mathbb{C}}}{n_{\mathbb{C}}^{*}}\right)^{2} J_{5} b^{5}-\frac{n e s i n I}{a^{4}\left(1-e^{2}\right)^{5}}\left[C_{31} \cos (g+2 h)+C_{32} \cos (g-2 h)-14 C_{33} \cos (3 g+2 h)\right. \\
& \left.-14 C_{34} \cos (3 g-2 h)+105 C_{35} \cos (5 g+2 h)+105 C_{36} \cos (5 g-2 h)\right] \\
& -\frac{855}{8192}\left(\frac{\mathrm{n}}{\mathrm{n}^{*}{ }^{*}}\right)^{2} J_{5} J_{22} b^{7} \frac{n e \sin I}{a^{6}\left(1-e^{2}\right)^{13} i_{2}}\left[2 C_{37} \cos (g+2 h)+2 C_{38} \cos (g-2 h)\right. \\
& \left.-7 C_{39} \cos (3 g+2 h)-7 C_{40} \cos (3 g-2 h)\right] \\
& \underbrace{\partial S_{2}^{\prime} \text { ( coupling) }}_{2}=\frac{9}{2048 \epsilon^{2}}\left(\frac{n_{\overparen{C}}}{n}\right)^{2}\left(\frac{n_{\overparen{C}}}{n_{\overparen{C}}^{*}}\right)^{2} \frac{n a^{2} \sin I}{\sqrt{1-e^{2}}}\left\{-16\left(1-3 \cos ^{2} I\right)\left(1-e^{2}\right)\left(2-17 e^{2}\right) \sin 2 h\right. \\
& +2\left(1-3 \cos ^{2} I\right)\left(2+3 e^{2}\right)^{2} \sin 4 h-40(1+\cos I)(1-9 \cos I) e^{2}\left(1-e^{2}\right) \sin (2 g+2 h) \\
& +40(1-\cos I)(1+9 \cos I) e^{2}\left(1-e^{2}\right) \sin (2 g-2 h)+10(1+\cos I) e^{2}[(3-5 \cos I)(1+2 \cos I) \\
& \left.-3 e^{2}(1-3 \cos I)\right] \sin (2 g+4 h)-10(1-\cos I) e^{2}[(3+5 \cos I)(1-2 \cos I)
\end{aligned}
$$




$$
\begin{aligned}
& \left.-3 e^{2}(1+3 \cos I)\right] \sin (2 g-4 h)+25(1+\cos I)^{2} e^{2}\left[2(1+\cos I)-3 e^{2}\right] \sin (4 g+4 h) \\
& \left.-25(1-\cos I)^{2} e^{2}\left[2(1-\cos I)-3 e^{2}\right] \sin (4 g-4 h)\right\} \\
& +\frac{9}{64 \epsilon}\left(\frac{n_{\overparen{C}}}{n_{\overparen{C}}^{*}}\right)^{2} J_{2} b^{2} \frac{n \sin I}{\left(1-e^{2}\right)^{2}}\left[-\left(1-3 \cos ^{2} I\right)\left(2+3 e^{2}\right) \sin 2 h\right. \\
& \left.-5(1+\cos I)\left(1-\cos I-5 \cos ^{2} I\right) e^{2} \sin (2 g+2 h)+5(1-\cos I)\left(1+\cos I-5 \cos ^{2} I\right) e^{2} \sin (2 g-2 h)\right] \\
& -\frac{9}{256 \epsilon}\left(\frac{n_{C}}{n_{C}^{*}}\right)^{2} J_{22} b^{2} \frac{n \sin I}{\left(1-e^{2}\right)^{2}}\left[4\left(1-3 \cos ^{2} I\right)\left(2+3 e^{2}\right)(2 \sin 2 h-\sin 4 h)\right. \\
& -20(1+\cos I)\left(I+7 \cos I-10 \cos ^{2} I\right) e^{2} \sin (2 g+2 h) \\
& +20(1-\cos I)\left(1-7 \cos I-10 \cos ^{2} I\right) e^{2} \sin (2 g-2 h) \\
& -5(1+\cos I)(1+2 \cos I)(3-5 \cos I) e^{2} \sin (2 g+4 h)
\end{aligned}
$$$$
\left.+5(1-\cos I)(1-2 \cos I)(3+5 \cos I) e^{2} \sin (2 g-4 h)\right]
$$

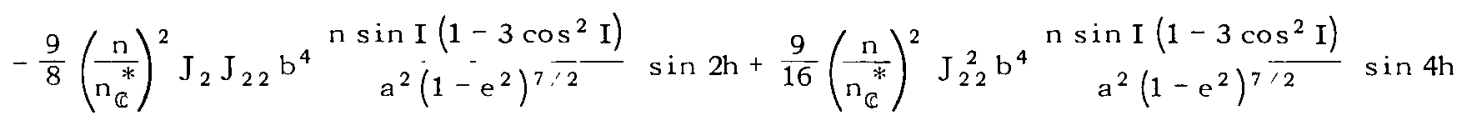

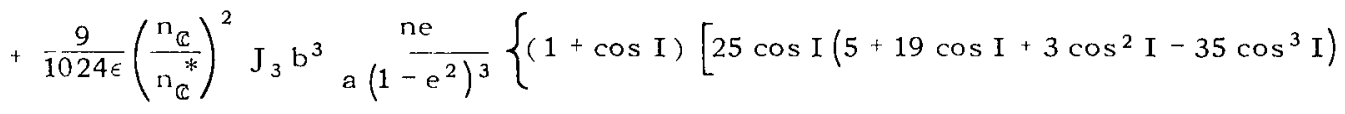$$
\left.+\left(1-\mathrm{e}^{2}\right)\left(24+27 \cos \mathrm{I}-555 \cos ^{2} \mathrm{I}-235 \cos ^{3} \mathrm{I}+875 \cos ^{4} \mathrm{I}\right)\right] \cos (\mathrm{g}+2 \mathrm{~h})
$$$$
+(1-\cos I)\left[25 \cos I\left(5-19 \cos I+3 \cos ^{2} I+35 \cos ^{3} I\right)\right.
$$$$
\left.-\left(1-e^{2}\right)\left(24-27 \cos I-555 \cos ^{2} I+235 \cos ^{3} I+875 \cos ^{4} I\right)\right] \cos (g-2 h)
$$$$
+25 e^{2}(1+\cos I)^{2}\left(4-13 \cos I-22 \cos ^{2} I+35 \cos ^{3} I\right) \cos (3 g+2 h)
$$$$
\left.-25 e^{2}(1-\cos I)^{2}\left(4+13 \cos I-22 \cos ^{2} I-35 \cos ^{3} I\right) \cos (3 g-2 h)\right\}
$$ 


$$
\begin{aligned}
& +\frac{9}{128}\left(\frac{n}{n_{\overparen{C}}^{*}}\right)^{2} J_{3} J_{22} b^{5} \frac{n e}{a^{3}\left(1-e^{2}\right)^{9 / 2}}\left[(1+\cos I)\left(22+21 \cos I-155 \cos ^{2} I-5 \cos ^{3} I+125 \cos ^{4} I\right) \cos ^{2}(g+2 h)\right. \\
& \left.-(1-\cos I)\left(22-21 \cos I-155 \cos ^{2} I+5 \cos ^{3} I+125 \cos ^{4} I\right) \cos (g-2 h)\right] \\
& -\frac{45}{4096 \epsilon}\left(\frac{n_{\mathbb{C}}}{n_{\mathbb{C}}^{*}}\right)^{2} J_{4} b^{4} \frac{n \sin I}{a^{2}\left(1-e^{2}\right)^{4}}\left\{4 \left[35\left(1-3 \cos ^{2} I\right)-10\left(1-e^{2}\right)\left(2+15 \cos ^{2} I-35 \cos ^{4} I\right)\right.\right. \\
& \left.-3\left(1-e^{2}\right)^{2}\left(1-45 \cos ^{2} I+70 \cos ^{4} I\right)\right] \sin 2 h+e^{2}(1+\cos I)\left[35\left(5-24 \cos I-90 \cos ^{2} I+52 \cos ^{3} I+153 \cos ^{4} I\right)\right. \\
& \left.-3\left(1-e^{2}\right)\left(29-72 \cos I-546 \cos ^{2} I+140 \cos ^{3} I+945 \cos ^{4} I\right)\right] \sin (2 g+2 h) \\
& -e^{2}(1-\cos I)\left[35\left(5+24 \cos I-90 \cos ^{2} I-52 \cos ^{3} I+153 \cos ^{4} I\right)\right. \\
& \left.-3\left(1-e^{2}\right)\left(29+72 \cos I-546 \cos ^{2} I-140 \cos ^{3} I+945 \cos ^{4} I\right)\right] \sin (2 g-2 h) \\
& -35 e^{4}\left[(1+\cos I)^{2}\left(2-9 \cos I-14 \cos ^{2} I+27 \cos ^{3} I\right) \sin (4 g+2 h)\right. \\
& \left.\left.-(1-\cos I)^{2}\left(2+9 \cos I-14 \cos ^{2} I+27 \cos ^{3} I\right) \sin (4 g-2 h)\right]\right\} \\
& -\frac{45}{512}\left(\frac{n}{n_{C}^{*}}\right)^{2} J_{4} J_{22} b^{6} \frac{n \sin I}{a^{4}\left(1-e^{2}\right)^{1 / 2}}\left[4\left(2+3 e^{2}\right)\left(3-30 \cos ^{2} I+35 \cos ^{4} I\right) \sin 2 h\right. \\
& -2 e^{2}(1+\cos I)\left(8+21 \cos I-87 \cos ^{2} I-35 \cos ^{3} I+105 \cos ^{4} I\right) \sin (2 g+2 h) \\
& \left.+2 e^{2}(1-\cos I)\left(8-21 \cos I-87 \cos ^{2} I+35 \cos ^{3} I+105 \cos ^{4} I\right) \sin (2 g-2 h)\right] \\
& +\frac{45}{32768 \epsilon}\left(\frac{n_{\overparen{C}}}{n_{C}^{*}}\right)^{2} J_{5} b^{5} \frac{n e}{a^{3}\left(1-e^{2}\right)^{5}}\left\{-(1+\cos I)\left[3 5 \left(56-513 \cos I-1423 \cos ^{2} I+2778 \cos ^{3} I+3854 \cos ^{4} I\right.\right.\right. \\
& \left.-2793 \cos ^{5} I-2247 \cos ^{6} I\right)+14\left(1-e^{2}\right)\left(4-1905 \cos I+5329 \cos ^{2} I+1874 \cos ^{3} I\right. \\
& \left.-16350 \cos ^{4} I+1695 \cos ^{5} I+10185 \cos ^{6} I\right)-\left(1-e^{2}\right)^{2}\left(544-15025 \cos I+19649 \cos ^{2} I\right. \\
& \left.\left.+32074 \cos ^{3} I-64890 \cos ^{4} I-10185 \cos ^{5} I+40425 \cos ^{6} I\right)\right] \cos (g+2 h)
\end{aligned}
$$




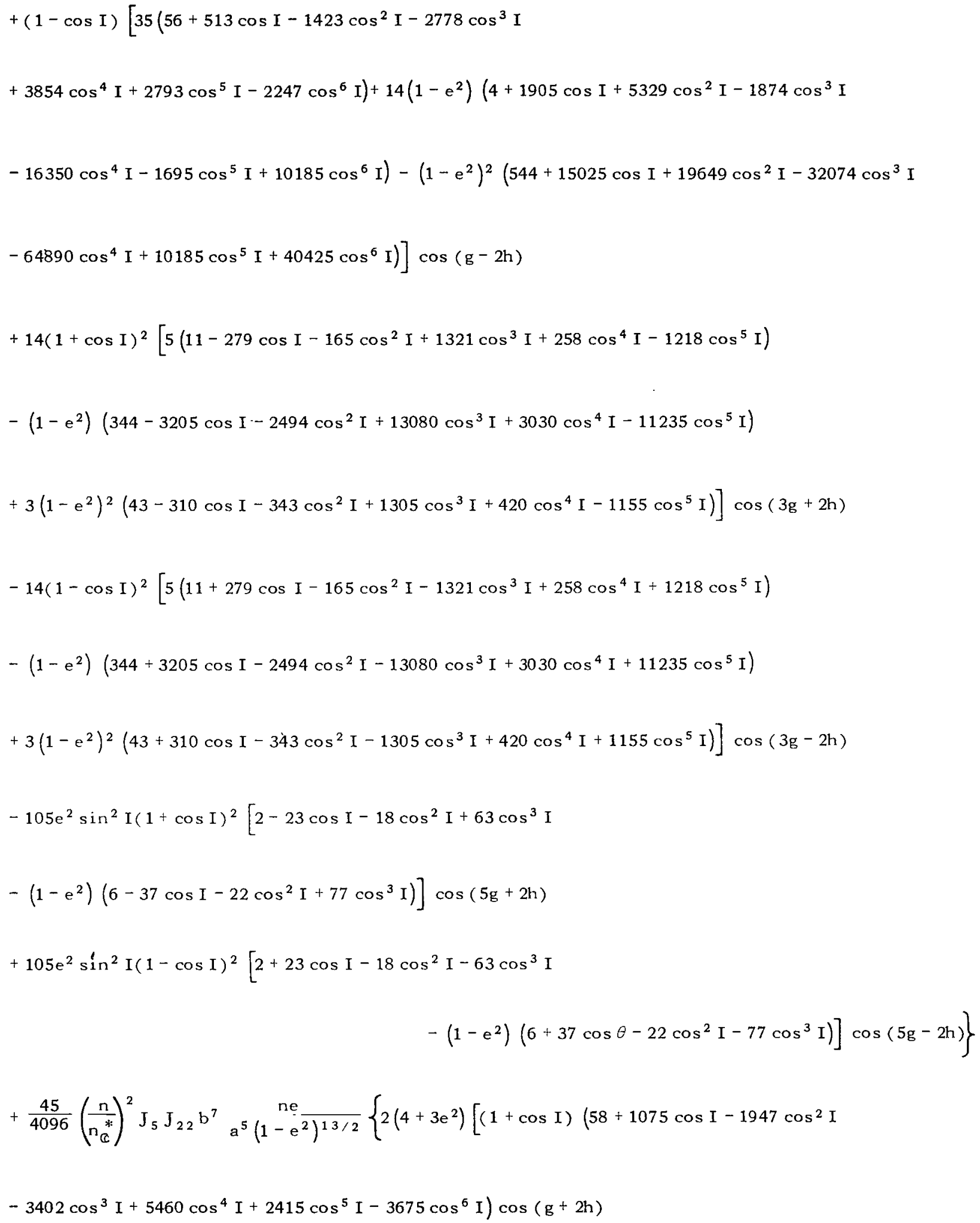




$$
\begin{aligned}
& -(1-\cos I)\left(58-1075 \cos I-1947 \cos ^{2} I+3402 \cos ^{3} I\right. \\
& \left.\left.+5460 \cos ^{4} I-2415 \cos ^{5} I-3675 \cos ^{6} I\right) \cos (g-2 h)\right] \\
& -7 e^{2} \sin ^{2} I\left[( 1 + \operatorname { c o s } I ) \left(14+209 \cos I-355 \cos ^{2} I\right.\right. \\
& \left.-345 \cos ^{3} I+525 \cos ^{4} I\right) \cos (3 g+2 h)-(1-\cos I)\left(14-209 \cos I-355 \cos ^{2} I\right. \\
& \left.\left.\left.+345 \cos ^{3} I+525 \cos ^{4} I\right) \cos (3 g-2 h)\right]\right\},
\end{aligned}
$$

$$
\begin{aligned}
& \frac{\partial S_{2}^{\prime}(\text { coupling) }}{\partial \mathrm{e}}=\frac{9}{4096 \epsilon^{2}}\left(\frac{n_{\overparen{C}}}{n}\right)^{2}\left(\frac{n_{\mathbb{C}}}{n_{\overparen{C}}^{*}}\right)^{2} \frac{n a^{2} e}{\left(1-e^{2}\right)^{3 / 2}}\left\{-96 \sin ^{2} I \cos I\left(12-17 e^{2}\right)\left(1-e^{2}\right) \sin 2 h\right. \\
& -4 \sin ^{2} I \cos I\left(2+3 e^{2}\right)\left(14-9 e^{2}\right) \sin 4 h+80(1+\cos I)^{2}(2-3 \cos I)\left(2-3 e^{2}\right)\left(1-e^{2}\right) \sin (2 g+2 h) \\
& +80(1-\cos I)^{2}(2+3 \cos I)\left(2-3 e^{2}\right)\left(1-e^{2}\right) \sin (2 g-2 h) \\
& +10 \sin ^{2} I(1+\cos I)\left[6 e^{2}\left(4-3 e^{2}\right)-\left(2-e^{2}\right)(1+5 \cos I)\right] \sin (2 g+4 h) \\
& +10 \sin ^{2} I(1-\cos I)\left[6 e^{2}\left(4-3 e^{2}\right)-\left(2-e^{2}\right)(1-5 \cos I)\right] \sin (2 g-4 h) \\
& +25(1+\cos I)^{3}\left[2 e^{2}\left(4-3 e^{2}\right)-\left(2-e^{2}\right)(1+\cos I)\right] \sin (4 g+4 h) \\
& \left.+25(1-\cos I)^{3}\left[2 e^{2}\left(4-3 e^{2}\right)-\left(2-e^{2}\right)(1-\cos I)\right] \sin (4 g-4 h)\right\} \\
& +\frac{9}{128 \epsilon}\left(\frac{\mathrm{n}_{\overparen{C}}}{\mathrm{n}_{\overparen{C}}^{*}}\right)^{2} J_{2} b^{2} \frac{n e}{\left(1-e^{2}\right)^{3}}\left[4 \sin ^{2} I \cos I\left(7+3 e^{2}\right) \sin 2 h\right. \\
& +5(1+\cos I)^{2}\left(1+2 \cos I-5 \cos ^{2} I\right)\left(1+e^{2}\right) \sin (2 g+2 h)
\end{aligned}
$$$$
\left.+5(1-\cos I)^{2}\left(1-2 \cos I-5 \cos ^{2} I\right)\left(1+e^{2}\right) \sin (2 g-2 h)\right]
$$ 


$$
\begin{aligned}
& -\frac{9}{25 \sigma \epsilon}\left(\frac{n_{C}}{n_{C}^{*}}\right)^{2} J_{22} b^{2} \begin{array}{r}
n e \sin ^{2} I \\
\left(1-e^{2}\right)^{3}
\end{array}-8 \cos I\left(7+3 e^{2}\right)(2 \sin 2 h-\sin 4 h) \\
& -20(1+\cos I)(3-5 \cos I)\left(1+e^{2}\right) \sin (2 g+2 h) \\
& -20(1-\cos I)(3+5 \cos I)\left(1+e^{2}\right) \sin (2 g-2 h) \\
& +5(1+\cos I)(1+5 \cos I)\left(1+e^{2}\right) \sin (2 g+4 h)
\end{aligned}
$$$$
\left.+5(1-\cos I)(1-5 \cos I)\left(1+e^{2}\right) \sin (2 g-4 h)\right]
$$

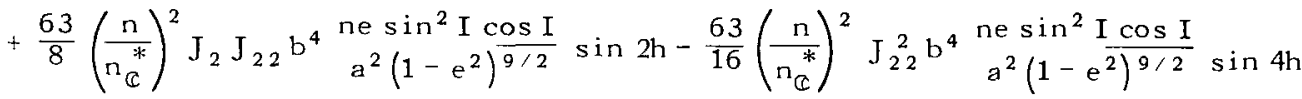$$
+10 \frac{9}{24 \epsilon}\left(\frac{n_{C}}{n_{C}^{*}}\right)^{2} J_{3} b^{3} \quad \begin{array}{cc}
n \sin \\
7\left(1-e^{2}\right)^{4}
\end{array}\left\{( 1 + \operatorname { c o s } I ) \left[150\left(1-\cos I-\cos ^{2} I-7 \cos ^{3} I\right)\right.\right.
$$$$
-\left(1-e^{2}\right)\left(161-65 \cos I-65 \cos ^{2} I-1575 \cos ^{3} I\right)+3\left(1-e^{2}\right)^{2}(9+15 \cos I
$$$$
\left.\left.+15 \cos ^{2} \mathrm{I}-175 \cos ^{3} \mathrm{I}\right)\right] \cos (\mathrm{g}+2 \mathrm{~h})+(1-\cos \mathrm{I})\left[150\left(1+\cos \mathrm{I}-\cos ^{2} \mathrm{I}+7 \cos ^{3} \mathrm{I}\right)\right.
$$$$
-\left(1-e^{2}\right)\left(161+65 \cos I-65 \cos ^{2} I+1575 \cos ^{3} I\right)
$$$$
\left.+3\left(1-e^{2}\right)^{2}\left(9-15 \cos I+15 \cos ^{2} I+175 \cos ^{3} I\right)\right] \cos (g-2 h)
$$$$
-75 e^{2}\left(1+e^{2}\right)\left[(1+\cos I)^{2}\left(1+2 \cos I-7 \cos ^{2} I\right) \cos (3 g+2 h)\right.
$$

$$
\left.\left.+(1-\cos I)^{2}\left(1-2 \cos I-7 \cos ^{2} I\right) \cos (3 g-2 h)\right]\right\}
$$

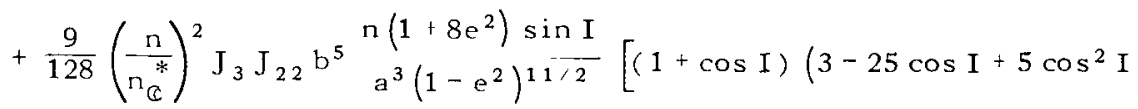

$$
\left.\left.+25 \cos ^{3} I\right) \cos (g+2 h)+(1-\cos I)\left(3+25 \cos I+5 \cos ^{2} I-25 \cos ^{3} I\right) \cos (g-2 h)\right]
$$




$$
\begin{aligned}
& +\frac{45}{2048 \epsilon}\left(\frac{n_{\mathbb{C}}}{n_{\mathbb{C}}^{*}}\right)^{2} J_{4} b^{4} \frac{n e}{a^{2}\left(1-e^{2}\right)^{5}}\left\{8 \operatorname { s i n } ^ { 2 } I \operatorname { c o s } I \left[70-15\left(1-e^{2}\right)\left(2+7 \cos ^{2} I\right)\right.\right. \\
& \left.-3\left(1-e^{2}\right)^{2}\left(1-14 \cos ^{2} I\right)\right] \sin 2 h \\
& +(1+\cos I)^{2}\left[70\left(3+4 \cos I-30 \cos ^{2} I-20 \cos ^{3} I+51 \cos ^{4} I\right)\right. \\
& -3\left(1-e^{2}\right)\left(69+124 \cos I-714 \cos ^{2} I-644 \cos ^{3} I+1365 \cos ^{4} I\right) \\
& \left.+3\left(1-e^{2}\right)^{2}\left(11+36 \cos I-126 \cos ^{2} I-196 \cos ^{3} I+315 \cos ^{4} I\right)\right] \sin (2 g+2 h) \\
& +(1-\cos I)^{2}\left[70\left(3-4 \cos I-30 \cos ^{2} I+20 \cos ^{3} I+51 \cos ^{4} I\right)\right. \\
& -3\left(1-e^{2}\right)\left(69-124 \cos I-714 \cos ^{2} I+644 \cos ^{3} I+1365 \cos ^{4} I\right) \\
& \left.+3\left(1-e^{2}\right)^{2}\left(11-36 \cos I-126 \cos ^{2} I+196 \cos ^{3} I+315 \cos ^{4} I\right)\right] \sin (2 g-2 h) \\
& -35 e^{2}\left(1+e^{2}\right) \sin ^{2} I\left[(1+\cos I)^{2}\left(1+2 \cos I-9 \cos ^{2} I\right) \sin (4 g+2 h)\right. \\
& \left.\left.+(1-\cos I)^{2}\left(1-2 \cos I-9 \cos ^{2} I\right) \sin (4 g-2 h)\right]\right\} \\
& +\frac{45}{512}\left(\frac{n}{n_{\mathbb{C}}^{*}}\right)^{2} J_{4} J_{22} b^{6} \frac{n e}{a^{4}\left(1-e^{2}\right)^{13 / 2}}\left\{4\left(28+27 e^{2}\right) \cos I\left(3-7 \cos ^{2} I\right) \sin 2 h\right. \\
& +\left(2+9 e^{2}\right)\left[(1+\cos I)\left(3-19 \cos I-7 \cos ^{2} I+35 \cos ^{3} I\right) \sin (2 g+2 h)\right. \\
& \left.\left.+(1-\cos I)\left(3+19 \cos I-7 \cos ^{2} I-35 \cos ^{3} I\right) \sin (2 g-2 h)\right]\right\} \\
& +\frac{45}{32768 \epsilon} \cdot\left(\frac{n_{C}}{n_{C}^{*}}\right)^{2} J_{5} b^{5} \frac{n \sin I}{a^{3}\left(1-e^{2}\right)^{6}}\left\{( 1 + \operatorname { c o s } I ) \left[3 5 0 \left(15+41 \cos I-262 \cos ^{2} I\right.\right.\right. \\
& \left.-346 \cos ^{3} I+519 \cos ^{4} I+321 \cos ^{5} I\right) \\
& +7\left(1-e^{2}\right)\left(3469-5925 \cos I+2734 \cos ^{2} I\right. \\
& \left.+42930 \cos ^{3} I-31755 \cos ^{4} I-37725 \cos ^{5} I\right)
\end{aligned}
$$




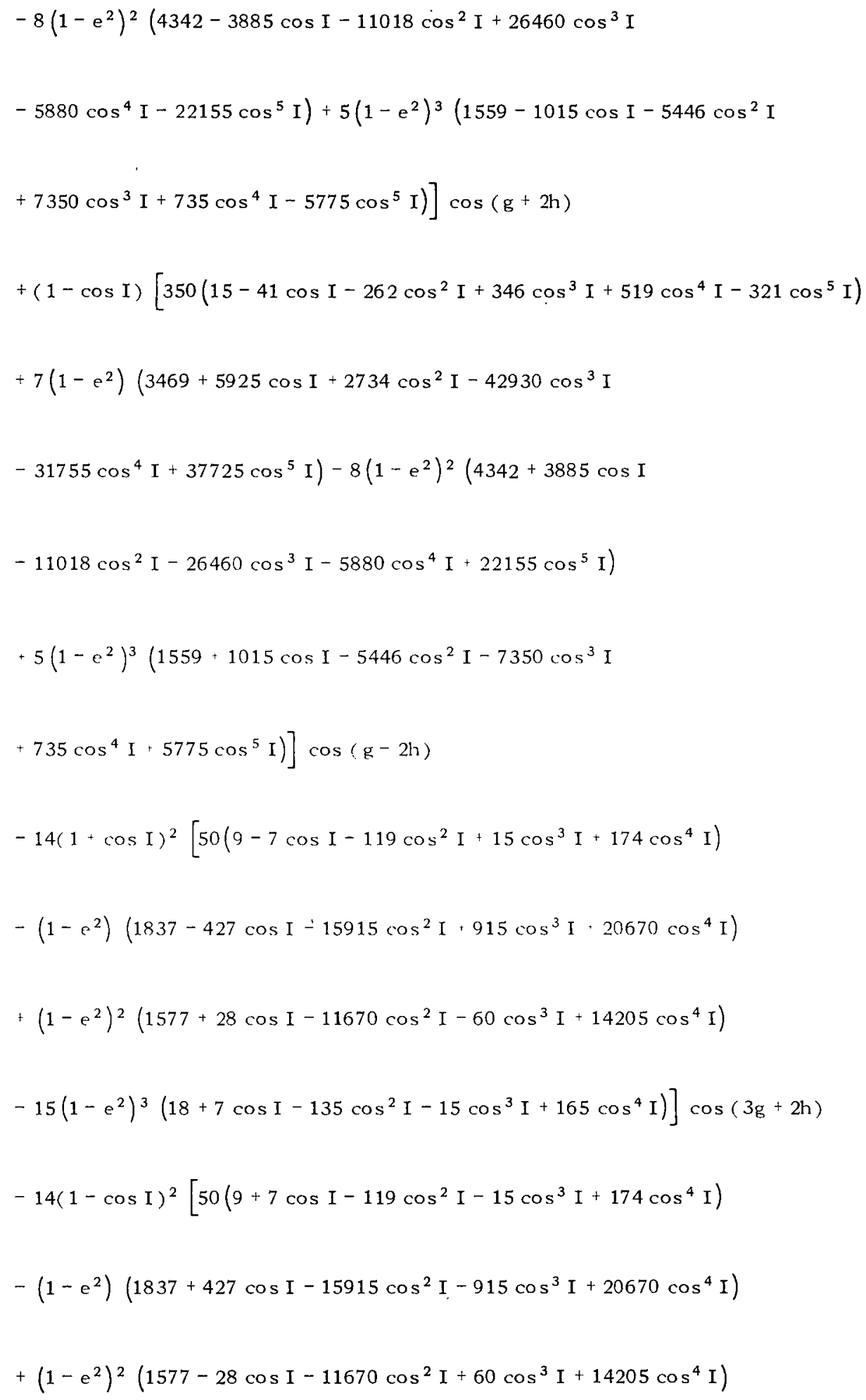




$$
\begin{aligned}
& \left.-15\left(1-e^{2}\right)^{3}\left(18-7 \cos I-135 \cos ^{2} I+15 \cos ^{3} I+165 \cos ^{4} I\right)\right] \cos (3 g-2 h) \\
& +105 e^{2} \sin ^{2} I\left[10\left(1-9 \cos ^{2} I\right)-\left(1-e^{2}\right)\left(31-151 \cos ^{2} I\right)\right. \\
& \left.\left.+5\left(1-e^{2}\right)^{2}\left(3-11 \cos ^{2} I\right)\right]\left[(1+\cos I)^{2} \cos (5 g+2 h)+(1-\cos I)^{2} \cos (5 g-2 h)\right]\right\} \\
& +\frac{45}{4096}\left(\frac{n}{n_{C}^{*}}\right)^{2} J_{5} J_{22} b^{7} \frac{n \sin I}{a^{5}\left(1-e^{2}\right)^{15 / 2}}\left\{2 ( 4 + 5 7 e ^ { 2 } + 3 0 e ^ { 4 } ) \left(29-126 \cos ^{2} I\right.\right. \\
& \left.+105 \cos ^{4} I\right)[(1+\cos I)(3-5 \cos I) \cos (g+2 h)+(1-\cos I)(3+5 \cos I) \cos (g-2 h)] \\
& -7 e^{2}\left(3+10 e^{2}\right) \sin ^{2} I\left(7-15 \cos ^{2} I\right)[(1+\cos I)(3-5 \cos I) \cos (3 g+2 h) \\
& +(1-\cos I)(3+5 \cos I) \cos (3 g-2 h)]\} \\
& {\underline{\partial S_{2}^{\prime}}}_{\partial g}^{(\text {coupling })}=\frac{45}{1024 \epsilon^{2}}\left(\frac{n_{\mathbb{C}}}{n}\right)^{2}\left(\frac{n_{C}}{n_{C}^{*}}\right)^{2} \frac{n a^{2}}{\left(1-e^{2}\right)^{1 / 2}}\left[8 C_{3} \cos (2 g+2 h)+8 C_{4} \cos (2 g-2 h)\right. \\
& \left.+C_{5} \cos (2 g+4 h)+C_{6} \cos (2 g-4 h)+5 C_{7} \cos (4 g+4 h)+5 C_{8} \cos (4 g-4 h)\right] \\
& +\frac{45}{128 \epsilon}\left(\frac{n_{C}}{n_{C}^{*}}\right)^{2} J_{2} b^{2} \frac{n}{\left(1-e^{2}\right)^{2}}\left[C_{10} \cos (2 g+2 h)+C_{11} \cos (2 g-2 h)\right] \\
& -\frac{45}{256 \epsilon}\left(\frac{n_{\mathbb{C}}}{n_{\mathbb{C}}^{*}}\right)^{2} J_{22} b^{2} \frac{n \sin ^{2} I}{\left(1-e^{2}\right)^{2}}\left[-4 C_{13} \cos (2 g+2 h)-4 C_{14} \cos (2 g-2 h)\right. \\
& \left.+C_{15} \cos (2 g+4 h)+C_{16} \cos (2 g-4 h)\right] \\
& -\frac{9}{1024 \epsilon}\left(\frac{n_{C}}{n_{C}^{*}}\right)^{2} J_{3} b^{3} \frac{n e \sin I}{a\left(1-e^{2}\right)^{3}}\left[C_{17} \sin (g+2 h)+C_{18} \sin (g-2 h)\right. \\
& \left.-75 C_{19} \sin (3 g+2 h)-75 C_{20} \sin (3 g-2 h)\right] \\
& -\frac{9}{128}\left(\frac{n}{n_{đ}^{*}}\right)^{2} J_{3} J_{22} b^{5} \frac{n e \sin I}{a^{3}\left(1-e^{2}\right)^{9 / 2}}\left[C_{21} \sin (g+2 h)+C_{22} \sin (g-2 h)\right]
\end{aligned}
$$




$$
\begin{aligned}
& +\frac{45}{4096 \epsilon}\left(\frac{n_{\bigodot}}{n_{\bigodot}^{*}}\right)^{2} J_{4} b^{4} \frac{n}{a^{2}\left(1-e^{2}\right)^{4}}\left[C_{24} \cos (2 g+2 h)+C_{25} \cos (2 g-2 h)\right. \\
& \left.-70 C_{26} \cos (4 g+2 h)-70 C_{27} \cos (4 g-2 h)\right] \\
& +\frac{45}{256}\left(\frac{n}{n_{C}^{*}}\right)^{2} J_{4} J_{22} b^{6} \frac{n \sin ^{2} I}{a^{4}\left(1-e^{2}\right)^{1 / / 2}}\left[C_{29} \cos (2 g+2 h)+C_{30} \cos (2 g-2 h)\right] \\
& -\frac{45}{32768 \epsilon}\left(\frac{n_{C}}{n_{C}}\right)^{2} J_{5} b^{5} \frac{n e \sin I}{a^{3}\left(1-e^{2}\right)^{5}}\left[C_{31} \sin (g+2 h)+C_{32} \sin (g-2 h)-42 C_{33} \sin (3 g+2 h)\right. \\
& \left.-42 C_{34} \sin (3 g-2 h)+525 C_{35} \sin (5 g+2 h)+525 C_{36} \sin (5 g-2 h)\right]
\end{aligned}
$$

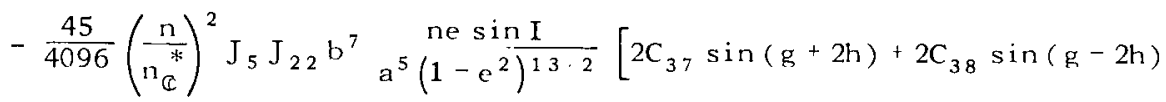

$$
\begin{aligned}
& \left.-21 C_{39} \sin (3 g+2 h)-21 C_{40} \sin (3 g-2 h)\right] \text {, }
\end{aligned}
$$

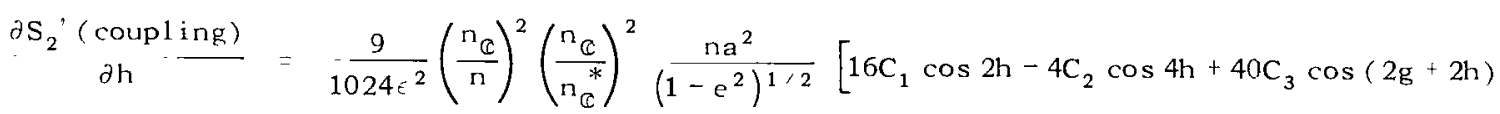

$$
\begin{aligned}
& -40 C_{4} \cos (2 g-2 h)+10 C_{5} \cos (2 g+4 h)-10 C_{6} \cos (2 g-4 h) \\
& \left.+25 C_{7} \cos (4 g+4 h)-25 C_{8} \cos (4 g-4 h)\right] \\
& +\frac{9}{128 \epsilon}\left(\frac{n_{C}}{n_{C}^{*}}\right)^{2} J_{2} b^{2} \frac{n}{\left(1-e^{2}\right)^{2}}\left[4 C_{9} \cos 2 h+5 C_{10} \cos (2 g+2 h)-5 C_{11} \cos (2 g-2 h)\right]
\end{aligned}
$$

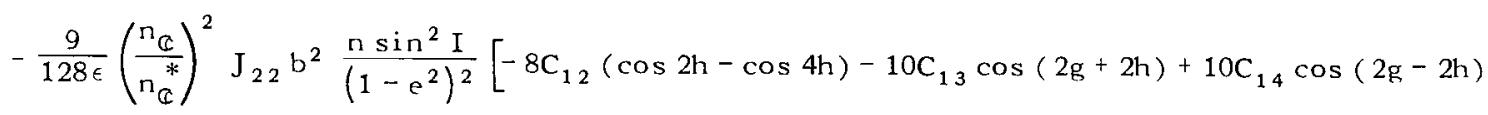

$$
\begin{aligned}
& \left.+5 C_{15} \cos (2 g+4 h)-5 C_{16} \cos (2 g-4 h)\right]
\end{aligned}
$$




$$
\begin{aligned}
& +\frac{9}{4}\left(\frac{n}{n_{C}^{*}}\right)^{2} J_{2} J_{22} b^{4} \frac{n \sin ^{2} I}{a^{2}\left(1-e^{2}\right)^{7 / 2}} \cos I \frac{9}{4}\left(\frac{n}{n_{C}^{*}}\right)^{2} J_{22}^{2} b^{4} \frac{n \sin ^{2} I}{a^{2}\left(1-e^{2}\right)^{-\frac{1}{7 / 2}}} \cos 4 h \\
& -\frac{9}{512 \epsilon}\left(\frac{n_{\overparen{C}}}{n_{\overparen{C}}^{*}}\right)^{2} J_{3} b^{3} \frac{n e \sin I}{a\left(1-e^{2}\right)^{3}}\left[C_{17} \sin (g+2 h)-C_{18} \sin (g-2 h)-25 C_{19} \sin (3 g+2 h)+25 C_{20} \sin (3 g-2 h)\right] \\
& -\frac{9}{64}\left(\frac{n}{n_{C}^{*}}\right)^{2} J_{3} J_{22} b^{5} \frac{n e \sin I}{a^{3}\left(1-e^{2}\right)^{9 / 2}}\left[C_{21} \sin (g+2 h)-C_{22} \sin (g-2 h)\right] \\
& +\frac{45}{4096 \epsilon}\left(\frac{n_{\overparen{C}}}{n_{\mathbb{C}}^{*}}\right)^{2} J_{4} b^{4} \frac{n}{a^{2}\left(1-e^{2}\right)^{4}}\left[8 C_{23} \cos 2 h+C_{24} \cos (2 g+2 h)-C_{25} \cos (2 g-2 h)\right. \\
& \left.-35 C_{26} \cos (4 g+2 h)+35 C_{27} \cos (4 g-2 h)\right] \\
& +\frac{45}{256}\left(\frac{n}{n_{C}^{*}}\right)^{2} J_{4} J_{22} b^{6} \frac{n \sin ^{2}}{a^{4}\left(1-e^{2}\right)^{112}}\left[4 C_{28} \cos 2 h+C_{29} \cos (2 g+2 h)-C_{30} \cos (2 g-2 h)\right] \\
& -\frac{45}{16384 \epsilon}\left(\frac{n_{\mathbb{C}}}{n_{\mathbb{C}}^{*}}\right)^{2} J_{5} b^{5} \frac{n e \sin I}{a^{3}\left(1-\frac{e^{2}}{)^{5}}\right.}\left[C_{31} \sin (g+2 h)-C_{32} \sin (g-2 h)-14 C_{33} \sin (3 g+2 h)\right. \\
& \left.+14 C_{34} \sin (3 g-2 h)+105 C_{35} \sin (5 g+2 h)-105 C_{36} \sin (5 g-2 h)\right] \\
& -\frac{45}{2048}\left(\frac{n}{n_{\mathbb{C}}^{*}}\right)^{2} J_{5} J_{22} b^{7} \frac{n e}{a^{5}\left(1-c^{2}\right)^{132}}\left[2 C_{37} \sin (g+2 h)-2 C_{38} \sin (g-2 h)\right. \\
& \left.-7 \mathrm{C}_{39} \sin (3 g+2 h)+7 \mathrm{C}_{40} \sin (3 g-2 h)\right] \\
& \frac{\partial S_{2}^{\prime}(e a r t h)}{\partial a}=\frac{5}{512 \epsilon}\left(\frac{n_{\mathbb{C}}}{n_{\mathbb{C}}^{*}}\right) \frac{n_{\mathbb{C}} a^{2} e}{a_{\mathbb{C}}}\left[-9(1+\cos I)\left(1+10 \cos I-15 \cos ^{2} I\right)\left(4+3 e^{2}\right) \sin (g+h)\right. \\
& +9(1-\cos I)\left(1-10 \cos I-15 \cos ^{2} I\right)\left(4+3 e^{2}\right) \sin (g-h) \\
& +15 \sin ^{2} I(1+\cos I)\left(4+3 e^{2}\right) \sin (g+3 h) \\
& -15 \sin ^{2} I(1-\cos I)\left(4+3 e^{2}\right) \sin (g-3 h)
\end{aligned}
$$




$$
\begin{aligned}
& +315 \sin ^{2} I(1+\cos I) e^{2} \sin (3 g+h) \\
& -315 \sin ^{2} I(1-\cos I) e^{2} \sin (3 g-h) \\
& \left.\quad+35(1+\cos I)^{3} e^{2} \sin (3 g+3 h)-35(1-\cos I)^{3} e^{2} \sin (3 g-3 h)\right]
\end{aligned}
$$

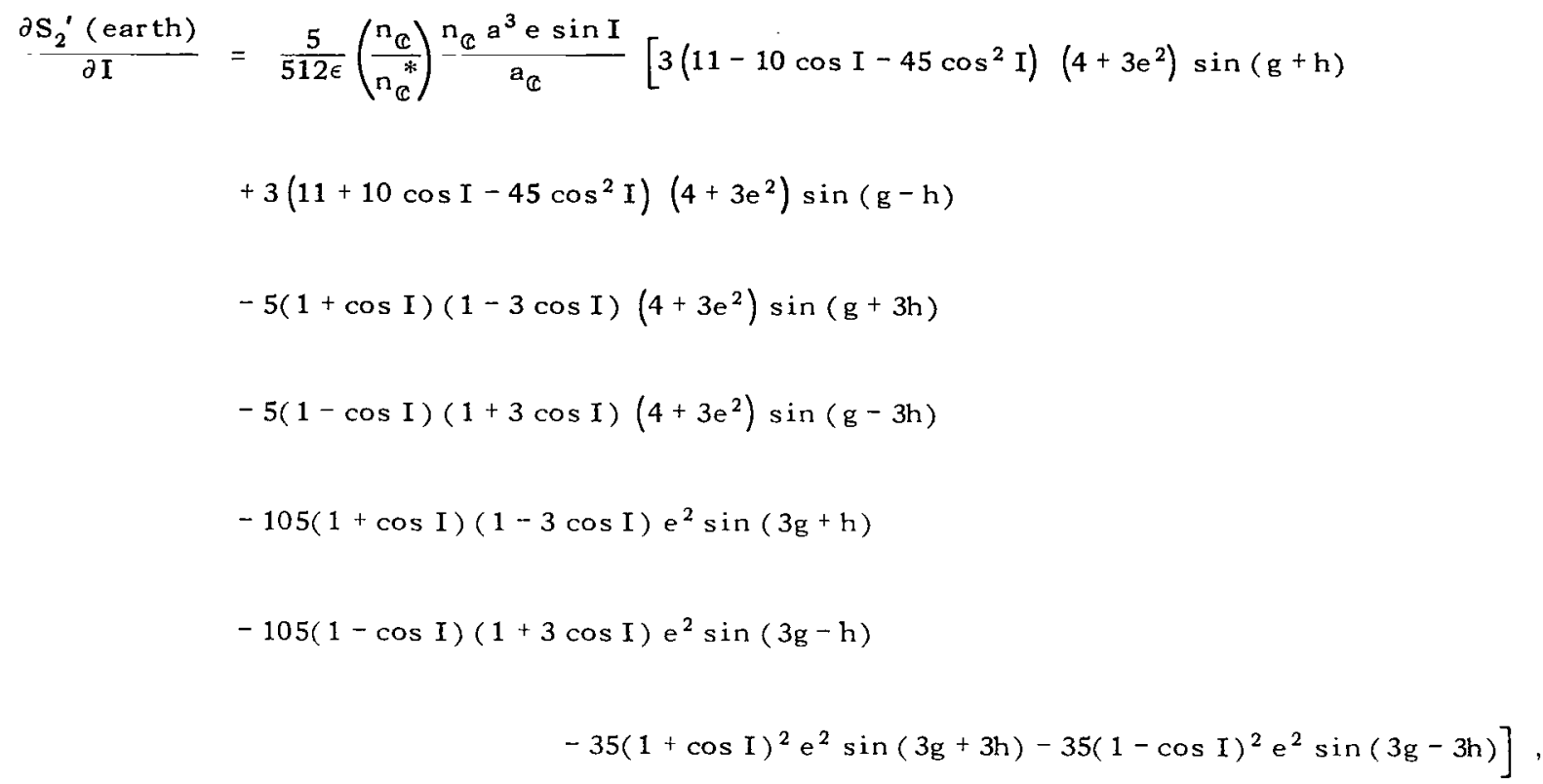




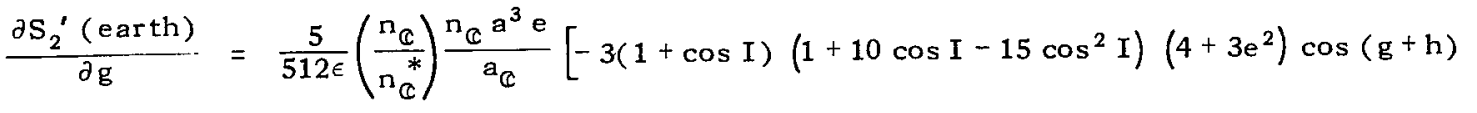

$$
\begin{aligned}
& +3(1-\cos I)\left(1-10 \cos I-15 \cos ^{2} I\right)\left(4+3 e^{2}\right) \cos (g-h) \\
& +5 \sin ^{2} I(1+\cos I)\left(4+3 e^{2}\right) \cos (g+3 h) \\
& -5 \sin ^{2} I(1-\cos I)\left(4+3 e^{2}\right) \cos (g-3 h) \\
& +315 \sin ^{2} I(1+\cos I) e^{2} \cos (3 g+h) \\
& -315 \sin ^{2} I(1-\cos I) e^{2} \cos (3 g-h) \\
& \left.+35(1+\cos I)^{3} e^{2} \cos (3 g+3 h)-35(1-\cos I)^{3} e^{2} \cos (3 g-3 h)\right] \\
& \frac{\partial S_{2}^{\prime}(\text { earth })}{\partial h}=\frac{5}{512 \epsilon}\left(\frac{n_{C}}{n_{C}^{*}}\right) \frac{n_{\mathbb{C}} a^{3} e}{a_{\mathbb{C}}}\left[-3(1+\cos I)\left(1+10 \cos I-15 \cos ^{2} I\right)\left(4+3 e^{2}\right) \cos (g+h)\right. \\
& -3(1-\cos I)\left(1-10 \cos I-15 \cos ^{2} I\right)\left(4+3 e^{2}\right) \cos (g-h) \\
& +15 \sin ^{2} I(1+\cos I)\left(4+3 e^{2}\right) \cos (g+3 h) \\
& +15 \sin ^{2} I(1-\cos I)\left(4+3 e^{2}\right) \cos (g-3 h) \\
& +105 \sin ^{2} I(1+\cos I) e^{2} \cos (3 g+h) \\
& +105 \sin ^{2} I(1-\cos I) e^{2} \cos (3 g-h) \\
& \left.+35(1+\cos I)^{3} e^{2} \cos (3 g+3 h)+35(1-\cos I)^{3} e^{2} \cos (3 g-3 h)\right]
\end{aligned}
$$

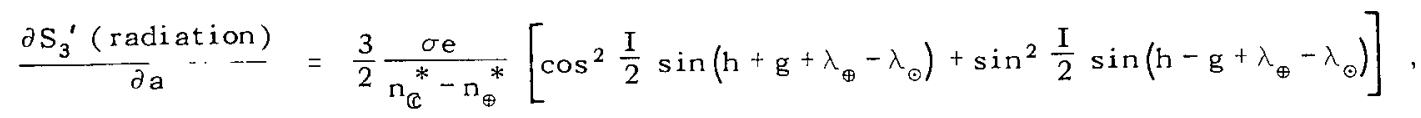

$$
\begin{aligned}
& \frac{\partial \mathrm{S}_{3}^{\prime}(\text { radiation })}{\partial \mathrm{I}}-\cdots \frac{3}{4} \frac{\sigma \mathrm{ae} \sin \mathrm{I}}{\mathrm{n}_{\odot}^{*}-\mathrm{n}_{\oplus}^{*}}\left[-\sin \left(\mathrm{h}+\mathrm{g}+\lambda_{\oplus}-\lambda_{\odot}\right)+\sin \left(\mathrm{h}-\mathrm{g}+\lambda_{\oplus}-\lambda_{\odot}\right)\right]
\end{aligned}
$$




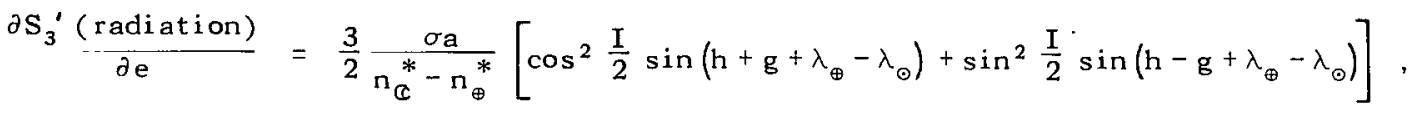

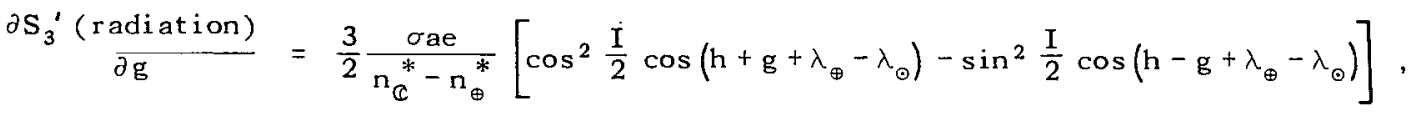

$$
\begin{aligned}
& \frac{\partial \mathrm{S}_{3}{ }^{\prime} \text { (radiation) }}{\partial \bar{h}}=\frac{3}{2} \frac{\sigma a \mathrm{e}}{\mathrm{n}_{\mathbb{C}}^{*}-\mathrm{n}_{\oplus}^{*}}\left[\cos ^{2} \frac{\mathrm{I}}{2} \cos \left(\mathrm{h}+\mathrm{g}+\lambda_{\oplus}-\lambda_{\odot}\right)+\sin ^{2} \frac{\mathrm{I}}{2} \cos \left(\mathrm{h}-\mathrm{g}+\lambda_{\oplus}-\lambda_{\odot}\right)\right], \\
& \frac{\partial S_{3}{ }^{\prime}(\text { sun })}{\partial a}=\frac{3}{32} \frac{n_{\oplus}^{2} a}{n_{\overparen{C}}^{*}-n_{\oplus}^{*}}\left[2\left(2+3 e^{2}\right) \sin ^{2} I \sin 2\left(h+\lambda_{\oplus}-\lambda_{\odot}\right)\right. \\
& \left.+5 \mathrm{e}^{2}(1+\cos \mathrm{I})^{2} \sin 2\left(\mathrm{~h}+\mathrm{g}+\lambda_{\oplus}-\lambda_{\odot}\right)+5 \mathrm{e}^{2}(1-\cos \mathrm{I})^{2} \sin 2\left(\mathrm{~h}-\mathrm{g}+\lambda_{\oplus}-\lambda_{\odot}\right)\right]
\end{aligned}
$$

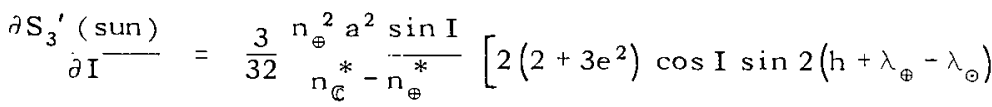

$$
\begin{aligned}
& \left.-5 \mathrm{e}^{2}(1+\cos \mathrm{I}) \sin 2\left(\mathrm{~h}+\mathrm{g}+\lambda_{\oplus}-\lambda_{\odot}\right)+5 \mathrm{e}^{2}(1-\cos \mathrm{I}) \sin 2\left(\mathrm{~h}-\mathrm{g}+\lambda_{\oplus}-\lambda_{\odot}\right)\right]
\end{aligned}
$$

$$
\begin{aligned}
& \partial S_{3}{ }^{\prime} \frac{(\operatorname{sun})}{\partial \bar{e}}=\frac{3}{32} \frac{n_{\oplus}^{2} a^{2} e}{n_{\mathscr{C}}^{*}-n_{\oplus}^{*}}\left[6 \sin ^{2} I \sin 2\left(h+\lambda_{\oplus}-\lambda_{\odot}\right)\right. \\
& \left.+5(1+\cos I)^{2} \sin 2\left(h+g+\lambda_{\oplus}-\lambda_{\Theta}\right)+5(1-\cos I)^{2} \sin 2\left(h-g+\lambda_{\oplus}-\lambda_{\Theta}\right)\right],
\end{aligned}
$$

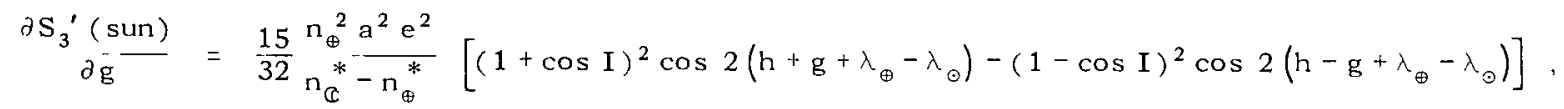

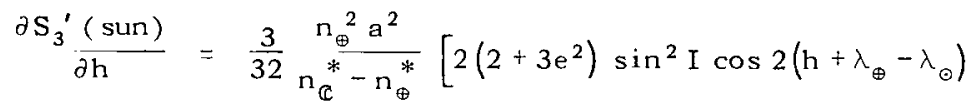

$$
\begin{aligned}
& \left.+5 e^{2}(1+\cos \mathrm{I})^{2} \cos 2\left(\mathrm{~h}+\mathrm{g}+\lambda_{\oplus}-\lambda_{\odot}\right)+5 \mathrm{e}^{2}(1-\cos \mathrm{I})^{2} \cos 2\left(\mathrm{~h}-\mathrm{g}+\lambda_{\oplus}-\lambda_{\Theta}\right)\right]
\end{aligned}
$$

$$
\frac{\partial S_{3}^{\prime}\left(e_{\mathbb{C}}\right)}{\partial a}=\frac{3}{32 \epsilon} n_{\mathbb{C}} e_{\mathbb{C}} a\left[-2\left(2+3 e^{2}\right)\left(1-3 \cos ^{2} I\right)+30 e^{2} \sin ^{2} I \cos 2 g\right.
$$




$$
\begin{aligned}
+6\left(2+3 e^{2}\right) \sin ^{2} I \cos 2 h+15 e^{2}(1+\cos I)^{2} \cos (2 g+2 h) \\
\\
\left.+15 e^{2}(1-\cos I)^{2} \cos (2 g-2 h)\right] \sin l_{\oplus}
\end{aligned}
$$

$$
\begin{aligned}
& \frac{\partial S_{3}{ }^{\prime}\left(e_{C}\right)}{\partial I}=\frac{9}{32 \epsilon} n_{\mathbb{C}} e_{\mathbb{C}} a^{2} \sin I\left[-2\left(2+3 e^{2}\right) \cos I+10 e^{2} \cos I \cos 2 g\right. \\
& +2\left(2+3 e^{2}\right) \cos I \cos 2 h-5 e^{2}(1+\cos I) \cos (2 g+2 h) \\
& \left.+5 e^{2}(1-\cos I) \cos (2 g-2 h)\right] \sin l_{\oplus}, \\
& \left.+5(1+\cos I)^{2} \cos (2 g+2 h)+5(1-\cos I)^{2} \cos (2 g-2 h)\right] \sin l_{\oplus},
\end{aligned}
$$

$$
\begin{aligned}
\frac{\partial S_{3}{ }^{\prime}\left(e_{C}\right)}{\partial g}=-\frac{45}{32 \epsilon} n_{\mathbb{C}} e_{\mathbb{C}} a^{2} e^{2}\left[2 \sin ^{2} I \sin 2 g+(1+\cos I)^{2} \sin (2 g+2 h)\right. \\
\left.+(1-\cos \mathrm{I})^{2} \sin (2 g-2 h)\right] \sin l_{\oplus}
\end{aligned}
$$

$\frac{\partial S_{3}^{\prime}\left(e_{\mathbb{C}}\right)}{\partial h}=-\frac{9}{32 \epsilon} n_{\mathbb{C}} e_{\mathbb{C}} a^{2}\left[2\left(2+3 e^{2}\right) \sin ^{2} I \sin 2 h+5 e^{2}(1+\cos I)^{2} \sin (2 g+2 h)\right.$

$$
\left.-5 \mathrm{e}^{2}(1-\cos \mathrm{I})^{2} \sin (2 \mathrm{~g}-2 \mathrm{~h})\right] \sin l_{\oplus}
$$

$$
\begin{aligned}
& \frac{\partial S_{3}^{\prime}\left(i_{\mathbb{C}}\right)}{\partial a}=-\frac{3}{4 \epsilon} \frac{n_{\mathbb{C}}^{2} a \sin I \sin i_{\mathbb{C}}}{\bar{n}_{\mathbb{C}}+\bar{N}_{\omega C}}\left[-2\left(2+3 e^{2}\right) \cos I \sinh \right. \\
& \left.+5 e^{2}(1+\cos I) \sin (2 g+h)+5 e^{2}(1-\cos I) \sin (2 g-h)\right] \cos v_{\oplus},
\end{aligned}
$$

$$
\begin{aligned}
\frac{\partial S_{3}{ }^{\prime}\left(i_{\mathbb{C}}\right)}{\partial \mathrm{I}} & =-\frac{3}{8 \epsilon} \frac{\mathrm{n}_{\overparen{C}}^{2} \mathrm{a}^{2} \sin \mathrm{i}_{\mathbb{C}}}{\mathrm{n}_{\mathbb{C}}+\mathrm{N}_{\omega \mathbb{C}}}\left[2\left(2+3 \mathrm{e}^{2}\right)\left(1-2 \cos ^{2} \mathrm{I}\right) \sinh \right. \\
& \left.-5 \mathrm{e}^{2}(1+\cos \mathrm{I})(1-2 \cos \mathrm{I}) \sin (2 \mathrm{~g}+\mathrm{h})+5 \mathrm{e}^{2}(1-\cos \mathrm{I})(1+2 \cos \mathrm{I}) \sin (2 \mathrm{~g}-\mathrm{h})\right] \cos \mathrm{v}_{\oplus},
\end{aligned}
$$




$$
\begin{aligned}
& \partial \mathrm{S}_{3}^{\prime} \frac{\left(\mathrm{i}_{\mathbb{C}}\right)}{\partial \mathrm{e}}=-\frac{3}{4 \epsilon} \frac{\mathrm{n}_{\mathbb{C}}^{2} \mathrm{a}^{2} \mathrm{e} \sin \mathrm{I} \sin \mathrm{i}_{\mathbb{C}}}{\mathrm{n}_{\mathbb{C}}+\mathrm{N}_{\omega \mathbb{C}}}[-6 \cos \mathrm{I} \sin \mathrm{h}+5(1+\cos \mathrm{I}) \sin (2 \mathrm{~g}+\mathrm{h}) \\
& +5(1-\cos I) \sin (2 g-h)] \cos v_{\oplus}
\end{aligned}
$$

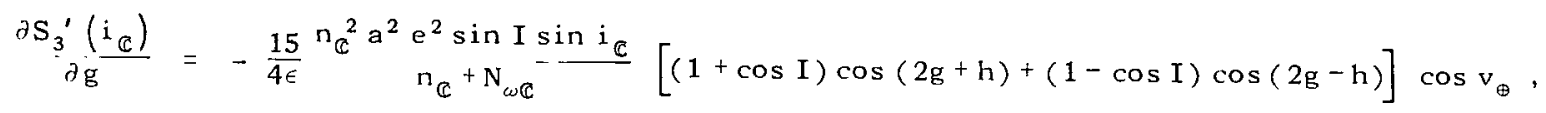

$$
\begin{aligned}
& \partial \mathrm{S}_{3}^{\prime} \underset{\partial \mathrm{h}}{\left(\mathrm{i}_{\mathbb{C}}\right)}=-\frac{3}{8 \epsilon} \stackrel{\mathrm{n}_{\mathbb{C}}^{2} \mathrm{a}^{2} \sin \mathrm{I} \sin \mathrm{i}_{\mathbb{C}}}{\mathrm{n}_{\mathbb{C}}+\mathrm{N}_{\omega \mathbb{C}}}\left[-2\left(2+3 \mathrm{e}^{2}\right) \cos \mathrm{I} \cos \mathrm{h}\right. \\
& \left.+5 e^{2}(1+\cos I) \cos (2 g+h)-5 e^{2}(1-\cos I) \cos (2 g-h)\right] \cos v_{\oplus}, \\
& \operatorname{aS}_{3}^{\prime}(4)-0
\end{aligned}
$$

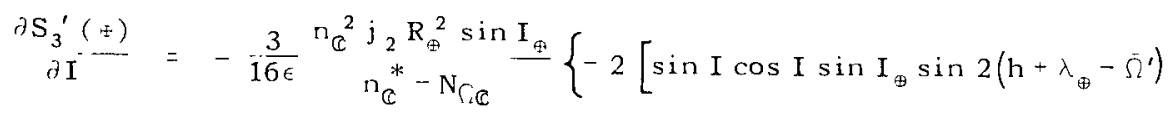

$$
\begin{aligned}
& \left.-4\left(1-2 \cos ^{2} I\right) \cos I_{\oplus} \sin \left(h+\lambda_{\oplus}-\Omega^{\prime}\right)\right] \\
& +y^{2}\left(1+2 \sqrt{1-\overline{e^{2}}}\right)\left[\sin I(1+\cos I) \sin I_{\oplus} \sin 2\left(h+g+1-\overline{\Omega^{\prime}}\right)\right. \\
& -\sin I(1-\cos I) \sin I_{f} \sin 2\left(h-g+\lambda_{f}-\bar{S}^{\prime}\right) \\
& -4(1+\cos I)(1-2 \cos I) \cos I_{\oplus} \sin \left(h+2 g+\lambda_{\oplus}-\bar{\Omega}^{\prime}\right) \\
& \left.\left.-4(1-\cos I)(1+2 \cos I) \cos I_{\oplus} \sin \left(h-2 g+\lambda_{\oplus}-\bar{\Omega}^{\prime}\right)\right]\right\} \\
& \frac{\partial S_{3}^{\prime}(\oplus)}{\partial e}=-\frac{3}{16 \epsilon}{ }^{n_{C}^{2} j_{2} R_{\oplus}^{2} \beta^{2} \sin I_{\oplus}\left(2+\sqrt{1-e^{2}}\right)}-\left[-(1+\cos I)^{2} \sin I_{\oplus} \sin 2\left(h+g+\lambda_{\oplus}-\bar{\Omega}^{\prime}\right)\right. \\
& -(1-\cos I)^{2} \sin I_{\oplus} \sin 2\left(h-g+\lambda_{\oplus}-\bar{\Omega}^{\prime}\right)
\end{aligned}
$$


$+8 \sin I(1+\cos I) \cos I_{\oplus} \sin \left(h+2 g+\lambda_{\oplus}-\bar{\Omega}^{\prime}\right)$

$\left.-8 \sin I(1-\cos I) \cos I_{\oplus} \sin \left(h-2 g+\lambda_{\oplus}-\bar{\Omega}^{\prime}\right)\right]$

$$
\begin{aligned}
\frac{\partial S_{3}^{\prime}(\oplus)}{\partial g}= & -\frac{3}{16 \epsilon} \frac{n_{\overparen{C}}^{2} j_{2} R_{\oplus}^{2} \beta^{2} \sin I_{\oplus}\left(1+2 \sqrt{1-e^{2}}\right)}{e\left(n_{\mathbb{C}}^{*}-N_{\Omega \complement}\right)}\left[-(1+\cos I)^{2} \sin I_{\oplus} \cos 2\left(h+g+\lambda_{\oplus}-\bar{\Omega}^{\prime}\right)\right. \\
& +(1-\cos I)^{2} \sin I_{\oplus} \cos 2\left(h-g+\lambda_{\oplus}-\bar{\Omega}^{\prime}\right) \\
& +8 \sin I(1+\cos I) \cos I_{\oplus} \cos \left(h+2 g+\lambda_{\oplus}-\bar{\Omega}^{\prime}\right) \\
& \left.+8 \sin I(1-\cos I) \cos I_{\oplus} \cos \left(h-2 g+\lambda_{\oplus}-\bar{\Omega}^{\prime}\right)\right]
\end{aligned}
$$

$$
\begin{aligned}
& \frac{\partial S_{3}{ }^{\prime}(\oplus)}{\partial h}=-\frac{3}{16 \epsilon} \frac{n_{\varrho}{ }^{2} j_{2} R_{\oplus}{ }^{2}}{n_{\bigodot}{ }^{*}-N_{\Omega \complement}} \frac{\sin I_{\oplus}}{n_{\Omega \complement}}\left\{-2 \sin I\left[\sin I \sin I_{\oplus} \cos 2\left(h+\lambda_{\oplus}-\bar{\Omega}^{\prime}\right)\right.\right. \\
& \left.+4 \cos I \cos I_{\oplus} \cos \left(h+\lambda_{\oplus}-\bar{\Omega}^{\prime}\right)\right] \\
& +\beta^{2}\left(1+2 \sqrt{1-e^{2}}\right)\left[-(1+\cos I)^{2} \sin I_{\oplus} \cos 2\left(h+g+\lambda_{\oplus}-\bar{\Omega}^{\prime}\right)\right. \\
& -(1-\cos \mathrm{I})^{2} \sin \mathrm{I}_{\oplus} \cos 2\left(\mathrm{~h}-\mathrm{g}+\lambda_{\oplus}-\bar{\Omega}^{\prime}\right) \\
& +4 \sin I(1+\cos I) \cos I_{\oplus} \cos \left(h+2 g+\lambda_{f}-\bar{\Omega}^{\prime}\right) \\
& \left.\left.-4 \sin I(1-\cos I) \cos I_{\oplus} \cos \left(h-2 g+\lambda_{\Theta}-\bar{\Omega}^{\prime}\right)\right]\right\} \text {. }
\end{aligned}
$$

$$
\begin{aligned}
& \frac{\partial S_{3}^{\prime}(1 \mathrm{ib} .)}{\partial \mathrm{a}}=0, \\
& \frac{\partial \mathrm{S}_{3}^{\prime}(1 \mathrm{ib} .)}{\partial I}=-2 a \mathrm{G} \sin \mathrm{I} \sin l_{\odot}, \\
& \frac{\partial \mathrm{S}_{3}^{\prime}(1 \mathrm{ib} .)}{\partial \mathrm{e}}=0, \\
& \frac{\partial \mathrm{S}_{3}^{\prime}(1 \mathrm{ib} .)}{\partial \mathrm{g}}=0,
\end{aligned}
$$


and

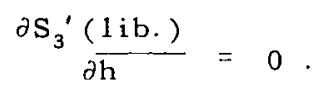

\section{SECULAR PERTURBATIONS AND PERTURBATIONS DEPENDING STRICTLY ON $\mathbf{g}$ "}

At this stage of the problem, the remaining part of the Hamiltonian is

$$
\mathfrak{F}^{\prime \prime}=\mathcal{F}_{0}^{\prime \prime}+\mathfrak{F}_{1}^{\prime \prime}+\mathfrak{F}_{2}^{\prime \prime}+\mathfrak{F}_{3}^{\prime \prime},
$$

where

$$
\begin{aligned}
& \exists_{0}^{\prime \prime}=\frac{\mu^{2}}{2 L^{\prime 2}}-\mathrm{T}=\text { const. }, \\
& \hat{H}_{1}^{\prime \prime}=\mathrm{n}_{C}^{*} \mathrm{H}=\text { const., } \\
& { }_{2}=\mathrm{F}_{2}^{\prime \prime}\left(-, \mathrm{g}^{\prime \prime},-, \mathrm{L}^{\prime}, \mathrm{G}^{\prime \prime}, \mathrm{H}^{\prime \prime}\right),
\end{aligned}
$$

and

$$
7_{3}^{\prime \prime}=F_{3}^{\prime \prime}\left(-, g^{\prime \prime},-, L^{\prime}, G^{\prime \prime}, H\right)
$$

and $\mathrm{L}^{\prime}, \mathrm{T}$, and $\mathrm{H}^{\prime \prime}$ are constants in time. $\mathrm{T}$ is effectively a third-order quantity; but in the manipulation of von Zeipel's method its contributions are of the zero order, for it is a variable itself, independent of all the others.

It is easy to see that the variable part of the Hamiltonian " $i$, which will generate the equations of motion, is factored by small parameters. Therefore, a method of successive approximations such as von Zeipel's cannot be applied. The system of differential equations produced by " ${ }^{\prime \prime}$ must be integrated directly or by using some other kind of approximation.

In a previous version of this theory (Reference 5), the system of differential equations

$$
\begin{aligned}
& \dot{\mathrm{G}}^{\prime \prime}=\frac{\partial \mathrm{F}^{\prime \prime}}{\partial \mathrm{g}^{\prime \prime}}, \\
& \dot{\mathrm{L}}^{\prime \prime}=-\frac{\partial \mathrm{F}^{\prime \prime}}{\partial \mathrm{L}^{\prime}}, \\
& \dot{\mathrm{g}}^{\prime \prime}=-\frac{\partial \mathrm{F}^{\prime \prime}}{\partial \mathrm{G}^{\prime \prime}},
\end{aligned}
$$


and

$$
\dot{\mathrm{h}}^{\prime \prime}=-\frac{\partial \mathrm{F}^{\prime \prime}}{\partial \mathrm{H}^{\prime \prime}},
$$

where $F^{\prime \prime}=\mathcal{F}_{0}^{\prime \prime}+F_{1}^{\prime \prime}+F_{2}^{\prime \prime}$, was integrated by making use of a method involving elliptic integrals. This method has appeared in Reference 6, where it was applied to a different problem. In the previous version, it was assumed that $\mathrm{J}_{3}, \mathrm{~J}_{4}, \mathrm{~J}_{5}$, and all other spherical harmonics of the moon were of third order or smaller, making the method involving elliptic integrals applicable. Since these and other spherical harmonics of the moon are probably not that small (Reference 3 , for example), this method is no longer applicable. Thus it is suggested that the equations of motion for the secular Hamiltonian $\mathrm{F}^{\prime \prime}$ be integrated numerically. We have

$$
\mathrm{F}^{\prime \prime}=\mathrm{F}_{0}^{\prime \prime}+\mathrm{F}_{1}^{\prime \prime}+\mathrm{F}_{2}^{\prime \prime}+\mathrm{F}_{3}^{\prime \prime}
$$

where

$$
\begin{aligned}
& \mathrm{F}_{0}^{\prime \prime}-\frac{\mu^{2}}{2 \mathrm{~L}^{\prime 2}}, \\
& \mathrm{~F}_{1}^{\prime \prime}=\mathrm{n}_{\mathbb{C}}^{*} \mathrm{H}^{\prime \prime} .
\end{aligned}
$$

$\mathrm{F}_{2}$ " is given by Equation 78, and $\mathrm{F}_{3}$ " is given by Equation 132 . The system of equations to be integrated numerically is as follows:

$$
\begin{aligned}
& \dot{\mathrm{G}}^{\prime \prime}=\frac{\partial \mathrm{F}^{\prime \prime}}{\partial \mathrm{g}^{\prime \prime}}=\frac{\partial \mathrm{F}_{2}^{\prime \prime}}{\partial \mathrm{g}^{\prime \prime}}+\frac{\partial \mathrm{F}_{3}^{\prime \prime}}{\partial \mathrm{g}^{\prime \prime}}, \\
& \dot{i}=-\frac{\partial \mathrm{F}^{\prime \prime}}{\partial \mathrm{L}^{\prime \prime}}=\mathrm{n}^{\prime}-\frac{\partial \mathrm{F}_{2}^{\prime \prime}}{\partial \mathrm{L}^{\prime \prime}}-\frac{\partial \mathrm{F}_{3}^{\prime \prime}}{\partial \mathrm{L}^{\prime \prime}} . \\
& \dot{\mathrm{g}}^{\prime \prime}=-\frac{\partial \mathrm{F}^{\prime \prime}}{\partial \mathrm{G}^{\prime \prime}}=-\frac{\partial \mathrm{F}_{2}^{\prime \prime}}{\partial \mathrm{G}^{\prime \prime}}-\frac{\partial \mathrm{F}_{3}^{\prime \prime}}{\partial \mathrm{G}^{\prime \prime}},
\end{aligned}
$$

and

$$
\dot{\mathrm{h}}^{\prime \prime}=-\frac{\partial \mathrm{F}^{\prime \prime}}{\partial \mathrm{H}^{\prime \prime}}=-\mathrm{n}_{\mathbb{C}}^{*}-\frac{\partial \mathrm{F}_{2}^{\prime \prime}}{\partial \mathrm{H}^{\prime \prime}}-\frac{\partial \mathrm{F}_{3}^{\prime \prime}}{\partial \mathrm{H}^{\prime \prime}} \cdot
$$


Again, it is more convenient to compute the partials with respect to Keplerian elements, and use the expressions

$$
\begin{aligned}
& \frac{\partial F^{\prime \prime}}{\partial L^{\prime \prime}}=2 \sqrt{\frac{a^{\prime \prime}}{\mu}} \frac{\partial F^{\prime \prime}}{\partial a^{\prime \prime}}+\frac{1-e^{\prime \prime 2}}{e^{n} \sqrt{\mu a^{n}}} \frac{\partial F^{\prime \prime}}{\partial e^{\prime \prime}}, \\
& \frac{\partial \mathrm{F}^{\prime \prime}}{\partial \mathrm{G}^{\prime \prime}}=-\frac{1}{\mathrm{e}^{\prime \prime}} \sqrt{\frac{1-\mathrm{e}^{\prime \prime 2}}{\mu \mathrm{a}^{\prime \prime}}} \frac{\partial \mathrm{F}^{\prime \prime}}{\partial \mathrm{e}^{\prime \prime}}+\sqrt{\mu \mathrm{a}^{\prime \prime}} \frac{\cot \mathrm{I}^{\prime \prime}}{\overline{\left.\mathrm{e}^{\prime \prime 2}\right)}} \frac{\partial \mathrm{F}^{\prime \prime}}{\partial \mathrm{I}^{\prime \prime}},
\end{aligned}
$$

and

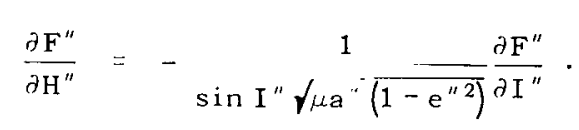

Thus,

$$
\begin{aligned}
& \frac{\partial \mathrm{F}_{2}^{\prime \prime}}{\partial \mathrm{a}^{\prime \prime}}+\frac{\partial \mathrm{F}_{3}^{\prime \prime}}{\partial \mathrm{a}^{\prime \prime}}=\frac{1}{8 \times}\left(\frac{\mathrm{n}_{\overparen{C}}}{\mathrm{n}^{\prime}}\right)^{2} \mathrm{n}^{\prime} \frac{\sqrt{\mu}}{\mathrm{a}^{\prime \prime}}\left[-\left(2+3 \mathrm{e}^{\prime 2}\right)\left(1-3 \cos ^{2} \mathrm{I}^{\prime \prime}\right)+15 \mathrm{e}^{\prime \prime 2} \sin ^{2} \mathrm{I}^{\prime \prime} \cos 2 \mathrm{~g}^{\prime \prime}\right]
\end{aligned}
$$

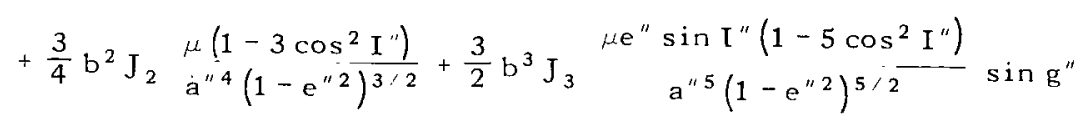

$$
\begin{aligned}
& +\frac{15}{128} b^{4} J_{4} a^{\prime \prime} 6\left(1-e^{\mu / 2}\right)^{7 / 2}\left[\left(2+3 e^{\prime \prime 2}\right)\left(3-30 \cos ^{2} I^{\prime \prime}+35 \cos ^{4} I^{\prime \prime}\right)\right.
\end{aligned}
$$

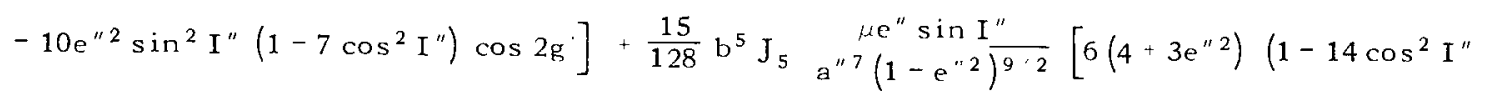

$$
\begin{aligned}
& \left.\left.+21 \cos ^{4} I^{\prime \prime}\right) \sin g^{\prime \prime}-7 e^{\prime 2} \sin ^{2} I^{\prime \prime}\left(1-9 \cos ^{2} I^{\prime \prime}\right) \sin 3 g^{\prime \prime}\right] \\
& +\frac{63}{256 \epsilon^{2}}\left(\frac{n_{\mathbb{C}}}{n_{\mathbb{C}}^{*}}\right)\left(\frac{n_{\mathbb{C}}}{n^{\prime}}\right)^{3} \mathrm{n}^{\prime 2} \mathrm{a}^{\prime \prime}\left(1-\mathrm{e}^{\prime \prime 2}\right)^{1 / 2} \cos \mathrm{I}^{\prime \prime}\left[\left(2+33 \mathrm{e}^{\prime \prime 2}\right)-\left(2-17 \mathrm{e}^{\prime \prime}\right) \cos ^{2} \mathrm{I}^{\prime \prime}+15 \mathrm{e}^{\prime 2} \sin ^{2} \mathrm{I}^{\prime \prime} \cos 2 \mathrm{~g}^{\prime \prime}\right] \\
& -\frac{27}{64 \epsilon}\left(\frac{n_{\mathbb{C}}}{n_{\mathbb{C}}^{*}}\right)\left(\frac{n_{\mathbb{C}}}{n^{\prime}}\right) b^{2} J_{22} \frac{n^{\prime 2} \sin ^{2} I^{\prime \prime} \cos I^{\prime \prime}}{a^{\prime \prime}\left(1-e^{\prime \prime 2}\right)^{2}}\left[2\left(2+3 e^{\prime \prime 2}\right)+15 e^{\prime 2} \cos 2 g^{\prime \prime}\right] \\
& -\frac{117}{8}\left(\frac{n^{\prime}}{n_{C}^{*}}\right) b^{4} J_{22}^{2} n^{\prime 2} a^{\prime \prime} \frac{\sin ^{2} I^{\prime \prime} \cos I^{\prime \prime}}{\left(1-e^{\prime \prime 2}\right)^{7 / 2}}+\frac{1}{8} n_{\oplus}^{2} a^{\prime \prime}\left[-\left(2+3 e^{\prime \prime 2}\right)\left(1-3 \cos ^{2} I^{\prime \prime}\right)+15 e^{\prime \prime 2} \sin ^{2} I^{\prime \prime} \cos 2 g^{\prime \prime}\right],
\end{aligned}
$$



$-\frac{15}{256} b^{5} J_{5} \frac{\mu e^{\prime \prime} \cos I^{\prime \prime}}{a^{\prime \prime}\left(1-e^{\prime \prime 2}\right)^{9 / 2}}\left[2\left(4+3 e^{\prime 2}\right)\left(29-126 \cos ^{2} I^{\prime \prime}+105 \cos ^{4} I^{\prime \prime}\right) \sin g^{\prime \prime}\right.$

$$
\begin{aligned}
& \left.-7 e^{\prime \prime 2} \sin ^{2} I^{\prime \prime}\left(7-15 \cos ^{2} I^{\prime \prime}\right) \sin 3 g^{\prime \prime}\right] \\
& -\frac{9}{128 \epsilon^{2}}\left(\frac{n_{\overparen{C}}}{n_{\overparen{C}}^{*}}\right)\left(\frac{n_{\overparen{C}}}{n^{\prime}}\right)^{3} n^{\prime 2} a^{\prime \prime 2}\left(1-e^{\prime \prime 2}\right)^{1 / 2} \sin I^{\prime \prime}\left[2+33 e^{\prime 2}-3\left(2-17 e^{\prime 2}\right) \cos ^{2} I^{\prime \prime}\right. \\
& \left.+15 e^{\prime \prime}\left(1-3 \cos ^{2} \mathrm{I}^{\prime \prime}\right) \cos 2 \mathrm{~g}^{\prime \prime}\right] \\
& -\frac{9}{32 \epsilon}\left(\frac{n_{\mathbb{C}}}{n_{\mathbb{C}}^{*}}\right)\left(\frac{n_{\mathbb{C}}}{n^{\prime}}\right) b^{2} J_{22} \stackrel{n^{\prime 2} \sin I^{\prime \prime}\left(1-3 \cos ^{2} I^{\prime \prime}\right)}{\left(1-e^{\prime \prime 2}\right)^{2}}\left[2\left(2+3 e^{\prime \prime 2}\right)+15 e^{\prime \prime 2} \cos 2 g^{\prime \prime}\right]
\end{aligned}
$$

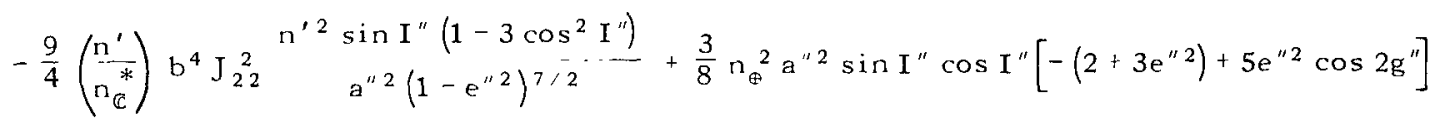

$$
\begin{aligned}
& +\frac{3}{4 \epsilon} j_{2} R_{\circledast}^{2} n_{C}^{2}\left(1-3 \cos ^{2} I_{\oplus}\right) \sin I^{\prime \prime} \cos I^{\prime \prime}\left\{1-\beta^{\prime \prime 2}\left[1+2\left(1-e^{\prime \prime 2}\right)^{1 / 2}\right] \cos 2 g^{\prime \prime}\right\}
\end{aligned}
$$

and

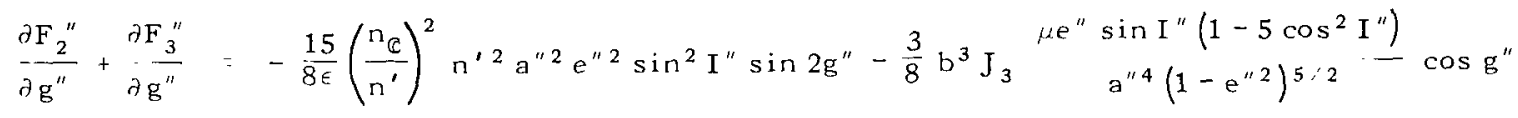

$$
\begin{aligned}
& -\frac{15}{32} b^{4} J_{4}-\frac{\mu e^{\prime 2}}{\sin ^{2} I^{\prime \prime}\left(1-7 \cos ^{2} I^{\prime \prime}\right)} \sin 2 g^{\prime \prime}
\end{aligned}
$$

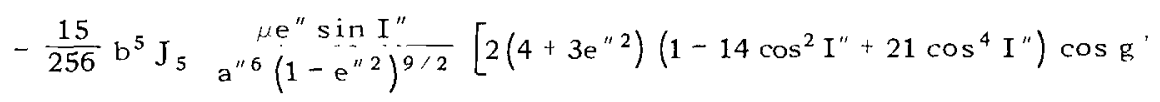

$$
\begin{aligned}
& \left.-7 e^{\prime 2} \sin ^{2} I^{\prime \prime}\left(1-9 \cos ^{2} I^{\prime \prime}\right) \cos 3 g^{\prime \prime}\right] \\
& -\frac{135}{64 \epsilon^{2}}\left(\frac{n_{\mathbb{C}}}{n_{\mathbb{C}}^{*}}\right)\left(\frac{n_{\mathbb{C}}}{n^{\prime}}\right)^{3} n^{\prime 2} a^{\prime \prime 2} e^{\prime \prime 2}\left(1-e^{\prime 2}\right)^{1 / 2} \sin ^{2} I^{\prime \prime} \cos I^{\prime \prime} \sin 2 g^{\prime \prime}
\end{aligned}
$$




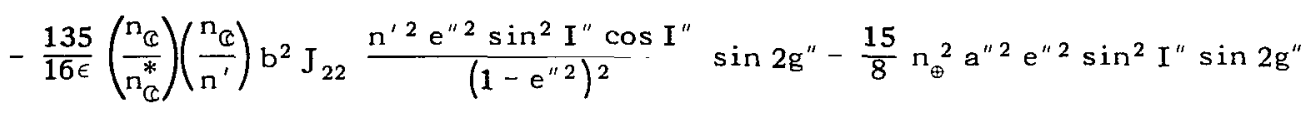

$$
\begin{aligned}
& +\frac{3^{l}}{4 \epsilon} j_{2} R_{\oplus}^{2} n_{\mathscr{C}}^{2}\left(1-3 \cos ^{2} I_{\oplus}\right) \beta^{\prime \prime}\left[1+2\left(1-e^{\prime \prime 2}\right)^{1 / 2}\right] \sin ^{2} I^{\prime \prime} \sin 2 g^{\prime \prime}
\end{aligned}
$$

\section{SUMMARY OF THE DEVELOPMENT}

The short-period terms are given by

$$
\begin{aligned}
& l=l^{\prime}-\frac{\partial \mathrm{S}_{2}}{\partial \mathrm{L}^{\prime}}=l^{\prime}+\Delta l, \\
& \mathrm{~g}=\mathrm{g}^{\prime}-\frac{\partial \mathrm{S}_{2}}{\partial \mathrm{G}^{\prime}}=\mathrm{g}^{\prime}+\Delta \mathrm{g}, \\
& \mathrm{h}=\mathrm{h}^{\prime}-\frac{\partial \mathrm{S}_{2}}{\partial \mathrm{H}^{\prime}}=\mathrm{h}^{\prime}+\Delta \mathrm{h}, \\
& \mathrm{L}=\mathrm{L}^{\prime}+\frac{\partial \mathrm{S}_{2}}{\partial l}=\mathrm{L}^{\prime}+\Delta \mathrm{L}, \\
& \mathrm{G}=\mathrm{G}^{\prime}+\frac{\partial \mathrm{S}_{2}}{\partial \mathrm{g}}=\mathrm{G}^{\prime}+\Delta \mathrm{G},
\end{aligned}
$$

and

$$
H=H^{\prime}+\frac{\partial S_{2}}{\partial h}=H^{\prime}+\Delta H .
$$

Long-period terms are obtained from

$$
\begin{aligned}
& l^{\prime}=l^{\prime \prime}-\frac{\partial \mathbf{S}_{1}^{\prime}}{\partial \mathrm{L}^{\prime \prime}}-\frac{\partial \mathbf{S}_{2}{ }^{\prime}}{\partial \mathrm{L}^{\prime \prime}}-\frac{\partial \mathbf{S}_{3}^{\prime}}{\partial \mathrm{L}^{\prime \prime}}=l^{\prime \prime}+\Delta l^{\prime}, \\
& \mathbf{g}^{\prime}=\mathbf{g}^{\prime \prime}-\frac{\partial \mathbf{S}_{1}^{\prime}}{\partial \mathrm{G}^{\prime \prime}}-\frac{\partial \mathbf{S}_{2}^{\prime}}{\partial \mathrm{G}^{\prime \prime}}-\frac{\partial \mathbf{S}_{3}^{\prime}}{\partial \mathrm{G}^{\prime}}=\mathbf{g}^{\prime}+\wedge \mathbf{g}^{\prime}, \\
& \mathbf{h}^{\prime}=\mathrm{h}^{\prime \prime}-\frac{\partial \mathrm{S}_{1}^{\prime}}{\partial \mathrm{H}^{\prime \prime}}-\frac{\partial \mathrm{S}_{2}^{\prime}}{\partial \mathrm{H}^{\prime}}-\frac{\partial \mathbf{S}_{3}^{\prime}}{\partial \mathrm{H}^{\prime}}=\mathrm{h}^{\prime \prime}+\Delta \mathrm{h}^{\prime}, \\
& \mathrm{L}^{\prime}=\mathrm{L}^{\prime \prime},
\end{aligned}
$$




$$
\mathbf{G}^{\prime}=\mathbf{G}^{\prime \prime}+\frac{\partial \mathbf{S}_{1}^{\prime}}{\partial \mathrm{g}^{\prime}}+\frac{\partial \mathbf{S}_{2}^{\prime}}{\partial \mathrm{g}^{\prime}}+\frac{\partial \mathbf{S}_{3}^{\prime}}{\partial \mathrm{g}^{\prime}}=\mathbf{G}^{\prime \prime}+\Delta \mathbf{G}^{\prime},
$$

and

$$
H^{\prime}=H^{\prime \prime}+\frac{\partial S_{1}^{\prime}}{\partial h^{\prime}}+\frac{\partial S_{2}^{\prime}}{\partial h^{\prime}}+\frac{\partial S_{3}^{\prime}}{\partial h^{\prime}}=H^{\prime \prime}+\Delta H^{\prime}
$$

Secular perturbations and perturbations depending only on $g^{\prime \prime}$ result from the integration of Equations 180:

$$
\begin{aligned}
& \Delta l^{\prime \prime}=\int\left(\mathrm{n}^{\prime}-\frac{\partial \mathrm{F}_{2}{ }^{\prime \prime}}{\partial \mathrm{L}^{\prime \prime}}-\frac{\partial \mathrm{F}_{3}{ }^{\prime \prime}}{\partial \mathrm{L}^{\prime \prime}}\right) \mathrm{dt}=l^{\prime \prime}-l_{0}^{\prime \prime}, \\
& \Delta \mathrm{g}^{\prime \prime}=-\int\left(\frac{\partial \mathrm{F}_{2}^{\prime \prime}}{\partial \mathrm{G}^{\prime \prime}}+\frac{\partial \mathrm{F}_{3}{ }^{\prime \prime}}{\partial \mathrm{G}^{\prime \prime}}\right) \mathrm{dt}=\mathrm{g}^{\prime \prime}-\mathrm{g}_{0}^{\prime \prime}, \\
& \Delta \mathrm{h}^{\prime \prime}=-\int\left(\mathrm{n}_{\mathbb{C}}^{*}+\frac{\partial \mathrm{F}_{2}{ }^{\prime \prime}}{\partial \mathrm{H}^{\prime \prime}}+\frac{\partial \mathrm{F}_{3}{ }^{\prime \prime}}{\partial \mathrm{H}^{\prime \prime}}\right) \mathrm{dt}=\mathrm{h}^{\prime \prime}-\mathrm{h}_{0}^{\prime \prime}, \\
& \Delta \mathrm{L}^{\prime \prime}=0, \\
& \Delta \mathrm{G}^{\prime \prime}=\int\left(\frac{\partial \mathrm{F}_{2}^{\prime \prime}}{\partial \mathrm{g}^{\prime \prime}}+\frac{\partial \mathrm{F}_{3}{ }^{\prime \prime}}{\partial \mathrm{g}^{\prime \prime}}\right) \mathrm{dt}=\mathrm{G}^{\prime \prime}-\mathrm{G}_{0}{ }^{\prime \prime},
\end{aligned}
$$

and

$$
\Delta \mathrm{H}^{\prime \prime}=0 \text {. }
$$

Then, the perturbations in the Keplerian elements a, e, and I are given by

$$
\mathbf{a}=\mathbf{a}^{\prime}+2 \mathbf{a}^{\prime} \frac{\Delta \mathrm{L}}{\mathrm{L}^{\prime}}
$$

and

$$
\mathbf{a}^{\prime}=\mathbf{a}^{\prime \prime}=\frac{\mathrm{L}^{\prime \prime 2}}{\mu},
$$




$$
\begin{aligned}
& \mathrm{e}=\mathrm{e}^{\prime}+\frac{1-\mathrm{e}^{\prime 2}}{\mathrm{e}^{\prime}}\left(\frac{\Delta \mathrm{L}}{\mathrm{L}^{\prime}}-\frac{\Delta \mathrm{G}}{\mathrm{G}^{\prime}}\right), \\
& \mathrm{e}^{\prime}=\mathrm{e}^{\prime \prime}-\frac{1-\mathrm{e}^{\prime \prime} 2}{\mathrm{e}^{\prime \prime}} \frac{\Delta \mathrm{G}^{\prime}}{\mathrm{G}^{\prime \prime}}-\frac{1-\mathrm{e}^{\prime \prime}}{2 \mathrm{e}^{\prime \prime}}\left(\frac{\Delta \mathrm{G}^{\prime}}{\mathrm{G}^{\prime \prime}}\right)^{2}, \\
& \mathrm{e}^{\prime \prime}=\mathrm{e}_{0}^{\prime \prime}-\frac{1-\mathrm{e}_{0}^{\prime \prime}}{\mathrm{e}_{0}^{\prime \prime}} \frac{\Delta \mathrm{G}^{\prime \prime}}{\mathrm{G}_{0}^{\prime \prime}},
\end{aligned}
$$

and

$$
\mathrm{e}_{0}^{\prime \prime}=1-\frac{\mathrm{G}_{0}{ }^{\prime 2}}{\mathrm{~L}^{\prime \prime 2}}
$$

$I=I^{\prime}+\cot I^{\prime}\left(\frac{\Delta G}{G^{\prime}}-\frac{\Delta H}{H^{\prime}}\right)$

$I^{\prime}=I^{\prime \prime}+\cot I^{\prime \prime}\left(\frac{\Delta G^{\prime}}{G^{\prime \prime}}-\frac{\Delta H^{\prime}}{H^{\prime \prime}}\right)+\frac{\cos I^{\prime \prime}}{\sin ^{3} I^{\prime \prime}}\left(\frac{\Delta G^{\prime}}{G^{\prime \prime}}\right)\left(\frac{\Delta H^{\prime}}{H^{\prime \prime}}\right)-\frac{1}{2} \frac{\cos I^{\prime \prime}\left(1+\sin ^{2} \mathrm{I}^{\prime \prime}\right)}{\sin ^{3} I^{\prime \prime}}\left(\frac{\Delta G^{\prime}}{G^{\prime \prime}}\right)^{2}-\frac{1}{2} \cot ^{3} I^{\prime \prime}\left(\frac{\Delta H^{\prime}}{H^{\prime \prime}}\right)^{2}$,

$I^{\prime \prime}=I_{0}^{\prime \prime}+\cot I_{0} " \frac{\Delta G^{\prime \prime}}{G_{0}^{\prime \prime}}$,

and

$\mathrm{I}_{0}^{\prime \prime}=\cos ^{-1}\left(\frac{\mathrm{H}^{\prime \prime}}{\mathrm{G}_{0}^{\prime \prime}}\right)$.

POSITION AND VELOCITY: $e \neq 0 ; 1 \neq 0^{\circ}, 180^{\circ}$

From the elements L, G, H, l, g, h, one can obtain the coordinates and the components of velocity as follows.

Obtain a, e, and I from the following equations:

$$
\begin{aligned}
& a=\frac{\mathrm{L}^{2}}{\mu}, \\
& \mathrm{e}=\sqrt{1-\mathrm{G}^{2} / \mathrm{L}^{2}},
\end{aligned}
$$

and

$$
I=\arccos \frac{\mathrm{H}}{\mathrm{G}} \quad\left(0^{\circ}<\mathrm{I}<180^{\circ}\right) .
$$


Now solve Kepler's equation

$$
E-e \sin E=l
$$

to obtain E.

Then compute $\mathrm{r}$ from

$$
r=a(1-e \cos E) \text {. }
$$

Next obtain f from

$$
\cos f=\frac{a}{r}(\cos E-e)
$$

and

$$
\sin \mathrm{f}=\frac{\mathrm{a}}{\mathrm{r}} \frac{\mathrm{G}}{\mathrm{L}} \sin \mathrm{E} .
$$

Now compute

$$
\begin{aligned}
& A_{x}=a(\cos g \cos h-\sin g \sinh \cos I), \\
& B_{x}=a \frac{G}{L}(-\sin g \cosh -\cos g \sinh \cos I), \\
& A_{y}=a(\cos g \sinh +\sin g \cos h \cos I), \\
& B_{y}=a \frac{G}{L}(-\sin g \sinh +\cos g \cos h \cos I), \\
& A_{z}=a \sin g \sin I,
\end{aligned}
$$

and

$$
B_{z}=a \frac{G}{L} \cos g \sin I .
$$

Then,

$$
\left(\begin{array}{l}
x \\
y \\
z
\end{array}\right)=\left(\begin{array}{ccc}
A_{x} & B_{x} & 0 \\
A_{y} & B_{y} & 0 \\
A_{z} & B_{z} & 0
\end{array}\right)\left(\begin{array}{c}
\cos E-e \\
\sin E^{\cdot} \\
0
\end{array}\right) .
$$


Since the system is rotating,

$$
\begin{aligned}
& \dot{A}_{x}=n_{C}^{*} A_{y}, \\
& \dot{B}_{x}=n_{C}^{*} B_{y}, \\
& \dot{A}_{y}=-n_{C}^{*} A_{x},
\end{aligned}
$$

and

$$
\dot{\mathrm{B}}_{\mathrm{y}}=-\mathrm{n}_{\mathrm{C}}^{*} \mathrm{~B}_{\mathrm{x}} .
$$

Therefore,

$$
\begin{aligned}
\left(\begin{array}{c}
\dot{x} \\
\dot{y} \\
\dot{z}
\end{array}\right) & =n_{\mathbb{C}}^{*}\left(\begin{array}{ccc}
A_{y} & B_{y} & 0 \\
-A_{x} & -B_{x} & 0 \\
0 & 0 & 0
\end{array}\right)\left(\begin{array}{c}
\cos E-e \\
\sin E \\
0
\end{array}\right) \\
& +\frac{a}{r} n\left(\begin{array}{ccc}
A_{x} & B_{x} & 0 \\
A_{y} & B_{y} & 0 \\
A_{z} & B_{z} & 0
\end{array}\right)\left(\begin{array}{c}
-\sin E \\
\cos E \\
0
\end{array}\right) .
\end{aligned}
$$

\section{ACKHOWLEDGMENTS}

The authors would like to thank Messrs. E. M. Standish, Jr. (Yale University), and C. E. Velez (Goddard Space Flight Center) for their help in checking some of the mathematics and in programming the theory.

\footnotetext{
Goddard Space Flight Center

National Aeronautics and Space Administration

Greenbelt, Maryland, July 22, 1968

$311-07-21-01-51$
}

\section{REFERENCES}

1. Akim, E. L., "Determination of the Gravitational Field of the Moon by the Motion of AMS Luna-10," Doklady Akademii Nauk SSSR, Mekhanika, 170(4), 799-802, 1966.

2. Lorell, J., and Sjogren, W. L., "Space Programs Summary No. 37-46," Jet Propulsion Laboratory, Pasadena, Calif., Vol. IV, p. 1, August 31, 1967. 
3. Tolson, R. H., and Gapcynski, J. P., "An Analysis of the Lunar Gravitational Field as Obtained from Lunar Orbiter Tracking Data," presented at the IQSY/COSPAR Assemblies, London, England, July 17-28, 1967.

4. Plummer, H. C., "An Introductory Treatise on Dynamical Astronomy," New York: Dover Publications, 1960.

5. Giacaglia, G., Murphy, J., and Felsentreger, T., "The Motion of a Satellite of the Moon," NASA Technical Memorandum X-55295, November 1965.

6. Kozai, Y., Astronomical Journal 67, 591-598, 1962.

\section{BIBLIOGRAPHY}

Brouwer, D., Astronomical Journal 64, 378-397, 1959.

Brouwer, D., and Clemence, G., "Methods of Celestial Mechanics," New York: Academic Press, 1961.

Jeffreys, Sir Harold, Proc. Roy. Soc. London, Ser. A, No. 1446, 296, 245-247, 1967.

Kozai, Y., Journal of the Astronomical Society of Japan, 15, 301-312, 1963.

Moulton, F. R., "An Introduction to Celestial Mechanics," New York: The Macmillan Company, 1914. 


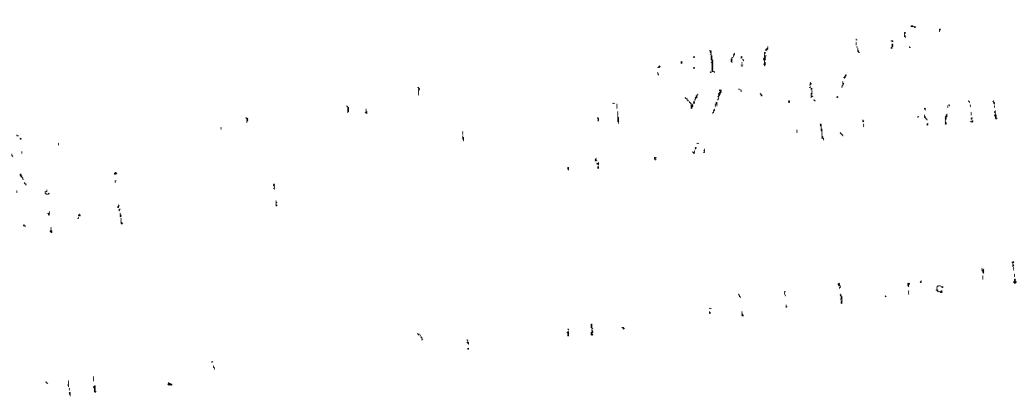

"The aeronautical and space activities of the United States shall be conducted so as to contribute... to the expansion of buman knowledge of phenomena in the atmosphere and space. The Administration shall provide for the widest practicable and appropriate dissemination of information concerning its activities and the results thereof."

\section{- National Aeronautics and Space ACt of 1958}

\section{NASA SCIENTIFIC AND TECHNICAL PUBLICATIONS}

TECHNICAL REPORTS: Scientific and technical information considered important, complete, and a lasting contribution to existing knowledge.

TECHNICAL NOTES: Information less broad in scope but nevertheless of importance as a contribution to existing knowledge.

\section{TECHNICAL MEMORANDUMS:}

Information receiving limited distribution because of preliminary data, security classification, or other reasons.

CONTRACTOR REPORTS: Scientific and technical information generated under a NASA contract or grant and considered an important contribution to existing knowledge.
TECHNICAL TRANSLATIONS: Information published in a foreign language considered to merit NASA distribution in English.

SPECIAL PUBLICATIONS: Information derived from or of value to NASA activities. Publications include conference proceedings, monographs, data compilations, handbooks, sourcebooks, and special bibliographies.

\section{TECHNOLOGY UTILIZATION}

PUBLICATIONS: Information on technology used by NASA that may be of particular interest in commercial and other non-aerospace applications. Publications include Tech Briefs, Technology Utilization Reports and Notes, and Technology Surveys.

Details on the availability of these publications may be obtained from:

SCIENTIFIC AND TECHNICAL INFORMATION DIVISION

NATIONAL AERONAUTICS AND SPACE ADMINISTRATION

Washington, D.C. 20546 